\title{
On the Behaviour of Singular Semigroups in Intermediate and Interpolation Spaces and Its Applications to Maximal Regularity for Degenerate Integro-Differential Evolution Equations
}

\author{
Alberto Favaron and Angelo Favini \\ Dipartimento di Matematica, Università degli Studi di Bologna, Piazza di Porta San Donato 5, 40126 Bologna, Italy \\ Correspondence should be addressed to Angelo Favini; angelo.favini@unibo.it
}

Received 6 May 2013; Accepted 25 June 2013

Academic Editor: Rodrigo Lopez Pouso

Copyright (C) 2013 A. Favaron and A. Favini. This is an open access article distributed under the Creative Commons Attribution License, which permits unrestricted use, distribution, and reproduction in any medium, provided the original work is properly cited.

For those semigroups, which may have power type singularities and whose generators are abstract multivalued linear operators, we characterize the behaviour with respect to a certain set of intermediate and interpolation spaces. The obtained results are then applied to provide maximal time regularity for the solutions to a wide class of degenerate integro- and non-integro-differential evolution equations in Banach spaces.

\section{Introduction}

Let $X$ be a complex Banach space and let $\left\{\mathscr{T}_{A}(t)\right\}_{t \geq 0}$ be a semigroup of operators on $X$, which is generated by a multivalued linear operator $A: \mathscr{D}(A) \subseteq X \rightarrow X$ and which may have a power type singularity at the origin $t=0$, that is,

$$
\begin{gathered}
\left\|\mathscr{T}_{A}(t)\right\|_{\mathscr{L}(X)} \leq C_{0} t^{v}, \quad \forall t>0, \\
\mathscr{T}_{A}(0) x=x, \quad \forall x \in X,
\end{gathered}
$$

for some nonnegative constant $C_{0}$ and nonpositive exponent $\nu$, where $\mathscr{L}(X)$ denotes the Banach algebra of all endomorphisms of $X$ endowed with the uniform operator norm. In this context our aim here is twofold. The first is to characterize the behaviour of $\left\{\mathscr{T}_{A}(t)\right\}_{t \geq 0}$ with respect to some intermediate and interpolation spaces between $X$ and the domain $\mathscr{D}(A)$ of $A$. The second is to investigate how this behaviour reflects on the question of maximal time regularity for the solutions to a class of degenerate integro- and non-integrodifferential initial value problems in $X$.

The class of operators we will deal with consists precisely of those multivalued linear operators $A$ whose single-valued resolvents satisfy the following estimate:

$$
\left\|(\lambda I-A)^{-1}\right\|_{\mathscr{L}(X)} \leq C(|\lambda|+1)^{-\beta}, \quad \forall \lambda \in \Sigma_{\alpha} .
$$

Here, $I$ is the identity operator, $C$ is a positive constant, $\beta \epsilon$ $(0,1]$, and $\Sigma_{\alpha}$ is the complex region $\{z \in \mathbf{C}: \mathfrak{R e} z \geq$ $\left.-c(|\Im \mathrm{m} z|+1)^{\alpha}, \Im \mathrm{m} z \in \mathbf{R}\right\}, c>0, \alpha \in[\beta, 1]$. It thus happens (cf. [1-3]) that $A$ is the infinitesimal generator of a semigroup of linear bounded operators in $X$ satisfying (1) with $\nu=v_{\alpha, \beta}$, where $v_{\alpha, \beta}=(\beta-1) / \alpha$.

To outline the motivations of our research, let us assume for a moment that $A$ is a single-valued linear operator satisfying (2). It is well known that if $\beta=1$, then $A$ is the infinitesimal generator of a bounded analytic semigroup. For this case, an extensive literature exists concerning the behaviour of $\left\{\mathscr{T}_{A}(t)\right\}_{t \geq 0}$ with respect to the real interpolation spaces $(X, \mathscr{D}(A))_{\gamma, p}, \gamma \in(0,1), p \in[1, \infty]$, and its application to questions of maximal regularity for the solutions to nondegenerate (possibly nonautonomous) integro- and non-integrodifferential abstract Cauchy problems. See, for instance, [4-11]. Due to (1) with $v=v_{1, \beta}$, the case of $\alpha=$ 1 and $\beta \in(0,1)$ is definitely worsened and the literature for it is considerably less conspicuous, although estimate of type (2), with $\left(\Re \mathrm{e} \lambda+|\Im \mathrm{m} \lambda|^{\beta}\right)^{-1}$ in place of $(|\lambda|+1)^{-\beta}$, goes back even to [12, Remark p. 383] in the ambit of Abel summable semigroups admitting uniform derivatives of all orders. One of the main problems with the case $\beta \in(0,1)$ is that some equivalent characterizations of $(X, \mathscr{D}(A))_{\gamma, p}$ begin to fail (cf. [13]), so that some spaces which were just real 
interpolation spaces between $X$ and $\mathscr{D}(A)$ in the case $\beta=$ 1 become only intermediate spaces in the case $\beta \in(0,1)$. However, avoiding questions of interpolation theory and of maximal regularity, a quite satisfactorily semigroup theory for the single-valued case with $\beta \in(0,1)$ and its application to the unique solvability of some concrete partial (nonintegro-) differential equations have been developed in [1418]. Since the multivalued case embraces the single-valued one, our contribution in this field is to fill this gap, supplying a theory for the behaviour of singular semig $n$ intermediate and interpolation spaces which, in the case $\beta=1$, reduces to that in $[9,11]$. As an effect of this theory, there is the possibility of investigating questions of maximal time regularity for an entire class of nondegenerate evolution equations which does not fall within the case $\beta=1$.

The case when $A$ is really a multivalued linear operator arises naturally when we shift our attention to degenerate evolution equations of the type considered in [1-3]. There, a semigroup theory for multivalued linear operators was introduced as a tool to handle degenerate equations by means of analogous techniques of the nondegenerate ones. Such a theory has been then successfully applied to questions of maximal regularity for the solutions to a wide class of degenerate integro- and non-integrodifferential equations. We quote [2, 19-23] where, in general and unless $\beta=1$, it is shown that the time regularity of the solutions decreases with respect to that of the data. In this respect, we mention the recent results in [20] where, under an additional condition of space regularity on the data and provided that $\alpha$ and $\beta$ are large enough, the loss of time regularity is restored. Regrettably (cf. the appendix below), we have found some inaccuracies in [20, Section 4], and for this reason we must indicate some changes to that paper. On the other side, fortunately, the basic idea in [20] is correct and remedy can be applied to all the inappropriate items. Furthermore, unexpectedly, we will see that the more delicate approach followed in this paper not only corrects the mistakes in [20], but also gives rise to an effective improvement of the achievable results. In fact, here, we will straighten out, refine, and extend [20], enlarging the class of the admissible spaces to which the data may belong, weakening the assumption for the pair $(\alpha, \beta)$, and complicating the structure of the underlying equations. This is why we will first analyze the behaviour of the semigroup generated by $A$ with respect to some intermediate and interpolation spaces which turn out to be equivalent only in the case $\beta=1$. Indeed, the phenomena exhibited in [13] for the single-valued case extend to the multivalued one (cf. [24]), and, until now, for the mentioned behaviour there exist no more than some partial results obtained in $[2,19,24]$.

We now give the detailed plan of the paper. In Section 2, for a multivalued linear operator $A$ having domain $\mathscr{D}(A)$ and satisfying (2), we introduce the corresponding generated semigroup $\left\{\mathrm{e}^{t A}\right\}_{t \geq 0}$. This leads us to define also the linear bounded operators $\left[(-A)^{\theta}\right]^{\circ} \mathrm{e}^{t A}, \mathfrak{R e} \theta \geq 0, t>0,\left(\left[(-A)^{0}\right]^{\circ}\right.$ $\left.e^{t A}=e^{t A}\right)$ and to recall the fundamental estimates for their $\mathscr{L}(X)$-norm. For the operators $\left[(-A)^{\theta}\right]^{\circ} \mathrm{e}^{t A}$ a semigroup type property is proven in Proposition 1 . We then introduce the spaces we will deal with in this paper, that is, the interpolation spaces $(X, \mathscr{D}(A))_{\gamma, p}$ and the spaces $X_{A}^{\gamma, P}$, $\gamma \in(0,1), p \in[1, \infty]$. Special attention is given to the embeddings linking these two classes of spaces which, in general, are equivalent only in the case $\beta=$ 1. Some relations existing between the spaces $X_{A}^{\gamma, p}$ for different values of $\gamma$ and $p$ are proven in Proposition 2 and discussed in Remarks 3-5. We conclude the section recalling the estimates proven in $[19,24]$ for the norms $\left\|\left[(-A)^{\theta}\right]^{\circ} \mathrm{e}^{t A}\right\|_{\mathscr{L}\left(X ;(X, \mathscr{D}(A))_{\gamma, p}\right)}, \mathfrak{R e} \theta \geq 0$, and $\|\left[(-A)^{1}\right]^{\circ}$ $\mathrm{e}^{t A} \|_{\mathscr{L}\left(Y_{\gamma}^{p} ; X\right)}, Y_{\gamma}^{p} \in\left\{(X, \mathscr{D}(A))_{\gamma, p}, X_{A}^{\gamma, p}\right\}$. In Remarks 7 and 8 we explain why, unless we renounce to optimality, in the case $\beta<$ 1 these estimates can not be directly extended to the norms $\left\|\left[(-A)^{\theta}\right]^{\circ} \mathrm{e}^{t A}\right\|_{\mathscr{L}\left(X ; X_{A}^{\gamma, p}\right)}$ and $\left\|\left[(-A)^{\theta}\right]^{\circ} \mathrm{e}^{t A}\right\|_{\mathscr{L}\left(Y_{\gamma}^{p} ; X\right)}, \mathfrak{R e} \theta \geq 1$, respectively.

In Section 3, we investigate the behaviour of the operators $\left[(-A)^{\theta}\right]^{\circ} \mathrm{e}^{t A}$ with respect to both of the spaces $(X, \mathscr{D}(A))_{\gamma, p}$ and $X_{A}^{\gamma, p}$. First, in Proposition 9, we deal with the norms $\left\|\left[(-A)^{\theta}\right]^{0} \mathrm{e}^{t A}\right\|_{\mathscr{L}\left(X ; X_{A}^{\gamma, p}\right)}, \mathfrak{R e} \theta \geq 0$, and we show that, except for replacing $(X, \mathscr{D}(A))_{\gamma, p}$ with $X_{A}^{\gamma, \infty}$ if $p=\infty$ and with $X_{A}^{\beta \gamma, p}$ if $p \in[1, \infty)$, the same estimates of [19] for the norms $\left\|\left[(-A)^{\theta}\right]^{\circ} \mathrm{e}^{t A}\right\|_{\mathscr{L}\left(X ;(X, \mathscr{D}(A))_{\gamma, p}\right)}$ continue to hold. The second significant result is Proposition 12 where, extending those in [24] to values of $\theta$ other than one, we establish estimates for the norms $\left\|\left[(-A)^{\theta}\right]^{0} \mathrm{e}^{t A}\right\|_{\mathscr{L}\left(Y_{\gamma}^{p} ; X\right)}, \mathfrak{R e} \theta \geq 1, Y_{\gamma}^{p} \in\left\{(X, \mathscr{D}(A))_{\gamma, p}\right.$, $\left.X_{A}^{\gamma, P}\right\}$. As a byproduct we deduce the basic Corollary 14 , which in Section 5 will be a key tool in proving the equivalence between the following problem (3) and the fixed-point equation (179). The estimates in Proposition 12 are then merged together with those in [19] to achieve estimates for the norms $\left\|\left[(-A)^{\theta}\right]^{0} \mathrm{e}^{t A}\right\|_{\mathscr{L}\left((X, \mathscr{D}(A))_{\gamma, p},(X, \mathscr{D}(A))_{\delta, p}\right)}, \mathfrak{R e} \theta \geq 1$. In particular, two different estimates are obtained, if $\gamma+\delta<1$ or not. For if $\gamma+\delta<1$, then (cf. the proof of Proposition 16) we can take advantage of the reiteration theorem for interpolation spaces and obtain estimates that, unless $\beta=1$, are better than those rougher estimates derived in the general case $\gamma, \delta \in(0,1)$ (see Remarks 17 and 18). We stress that if $\beta=1, \theta \in \mathbf{N}$ and $A$ is single-valued, then we restore the estimates in [9]. Finally, in Proposition 20, a combination of Propositions 9 and 12 yields the estimate for the norms $\left\|\left[(-A)^{\theta}\right]^{0} e^{t A}\right\|_{\mathscr{L}\left(X_{A}^{\gamma, p}, X_{A}^{\delta, p}\right)}$, $\mathfrak{R e} \theta \geq 1$. Since $\beta<1$, the spaces $X_{A}^{\sigma, q}$ are, in general, only intermediate spaces between $X$ and $\mathscr{D}(A)$ for $\sigma \in(0, \beta)$; here the reiteration theorem does not apply and a weaker result is obtained (cf. (101)-(103)).

The estimates of Section 3 are applied in Section 4 to study the time regularity of those operator functions $Q_{j}, j=$ $1, \ldots, 6$, that we will need in Section 5. In particular (cf. formula (106)), we modify the definition of $Q_{2}$ in $[20$, Section $4]$ in order that it is well defined, at least when acting on functions $g \in C^{\delta}([0, T] ; X), \delta \in((2-\alpha-\beta) / \alpha, 1)$ (cf. Corollary 26). Consequently, operators $Q_{3}$ and $Q_{4}$ in [20] change too, and the new $Q_{5}$ and $Q_{6}$ should be introduced (cf. formulae (107)-(110)). The Hölder in time regularity of the $Q_{j}$ 's is characterized in Lemmas $22,24,30$, and 32 and Propositions 29 and 36 . The main feature of these results is to show that the loss of regularity produced by $Q_{2}$ and 
$Q_{5}$ can be restored, in $Q_{3}$ and $Q_{6}$ respectively, employing the regularization property established in [20, Section 3] for a wide range of general convolution operators.

In Section 5 we analyze the maximal time regularity of the strict solutions $v$ to the following class of degenerate integrodifferential equations in a complex Banach space $X$ :

$$
\begin{gathered}
\frac{\mathrm{d}}{\mathrm{d} t}(M v(t))=\left[\lambda_{0} M+L\right] v(t)+\sum_{i_{1}=1}^{n_{1}} \mathscr{K}\left(k_{i_{1}}, L_{i_{1}} v\right)(t) \\
+\sum_{i_{2}=1}^{n_{2}} h_{i_{2}}(t) y_{i_{2}}+f(t), \quad t \in I_{T}, \\
M v(0)=M v_{0} .
\end{gathered}
$$

Here, $I_{T}=[0, T], \lambda_{0} \in \mathbf{C}, n_{1}, n_{2} \in \mathbf{N}, h_{i_{2}}: I_{T} \rightarrow \mathbf{C}, y_{i_{2}} \in X$, $i_{2}=1, \ldots, n_{2}$, whereas, $Z$ being another complex Banach space and $\mathscr{P}: Z \times X \rightarrow X$ being a bilinear bounded operator, $k_{i_{1}}: I_{T} \rightarrow Z$, and $\mathscr{K}\left(k_{i_{1}}, L_{i_{1}} v\right)(t)=\int_{0}^{t} \mathscr{P}\left(k_{i_{1}}(t-s), L_{i_{1}} v(s)\right) \mathrm{d} s$, $i_{1}=1, \ldots, n_{1}$. Of course, if $Z=\mathbf{C}$, then $\mathscr{P}$ may be the scalar multiplication in $X$. As $M, L$, and $L_{i_{1}}, i_{1}=1, \ldots, n_{1}$, we take closed single-valued linear operators from $X$ to itself, whose domains fulfill the relation $\mathscr{D}(L) \subseteq \bigcap_{i_{1}=1}^{n_{1}}\left[\mathscr{D}(M) \cap \mathscr{D}\left(L_{i_{1}}\right)\right]$, and we require $L$ to have a bounded inverse, allowing $M$ to be not invertible. Hence, in general, $A=L M^{-1}$ is only a multivalued linear operator in $X$ having domain $\mathscr{D}(A)=$ $M(\mathscr{D}(L))$. Assuming that $A$ satisfies (2) and that the data $k_{i_{1}}$, $h_{i_{2}}, y_{i_{2}}$ and $f, i_{l}=1, \ldots, n_{l}, l=1,2$, are suitably chosen, problem (3) is then reduced to an equivalent fixed pointequation for the new unknown $w=L\left(v-v_{0}\right), v_{0} \in \mathscr{D}(L)$. It is here that the results of Sections 3 and 4 play their role, leading us to Theorem 48. In that theorem, provided that $5 \alpha+2 \beta>6$, we will prove that if $k_{i_{1}} \in C^{\eta_{i_{1}}}\left(I_{T} ; Z\right), h_{i_{2}} \in C^{\sigma_{i_{2}}}$ $\left(I_{T} ; \mathbf{C}\right), y_{i_{2}} \in Y_{\gamma_{i_{2}}}^{r}, Y_{\gamma_{i_{2}}}^{r} \in\left\{(X, \mathscr{D}(A))_{\gamma_{i_{2}}, r}, X_{A}^{\gamma_{i_{2}}, r}\right\}$, and $f \in C^{\mu}$ $\left(I_{T} ; X\right)$ for opportunely chosen $\eta_{i_{1}}, \sigma_{i_{2}}, \gamma_{i_{2}}$, and $\mu, i_{l}=$ $1, \ldots, n_{l}, l=1,2$, then problem (3) has a unique strict solution $v \in C^{\tau}\left(I_{T} ; \mathscr{D}(L)\right)$ satisfying $v(0)=v_{0}$ and $L v, \mathrm{~d} M v / \mathrm{d} t \in C^{\tau}\left(I_{T} ; X\right)$, where $\tau=\min _{i_{l}=1, \ldots, n_{l}, l=1,2}\left\{\eta_{i_{1}}, \sigma_{i_{2}}\right\}$ (cf. Remark 51). Section 5 concludes with applications of Theorem 48 to integral and nonintegral subcases of (3), (cf. Theorems 52-54 and 56). We stress that Theorem 48 repairs, generalizes, and improves [20, Theorems 5.6 and 5.7], where similar results were proven only for the case $\left(n_{1}, n_{2}, Y_{\psi}^{p}\right)=$ $\left(1,1, X_{A}^{\psi, p}\right)$ and under the stronger condition $3 \alpha+8 \beta>10$.

In Section 6, we give an application of Theorem 48 to a concrete case of problem (3) arising in the theory of heat conduction for materials with memory. In particular, we show how Theorem 48 characterizes the appropriate functional framework where to search for the solution of the inverse problem of recovering both $v$ and the vector $\left(k_{1}, \ldots, k_{r_{1}}\right), r_{1} \leq$ $n_{1}$, in (3) with $\left(i_{2}, n_{2}\right)=\left(i_{1}, n_{1}\right)$ and $h_{i_{1}}=k_{i_{1}}, i_{1}=1, \ldots, n_{1}$.

Finally, in the Appendix we explain how to amend [20, Theorems 5.6 and 5.7] in accordance to Theorem 48.

\section{Multivalued Linear Operators, Singular Semigroups, and the Spaces $(X, \mathscr{D}(A))_{\gamma, p}$ and $X_{A}^{\gamma, p}$}

Let $X$ be a complex Banach space endowed with norm $\|\cdot\|_{X}$ and let $\mathscr{P}(X)$ be the collection of all the subsets of $X$. For a number $\lambda \in \mathbf{C}$ and elements $\mathscr{U}, \mathscr{V}, \mathscr{W} \in \mathscr{P}(X) \backslash \emptyset, \lambda \mathscr{U}$, and $\mathscr{V}+\mathscr{W}$ denote the subsets of $X$ defined by $\{\lambda u: u \in \mathscr{U}\}$ and $\{v+w: v \in \mathscr{V}, w \in \mathscr{W}\}$, respectively. Then, a mapping $A$ from $X$ into $\mathscr{P}(X)$ is called a multivalued linear operator in $X$ if its domain $\mathscr{D}(A)=\{x \in X: A x \neq \emptyset\}$ is a linear subspace of $X$ and $A$ satisfies the following: (i) $A x+A y \subset A(x+y)$, for all $x, y \in \mathscr{D}(A)$; (ii) $\lambda A x \subset A(\lambda x)$, for all $\lambda \in \mathbf{C}$, for all $x \in \mathscr{D}(A)$. From now on, the shortening $\mathrm{m}$. l. will be always used for multivalued linear.

The set $\mathscr{R}(A)=\bigcup_{x \in \mathscr{D}(A)} A x$ is called the range of $A$. If $\mathscr{R}(A)=X$, then $A$ is said to be surjective. The following properties of a m. l. operator $A$ are immediate consequences of its definition (cf. [1, Theorems 2.1 and 2.2]): (iii) $A x+A y=$ $A(x+y)$, for all $x, y \in \mathscr{D}(A)$; (iv) $\lambda A x=A(\lambda x)$, for all $\lambda \epsilon$ $\mathrm{C} \backslash\{0\}$, for all $x \in \mathscr{D}(A)$; (v) $A 0$ is a linear subspace of $X$ and $A x=y+A 0$ for any $y \in A x, x \in \mathscr{D}(A)$. In particular, $A$ is single-valued if and only if $A 0=\{0\}$.

If $A$ is an m. l. operator in $X$, then its inverse $A^{-1}$ is defined to be the operator having domain $\mathscr{D}\left(A^{-1}\right)=\mathscr{R}(A)$ such that $A^{-1} y=\{x \in \mathscr{D}(A): y \in A x\}, y \in \mathscr{D}\left(A^{-1}\right) . A^{-1}$ is an m. 1. operator in $X$ too, and $\left(A^{-1}\right)^{-1}=A$. The set $A^{-1} 0=\{x \in$ $\mathscr{D}(A): 0 \in A x\}$ is called the kernel of $A$ and denoted by $\mathcal{N}(A)$. If $\mathcal{N}(A)=\{0\}$; that is, if $A^{-1}$ is single-valued, then $A$ is said to be injective. Observe that (v) yields $A x=A 0$ if and only if $x \in \mathscr{N}(A)$.

Given $\mathcal{U} \in \mathscr{P}(X) \backslash \emptyset$, we write $A(\mathcal{U})=\bigcup_{u \in \mathscr{U} \cap \mathscr{D}(A)} A u$, so that, in particular, $A(X)=A(\mathscr{D}(A))=\mathscr{R}(A)$. If $A_{j}$, $j=1,2$ are m. 1 . operators in $X$ and $\lambda \in \mathbf{C}$, then the scalar multiplication $\lambda A_{1}$, the sum $A_{1}+A_{2}$, and the product $A_{1} A_{2}$ are defined by

$$
\begin{gathered}
\mathscr{D}\left(\lambda A_{1}\right)=\mathscr{D}\left(A_{1}\right), \\
\left(\lambda A_{1}\right) x=\lambda A_{1} x, \quad x \in \mathscr{D}\left(\lambda A_{1}\right), \\
\mathscr{D}\left(A_{1}+A_{2}\right)=\mathscr{D}\left(A_{1}\right) \cap \mathscr{D}\left(A_{2}\right), \\
\left(A_{1}+A_{2}\right) x=A_{1} x+A_{2} x, \quad x \in \mathscr{D}\left(A_{1}+A_{2}\right), \\
\mathscr{D}\left(A_{1} A_{2}\right)=\left\{x \in \mathscr{D}\left(A_{2}\right): A_{1}\left(A_{2} x\right) \neq \emptyset\right\}, \\
\left(A_{1} A_{2}\right) x=A_{1}\left(A_{2} x\right), \quad x \in \mathscr{D}\left(A_{1} A_{2}\right),
\end{gathered}
$$

where $\lambda A_{1}, A_{1}+A_{2}$ and $A_{1} A_{2}$ are m. l. operators in $X$ and $\left(A_{1} A_{2}\right)^{-1}=A_{2}^{-1} A_{1}^{-1}$.

Let $A$ and $B$ be m. l. operators in $X$. We write $A \subset B$ if $\mathscr{D}(A) \subseteq \mathscr{D}(B)$ and $A x \subseteq B x$ for every $x \in \mathscr{D}(A)$. Clearly, $A \subset$ $B \subset A$ if and only if $A=B$. If $A \subset B$ and $A x=B x$ for every $x \in \mathscr{D}(A)$, then $B$ is called an extension of $A$. If a linear singlevalued operator $S$ has domain $\mathscr{D}(S)=\mathscr{D}(A)$ and $S \subset A$, that is, $S x \in A x$ for every $x \in \mathscr{D}(A)$, then $S$ is called a section of $A$. With an arbitrary section $S$, it holds $A x=S x+A 0, x \in \mathscr{D}(A)$, and $\mathscr{R}(A)=\mathscr{R}(S)+A 0$, but this latter sum may or may not 
be direct (cf. [25, p. 14]). A method for constructing sections is provided in [25, Proposition I.5.2].

If $X_{j}, j=1,2$, are two complex Banach spaces, then the linear space of all bounded single-valued linear operators $L$ from $X_{1}=\mathscr{D}(L)$ to $X_{2}$ is denoted by $\mathscr{L}\left(X_{1} ; X_{2}\right)\left(\mathscr{L}\left(X_{1}\right)\right.$ if $\left.X_{1}=X_{2}\right)$ and it is equipped with the uniform operator norm $\|L\|_{\mathscr{L}\left(X_{1} ; X_{2}\right)}=\sup _{\|x\|_{X_{1}} \leq 1}\|L x\|_{X_{2}}=\inf _{K \geq 0}\left\{\|L x\|_{X_{2}} \leq K\|x\|_{X_{1}}\right.$ : $\left.x \in X_{1}\right\}$. Then the resolvent set $\rho(A)$ of a m. l. operator $A$ is defined to be the set $\left\{z \in \mathrm{C}:(z I-A)^{-1} \in \mathscr{L}(X)\right\}$, with $I$ being the identity operator in $X$. The basic properties of the resolvent set of single-valued linear operators hold the same for $\mathrm{m}$. l. operators. First, if $\rho(A) \neq \emptyset$, then $A$ is closed; that is, its graph $\{(x, y) \in X \times X: x \in \mathscr{D}(A), y \in A x\}$ is closed (cf. $[25$, p. 43]). Further (cf. [1, Theorem 2.6]), $\rho(A)$ is an open set and the operator function $z \in \rho(A) \rightarrow(z I-A)^{-1} \in \mathscr{L}(X)$ is holomorphic. Finally (cf. [1, formula (2.1)]), the resolvent equation $\left(\lambda_{2}-\lambda_{1}\right)\left(\lambda_{1} I-A\right)^{-1}\left(\lambda_{2} I-A\right)^{-1}=\left(\lambda_{1} I-A\right)^{-1}-\left(\lambda_{2} I-\right.$ $A)^{-1}, \lambda_{1}, \lambda_{2} \in \rho(A)$, is satisfied, too. Unlike the single-valued case, instead, for $z \in \rho(A)$ the following inclusions hold (cf. [1, Theorem 2.7]):

$$
(z I-A)^{-1} A \subset z(z I-A)^{-1}-I \subset A(z I-A)^{-1} .
$$

Then, in general, $z(z I-A)^{-1}-I, z \in \rho(A)$, is only a bounded section of the m. 1 . operator $A(z I-A)^{-1}$. Throughout this paper, we denote this bounded section by $A^{\circ}(z I-A)^{-1}$, but we warn the reader that here $A^{\circ}$ does not necessarily denote a section of $A$ itself. Of course, if $A$ is single-valued, then $A^{\circ}(z I-A)^{-1}$ reduces to $A(z I-A)^{-1}$. Notice that (5) implies that $(z I-A)^{-1} A, z \in \rho(A)$, is single-valued on $\mathscr{D}(A)$ and $(z I-$ $A)^{-1} A x=(z I-A)^{-1} y$ with any $y \in A x, x \in \mathscr{D}(A)$. Another difference with the single-valued case is that for every $z \in$ $\rho(A)$ it holds $\mathcal{N}\left((z I-A)^{-1}\right)=A 0$. Indeed, $\left((z I-A)^{-1}\right)^{-1} 0=$ $(z I-A) 0=A 0$. Therefore, in the m. l. case, $\{0\} \subsetneq \mathcal{N}((z I-$ $A)^{-1}$ ), $z \in \rho(A)$. However (cf. [24, Lemma 2.1]), if $0 \in \rho(A)$, then $\mathcal{N}\left(A^{\circ}(z I-A)^{-1}\right)=\{0\}$, and, in addition, $x \notin A 0$ if and only if $A^{\circ}(z I-A)^{-1} x \notin A 0, z \in \rho(A)$. We also recall that for every $\lambda_{1}, \lambda_{2} \in \rho(A)$ the following slight variants of the resolvent equation hold (cf. [24, Lemma 2.2]):

$$
\begin{gathered}
\left(\lambda_{2}-\lambda_{1}\right)\left(\lambda_{1} I-A\right)^{-1} A^{\circ}\left(\lambda_{2} I-A\right)^{-1} \\
=A^{\circ}\left(\lambda_{1} I-A\right)^{-1}-A^{\circ}\left(\lambda_{2} I-A\right)^{-1}, \\
\left(\lambda_{2}-\lambda_{1}\right) A^{\circ}\left(\lambda_{1} I-A\right)^{-1}\left(\lambda_{2} I-A\right)^{-1} \\
=A^{\circ}\left(\lambda_{1} I-A\right)^{-1}-A^{\circ}\left(\lambda_{2} I-A\right)^{-1} .
\end{gathered}
$$

In particular, if $0 \in \rho(A)$, then, since $A^{\circ}(0 I-A)^{-1}=-I$, the first in (6) with $\left(\lambda_{1}, \lambda_{2}\right)=(0, \lambda)$ yields $\lambda(-A)^{-1} A^{\circ}(\lambda I-A)^{-1}=$ $-I-A^{\circ}(\lambda I-A)^{-1}=-\lambda(\lambda I-A)^{-1}$; that is,

$$
A^{-1} A^{\circ}(\lambda I-A)^{-1}=(\lambda I-A)^{-1}, \quad \lambda \in \rho(A) .
$$

Let $(A, \mathscr{D}(A))$ be a m. l. operator in $X$ satisfying the following resolvent condition:

(H1) $\rho(A)$ contains a region $\Sigma_{\alpha}=\{z \in \mathbf{C}: \mathfrak{R e} z \geq$ $\left.-c(|\Im \mathrm{m} z|+1)^{\alpha}, \Im \mathrm{m} z \in \mathbf{R}\right\}$, $\alpha \in(0,1], c>0$, and for some exponent $\beta \in(0, \alpha]$ and constant $C>0$ the following estimate holds:

$$
\left\|(\lambda I-A)^{-1}\right\|_{\mathscr{L}(X)} \leq C(|\lambda|+1)^{-\beta}, \quad \forall \lambda \in \Sigma_{\alpha} .
$$

Introduce the family $\left\{\mathrm{e}^{t A}\right\}_{t \geq 0} \in \mathscr{L}(X)$ defined by $\mathrm{e}^{0 A}=I$ and

$$
\mathrm{e}^{t A}=\frac{1}{2 \pi i} \int_{\Gamma} \mathrm{e}^{t \lambda}(\lambda I-A)^{-1} \mathrm{~d} \lambda, \quad t>0,
$$

where $\Gamma \subsetneq \Sigma_{\alpha} \backslash\{z \in \mathbf{C}: \mathfrak{R e} z \geq 0\}$ is the contour parametrized by $\lambda=-c(|\eta|+1)^{\alpha}+i \eta, \eta \in(-\infty, \infty)$. Then (cf. [1, pp. $360,361]),\left\{\mathrm{e}^{t A}\right\}_{t \geq 0}$ is a semigroup on $X$, infinitely many times strongly differentiable for $t>0$ with

$$
\begin{aligned}
D_{t}^{k} \mathrm{e}^{t \mathrm{~A}}= & \frac{1}{2 \pi i} \int_{\Gamma} \lambda^{k} \mathrm{e}^{t \lambda}(\lambda I-A)^{-1} \mathrm{~d} \lambda, \\
& t>0, \quad k \in \mathbf{N}=\{1,2, \ldots\},
\end{aligned}
$$

where $D_{t}^{k}=\mathrm{d}^{k} / \mathrm{d} t^{k}$. In general, no analyticity should be expected for $\mathrm{e}^{t A}$. For if $\alpha<1$ in (H1), then $\Sigma_{\alpha}$ does not contain any sector $\Lambda_{\omega+\pi / 2}=\{z \in \mathbf{C} \backslash\{0\}:|\arg z|<\omega+$ $\pi / 2\}, \omega \in(0, \pi / 2)$, and [15, Theorem 5.3], which extends $e^{t A}$ analytically to the sector $\Lambda_{\omega}$ containing the positive real axis, is not applicable. We stress that (9) and $\mathcal{N}\left((z I-A)^{-1}\right)=A 0$, $z \in \rho(A)$, imply $A 0 \subseteq \mathcal{N}\left(\mathrm{e}^{t A}\right)$ for every $t>0$, whereas $\mathcal{N}\left(\mathrm{e}^{0 A}\right)=\mathcal{N}(I)=\{0\}$. Hence, if $A$ is really an $\mathrm{m}$. l. operator, then $\{0\} \subsetneq A 0 \subseteq \bigcap_{t>0} \mathcal{N}\left(\mathrm{e}^{t A}\right)$. From the semigroup property it also follows that $\mathscr{N}\left(\mathrm{e}^{t_{0} A}\right) \subseteq \mathcal{N}\left(\mathrm{e}^{t_{1} A}\right)$ for $t_{1} \geq t_{0} \geq 0$.

Now, for every $\theta \in \mathbf{C}$ such that $\mathfrak{R e} \theta \geq 0$ we set

$$
\left[(-A)^{\theta}\right]^{\circ} \mathrm{e}^{t A}=\frac{1}{2 \pi i} \int_{\Gamma}(-\lambda)^{\theta} \mathrm{e}^{t \lambda}(\lambda I-A)^{-1} \mathrm{~d} \lambda, \quad t>0 .
$$

Here, for the multivalued function $(-\lambda)^{\theta}=\mathrm{e}^{\theta \ln (-\lambda)}$ we choose the principal branch holomorphic in the region $\mathrm{C} \backslash\{z \in$ C : $\mathfrak{R e} z \geq 0\}$, where for principal branch we mean the principal determination $\ln |z|+i \arg (z)$ of $\operatorname{Ln}(z)$. We briefly recall the main properties of operators $\left[(-A)^{\theta}\right]^{\circ} \mathrm{e}^{t A}$. Of course, $\left[(-A)^{0}\right]^{\circ} \mathrm{e}^{t A}=\mathrm{e}^{t A}, t>0$. As shown in [26, p. 426], $\left[(-A)^{k}\right]^{\circ}$ $\mathrm{e}^{t A}, k \in \mathbf{N}, t>0$, is a section of $(-A)^{k} \mathrm{e}^{t A}$, so that from (10) we get

$$
(-1)^{k} D_{t}^{k} \mathrm{e}^{t A}=\left[(-A)^{k}\right]^{\circ} \mathrm{e}^{t A} \subset(-A)^{k} \mathrm{e}^{t A}, \quad t>0, k \in \mathbf{N} .
$$

Moreover (cf. [19, formula (22)] with $\theta \geq 0$ being replaced by $\mathfrak{R e} \theta \geq 0$ ), we get

$$
\begin{array}{r}
{\left[(-A)^{\theta}\right]^{\circ} \mathrm{e}^{t A}-\left[(-A)^{\theta}\right]^{\circ} \mathrm{e}^{s A}=-\int_{s}^{t}\left[(-A)^{\theta+1}\right]^{\circ} \mathrm{e}^{\xi A} \mathrm{~d} \xi,} \\
\operatorname{Re} \theta \geq 0, \quad 0<s<t .
\end{array}
$$

Finally, (H1) implies the following estimates (cf. [1, 24, Section 3]):

$$
\left\|\left[(-A)^{\theta}\right]^{\circ} \mathrm{e}^{t A}\right\|_{\mathscr{L}(X)} \leq \widetilde{c}_{\alpha, \beta, \theta} t^{(\beta-\Re \mathrm{R} \theta-1) / \alpha}, \quad \mathfrak{R e} \theta \geq 0, t>0,
$$


where the $\widetilde{c}_{\alpha, \beta, \theta}$ 's are positive constants depending on $\alpha, \beta$, and $\theta$. Thus, letting $\theta=0$ in (14), we see that if $\beta \in(0,1)$, then the operator function $t \in(0, \infty) \rightarrow \mathrm{e}^{t A} \in \mathscr{L}(X)$ may be singular at the origin and the semigroup is not necessarily strongly continuous in the $X$-norm on the closure $\overline{\mathscr{D}(A)}$ of $\mathscr{D}(A)$ in $X$. Notice that if $\alpha+\beta>1$, then the singularity is a weak one, in the sense that $\left\{\mathrm{e}^{t A}\right\}_{t \geq 0}$ is integrable in norm in any interval $[0, \tau], \tau>0$. Further (cf. [24, Lemma 3.9]), if $\alpha+\beta>1$, then $A 0=\bigcap_{t>0} \mathcal{N}\left(\mathrm{e}^{t A}\right)$, and if $\alpha=1$, then $A 0=$ $\mathcal{N}\left(\mathrm{e}^{t A}\right)$ for every $t>0$.

Observe that $A 0 \subseteq \mathcal{N}\left(\left[(-A)^{\theta}\right]^{\circ} \mathrm{e}^{t A}\right), \mathfrak{R e} \theta \geq 0, t>0$, so that $A 0 \subseteq \bigcap_{t>0} \mathcal{N}\left(\left[(-A)^{\theta}\right]^{\circ} \mathrm{e}^{t A}\right), \mathfrak{R e} \theta \geq 0$. The operators $\left[(-A)^{\theta}\right]^{\circ} \mathrm{e}^{t A}$ satisfy the following semigroup type property.

Proposition 1. Let $\theta_{j} \in \mathbf{C}, \mathfrak{R e} \theta_{j} \geq 0$, and let $t_{j}>0, j=1,2$. Then

$$
\left[(-A)^{\theta_{1}}\right]^{\circ} e^{t_{1} A}\left[(-A)^{\theta_{2}}\right]^{\circ} e^{t_{2} A}=\left[(-A)^{\theta_{1}+\theta_{2}}\right]^{\circ} e^{\left(t_{1}+t_{2}\right) A}
$$

Proof. First, the function $\lambda \in \rho(A) \rightarrow(-\lambda)^{\theta} e^{t \lambda}(\lambda I-A)^{-1} \epsilon$ $\mathscr{L}(X)$ being holomorphic for every $\mathfrak{R e} \theta \geq 0$ and $t>0$, and the contour $\Gamma$ in $(11)$ with $(\theta, t)=\left(\theta_{2}, t_{2}\right)$ can be replaced with the contour $\Gamma^{\prime} \mp \Sigma_{\alpha} \backslash\{z \in \mathbf{C}: \mathfrak{R e} z \geq 0\}$ parametrized by $\mu=-c^{\prime}(|\eta|+1)^{\alpha}+i \eta, \eta \in(-\infty, \infty), c^{\prime} \in(0, c)$, and lies to the right of $\Gamma$. Then, for every $x \in X$, from the resolvent equation we obtain

$$
\begin{aligned}
& {\left[(-A)^{\theta_{1}}\right]^{\circ} \mathrm{e}^{t_{1} A}\left[(-A)^{\theta_{2}}\right]^{\circ} \mathrm{e}^{t_{2} A} x} \\
& =\left(\frac{1}{2 \pi i}\right)^{2} \int_{\Gamma}(-\lambda)^{\theta_{1}} \mathrm{e}^{t_{1} \lambda} \\
& \times\left[\int_{\Gamma^{\prime}}(-\mu)^{\theta_{2}} \mathrm{e}^{t_{2} \mu}(\lambda I-A)^{-1}(\mu I-A)^{-1} x \mathrm{~d} \mu\right] \mathrm{d} \lambda \\
& =\left(\frac{1}{2 \pi i}\right)^{2} \int_{\Gamma}(-\lambda)^{\theta_{1}} \mathrm{e}^{t_{1} \lambda}(\lambda I-A)^{-1} \\
& \times\left[\left(\int_{\Gamma^{\prime}}(-\mu)^{\theta_{2}} \mathrm{e}^{t_{2} \mu}(\mu-\lambda)^{-1} \mathrm{~d} \mu\right) x\right] \mathrm{d} \lambda \\
& -\left(\frac{1}{2 \pi i}\right)^{2} \int_{\Gamma^{\prime}}(-\mu)^{\theta_{2}} \mathrm{e}^{t_{2} \mu}(\mu I-A)^{-1} \\
& \times\left[\left(\int_{\Gamma}(-\lambda)^{\theta_{1}} \mathrm{e}^{t_{1} \lambda}(\lambda-\mu)^{-1} \mathrm{~d} \lambda\right) x\right] \mathrm{d} \mu .
\end{aligned}
$$

Now, after having enclosed $\Gamma$ and $\Gamma^{\prime}$ on the left with an $\operatorname{arc} \Delta_{R}$ of the circle $\left\{z \in \mathbf{C}:\left|z+c^{\prime}\right|=R\right\}, R>c-c^{\prime}$, we apply the residue theorem and let $R$ go to infinity. To this purpose, we observe that since the contours $\Gamma$ and $\Gamma^{\prime}$ both lie in the halfplane $\left\{z \in \mathbf{C}: \mathfrak{R e} z \leq-c^{\prime}\right\}$, the arc $\Delta_{R}$ may be parametrized in polar coordinates by $\mathfrak{R e} z=-c^{\prime}+R \cos \varphi, \Im \mathrm{m} z=R \sin \varphi$, $\varphi \in(\pi / 2,3 \pi / 2)$. Then, for every $z \in \Delta_{R}$ we have

$$
\begin{aligned}
\left|(-z)^{\theta} \mathrm{e}^{t z}\right| & =|z|^{\Re \mathrm{R} \theta} \mathrm{e}^{-\Im \mathrm{m} \theta \arg (-z)} \mathrm{e}^{t \mathfrak{R} \mathrm{e} z} \\
& \leq\left(R+c^{\prime}\right)^{\Re \mathrm{e} \theta} \mathrm{e}^{(\pi / 2)|\Im \mathrm{m} \theta|} \mathrm{e}^{-t c^{\prime}} \mathrm{e}^{t R \cos \varphi} .
\end{aligned}
$$

Since $t>0$ and $\varphi \in(\pi / 2,3 \pi / 2)$, the right-hand side of the latter inequality goes to zero as $R$ goes to infinity, so that $\lim _{R \rightarrow \infty, z \in \Delta_{R}}(-z)^{\theta} \mathrm{e}^{t z}=0$ for every $\mathfrak{R e} \theta \geq 0$ and $t>0$. The residue theorem together with the fact that $\Gamma^{\prime}$ lies to the right of $\Gamma$ thus yields $\int_{\Gamma^{\prime}}(-\mu)^{\theta_{2}} \mathrm{e}^{t_{2} \mu}(\mu-\lambda)^{-1} \mathrm{~d} \mu=2 \pi i(-\lambda)^{\theta_{2}} \mathrm{e}^{t_{2} \lambda}$ and $\int_{\Gamma}(-\lambda)^{\theta_{1}} \mathrm{e}^{t_{1} \lambda}(\lambda-\mu)^{-1} \mathrm{~d} \lambda=0$. Replacing these identities in (16) and using the equality $(-\lambda)^{\theta_{1}}(-\lambda)^{\theta_{2}}=(-\lambda)^{\theta_{1}+\theta_{2}}$ which is satisfied for the principal branch of the function $(-\lambda)^{\theta}=$ $\mathrm{e}^{\theta \operatorname{Ln}(-\lambda)}$, we finally find

$$
\begin{aligned}
& {\left[(-A)^{\theta_{1}}\right]^{\circ} \mathrm{e}^{t_{1} A}\left[(-A)^{\theta_{2}}\right]^{\circ} \mathrm{e}^{t_{2} A} x} \\
& \quad=\frac{1}{2 \pi i} \int_{\Gamma}(-\lambda)^{\theta_{1}+\theta_{2}} \mathrm{e}^{\left(t_{1}+t_{2}\right) \lambda}(\lambda I-A)^{-1} x \mathrm{~d} \lambda .
\end{aligned}
$$

The right-hand side being precisely $\left[(-A)^{\theta_{1}+\theta_{2}}\right]^{\circ} \mathrm{e}^{\left(t_{1}+t_{2}\right) A} x$, the proof is complete.

For an m. l. operator $A$ satisfying $(\mathrm{H} 1)$ we introduce now the spaces $(X, \mathscr{D}(A))_{\gamma, p}$ and $X_{A}^{\gamma, p}$. We first specify a topology on $\mathscr{D}(A)$ equipping it with the norm $\|x\|_{\mathscr{D}(A)}=\inf _{y \in A x}\|y\|_{X}$, $x \in \mathscr{D}(A)$. Since $A^{-1} \in \mathscr{L}(X)$, this norm is equivalent to the graph norm and makes $\mathscr{D}(A)$ a complex Banach space (cf. [2, Proposition 1.11]). As $X_{1}$ and $X_{2}$ being given normed complex linear spaces, we will write $X_{1} \hookrightarrow X_{2}$ if $X_{1} \subseteq X_{2}$ and there exists a positive constant $C_{0}$ such that $\|x\|_{X_{2}} \leq C_{0}\|x\|_{X_{1}}$ for every $x \in X_{1}$. If $X_{1} \hookrightarrow X_{2} \hookrightarrow X_{1}$, that is, if $X_{1}=X_{2}$ and the norms $\|\cdot\|_{X_{1}}$ and $\|\cdot\|_{X_{2}}$ are equivalent, then we will write $X_{1} \cong X_{2}$. Of course, $\mathscr{D}(A)$ with the norm $\|\cdot\|_{\mathscr{D}(A)}$ satisfies $\mathscr{D}(A) \hookrightarrow X$. In fact, if $x \in \mathscr{D}(A)$, then for every $y \in A x$ we have $x=A^{-1} y$, so that $\|x\|_{X} \leq\left\|A^{-1}\right\|_{\mathscr{L}(X)}\|y\|_{X} \leq$ $C\|y\|_{X}$. Taking the infimum with respect to $y \in A x$, we thus find $\|x\|_{X} \leq C\|x\|_{\mathscr{D}(A)}$ for every $x \in \mathscr{D}(A)$. If $Y$ is a Banach space, we denote by $C((0, \infty) ; Y)$ the set of all continuos functions from $(0, \infty)$ to $Y$, and for a $Y$-valued strongly measurable function $g(\xi), \xi \in(0, \infty)$, we set $\|g(\xi)\|_{L_{q}^{*}(Y)}=$ $\left(\int_{0}^{\infty}\|g(\xi)\|_{Y}^{q}(\mathrm{~d} \xi / \xi)\right)^{1 / q}, q \in[1, \infty)$, and $\|g(\xi)\|_{L_{\infty}^{*}(Y)}=$ $\sup _{\xi \in(0, \infty)}\|g(\xi)\|_{Y}$. Let $p_{0}, p_{1} \in[1, \infty)$ or let $p_{0}=p_{1}=\infty$, and for $\gamma \in(0,1)$ define $p^{-1}=(1-\gamma) p_{0}^{-1}+\gamma p_{1}^{-1}$ if $p_{0}, p_{1} \in$ $[1, \infty)$ and $p=\infty$ if $p_{0}=p_{1}=\infty$. Let us set

$$
\begin{gathered}
(X, \mathscr{D}(A))_{\gamma, p} \\
=\left\{x \in X: x=v_{0}(\xi)+v_{1}(\xi), \xi \in(0, \infty),\right. \\
v_{0} \in C((0, \infty) ; X), v_{1} \in C((0, \infty) ; \mathscr{D}(A)), \\
\left.\left\|\xi^{\gamma} v_{0}(\xi)\right\|_{L_{p_{0}}^{*}(X)}+\left\|\xi^{\gamma-1} v_{1}(\xi)\right\|_{L_{p_{1}}^{*}(\mathscr{D}(A))}<\infty\right\}, \\
\|x\|_{(X, \mathscr{D}(A))_{\gamma, p}} \\
=\inf _{v_{0}, v_{1}}\left\{\left\|\xi^{\gamma} v_{0}(\xi)\right\|_{L_{p_{0}}^{*}(X)}+\left\|\xi^{\gamma-1} v_{1}(\xi)\right\|_{L_{p_{1}}^{*}(\mathscr{D}(A))}\right\} .
\end{gathered}
$$

This characterization of the spaces $(X, \mathscr{D}(A))_{\gamma, p}$ is that obtained by the so-called "mean-methods", and it is equivalent to that performed by the "K-method" (cf. [27, Theorem 1.5.2 and Remark 1.5.2/2]) and the "trace-method" 
(cf. [27, Theorem 1.8.2]). Then, due to [27, Theorem 1.3.3], for every $\gamma \in(0,1)$ and $p \in[1, \infty]$ the space $(X, \mathscr{D}(A))_{\gamma, p}$ is an exact real interpolation space of exponent $\gamma$ between $X$ and $\mathscr{D}(A)$. Observe that by exchanging the role of $X$ and $\mathscr{D}(A)$ and performing the transformation $\xi=\tau^{-1}$, we get $(X, \mathscr{D}(A))_{\gamma, p}=(\mathscr{D}(A), X)_{1-\gamma, p}$. Also, if $\mathscr{D}(A)=X$, then $(X, \mathscr{D}(A))_{\gamma, p} \cong X$ (cf. [27, Theorem 1.3.3(f)]). The definition of the spaces $(X, \mathscr{D}(A))_{\gamma, p}$ is meaningful even for the limiting cases $(\gamma, p)=(i, \infty), i=0,1$, whereas $(X, \mathscr{D}(A))_{i, p}, i=0,1$, $p \in[1, \infty)$, reduces to the zero element of $X$. In particular (cf. [28, pp. 10-15]), denoting by $\widetilde{Y}^{X}$ the completion of $\mathscr{D}(A)$ relative to $X$ and endowing it with the norm $\|\cdot\|_{\tilde{Y}^{X}}$ in [28, p. 14], we get $(X, \mathscr{D}(A))_{0, \infty} \cong X$ and $(X, \mathscr{D}(A))_{1, \infty} \cong \tilde{Y}^{X}$. Let $\gamma_{1} \in(0,1)$ and let $p_{j} \in[1, \infty], j=1,2$. Then, for $\gamma_{2} \in\left(0, \gamma_{1}\right)$ and $q_{j} \in\left[1, p_{j}\right], j=1,2$, the following chain of embeddings holds:

$$
\begin{aligned}
\mathscr{D}(A) & \hookrightarrow(X, \mathscr{D}(A))_{1, \infty} \hookrightarrow(X, \mathscr{D}(A))_{\gamma_{1}, 1} \\
& \hookrightarrow(X, \mathscr{D}(A))_{\gamma_{1}, q_{1}} \hookrightarrow(X, \mathscr{D}(A))_{\gamma_{1}, p_{1}} \\
& \hookrightarrow(X, \mathscr{D}(A))_{\gamma_{2}, 1} \hookrightarrow(X, \mathscr{D}(A))_{\gamma_{2}, q_{2}} \\
& \hookrightarrow(X, \mathscr{D}(A))_{\gamma_{2}, p_{2}} \hookrightarrow \overline{\mathscr{D}(A)}
\end{aligned}
$$

Let $\gamma \in[0,1]$. Recall that a Banach space $E$ is said to be of class $J(\gamma, X, \mathscr{D}(A)) \cap K(\gamma, X, \mathscr{D}(A))$ and shortened to $E \in J(\gamma) \cap$ $K(\gamma)$, if $E$ is an intermediate space between $(X, \mathscr{D}(A))_{\gamma, \infty}$ and $(X, \mathscr{D}(A))_{\gamma, 1}$, that is, if $(X, \mathscr{D}(A))_{\gamma, 1} \hookrightarrow E \hookrightarrow(X, \mathscr{D}(A))_{\gamma, \infty}$. From (20) it thus follows that $(X, \mathscr{D}(A))_{\gamma, p} \in J(\gamma) \cap K(\gamma)$, for every $\gamma \in(0,1)$ and $p \in[1, \infty]$. Moreover, since $(X, \mathscr{D}(A))_{i, 1}=\{0\}, i=0,1$, and $(X, \mathscr{D}(A))_{0, \infty} \cong X$, we have $\mathscr{D}(A) \in J(1) \cap K(1)$ and $X \in J(0) \cap K(0)$. Then (cf. [28, p. 12], [27, Theorem 1.10.2], and [9, Section 1.2.3]), for $\gamma_{j} \in(0,1)$ and $p_{j} \in[1, \infty], j=0,1,2$, the reiteration theorem yields

$$
\begin{gathered}
\left((X, \mathscr{D}(A))_{\gamma_{1}, p_{1}},(X, \mathscr{D}(A))_{\gamma_{2}, p_{2}}\right)_{\gamma_{0}, p_{0}} \\
\cong(X, \mathscr{D}(A))_{\left(1-\gamma_{0}\right) \gamma_{1}+\gamma_{0} \gamma_{2}, p_{0}}, \\
\left((X, \mathscr{D}(A))_{\gamma_{1}, p_{1}}, \mathscr{D}(A)\right)_{\gamma_{0}, p_{0}} \cong(X, \mathscr{D}(A))_{\left(1-\gamma_{0}\right) \gamma_{1}+\gamma_{0}, p_{0}}, \\
\left(X,(X, \mathscr{D}(A))_{\gamma_{2}, p_{2}}\right)_{\gamma_{0}, p_{0}} \cong(X, \mathscr{D}(A))_{\gamma_{0} \gamma_{2}, p_{0}} .
\end{gathered}
$$

Finally (cf. [29, Theorem 1.II and Remark 1.III]), we recall that if $X_{1}$ and $X_{2}$ are two complex Banach spaces and $T \epsilon$ $\mathscr{L}\left(X_{1} ; X_{2}\right)$ is such that $T \in \mathscr{L}\left(Y_{1_{k}} ; Y_{2_{k}}\right), Y_{j_{k}} \subseteq X_{j}, j, k=1,2$, then $T \in \mathscr{L}\left(\left(Y_{1_{1}}, Y_{1_{2}}\right)_{\gamma_{0}, p_{0}} ;\left(Y_{2_{1}}, Y_{2_{2}}\right)_{\gamma_{0}, p_{0}}\right), \gamma_{0} \in(0,1), p_{0} \in$ $[1, \infty]$, and

$$
\|T\|_{\mathscr{L}\left(\left(Y_{1_{1}}, Y_{1_{2}}\right)_{\gamma_{0}, p_{0}} ;\left(Y_{2_{1}}, Y_{2_{2}}\right)_{\gamma_{0}, p_{0}}\right)} \leq\|T\|_{\mathscr{L}\left(Y_{1_{1}} ; Y_{2_{1}}\right)}^{1-\gamma_{0}}\|T\|_{\mathscr{L}\left(Y_{1_{2}} ; Y_{2_{2}}\right)}^{\gamma_{0}} .
$$

As a consequence of this general result and the identity

$$
\left((X, \mathscr{D}(A))_{\gamma_{1}, p_{1}}, X\right)_{\gamma_{0}, p_{0}}=\left(X,(X, \mathscr{D}(A))_{\gamma_{1}, p_{1}}\right)_{1-\gamma_{0}, p_{0}},
$$

from the third in (21) we find that if $T \in \mathscr{L}(X)$ is such that $T \in \mathscr{L}\left(X ;(X, \mathscr{D}(A))_{\gamma_{1}, p_{1}}\right)$ and $T \in \mathscr{L}\left((X, \mathscr{D}(A))_{\gamma_{2}, p_{2}} ; X\right)$, then $T \in \mathscr{L}\left((X, \mathscr{D}(A))_{\gamma_{0} \gamma_{2}, p_{0}} ;(X, \mathscr{D}(A))_{\left(1-\gamma_{0}\right) \gamma_{1}, p_{0}}\right), \gamma_{j} \in(0,1)$, $p_{j} \in[1, \infty], j=0,1,2$, and the following estimate holds:

$$
\begin{aligned}
& \|T\|_{\mathscr{L}\left((X, \mathscr{D}(A))_{\gamma_{0} \gamma_{2}, p_{0}} ;(X, \mathscr{D}(A))_{\left(1-\gamma_{0}\right) \gamma_{1}, p_{0}}\right)} \\
& \quad \leq\|T\|_{\mathscr{L}\left(X ;(X, \mathscr{D}(A))_{\gamma_{1}, p_{1}}\right)}^{1-\gamma_{0}}\|T\|_{\mathscr{L}\left((X, \mathscr{D}(A))_{\gamma_{2}, p_{2}} ; X\right)}^{\gamma_{0}} .
\end{aligned}
$$

Notice that here $\gamma_{0} \gamma_{2}+\left(1-\gamma_{0}\right) \gamma_{1} \in\left(\min \left\{\gamma_{1}, \gamma_{2}\right\}, \max \left\{\gamma_{1}, \gamma_{2}\right\}\right) \mp$ $(0,1)$ for every $\gamma_{0} \in(0,1)$. Therefore, if we let $\gamma=\gamma_{0} \gamma_{2}$ and let $\delta=\left(1-\gamma_{0}\right) \gamma_{1}$, then $\gamma+\delta<1, \gamma_{1}=\delta /\left(1-\gamma_{0}\right)>\delta$, and $\gamma_{2}=$ $\gamma / \gamma_{0}>\gamma$. Hence, in order that the additional inequalities $\gamma_{j}<$ $1, j=1,2$, are satisfied, we have to choose $\gamma_{0} \in(\gamma, 1-\delta)$. As we will see this simple observation will be the key for the proof of the second estimates (90) in the following Proposition 16.

We recall that for every fixed $x \in \mathscr{D}(A)$ the map $T(\lambda)=$ $\lambda x$ satisfies $\|T\|_{\mathscr{L}(\mathbf{C} ; X)}=\|x\|_{X},\|T\|_{\mathscr{L}(\mathbf{C} ; \mathscr{D}(A))}=\|x\|_{\mathscr{D}(A)}$ and $\|T\|_{\mathscr{L}\left(\mathbf{C},(X, \mathscr{D}(A))_{\gamma, p}\right)}=\|x\|_{(X, \mathscr{D}(A))_{\gamma, p}}$. Then $(22)$ with $X_{1}=Y_{1_{1}}=$ $Y_{1_{2}}=\mathrm{C}, X_{2}=Y_{2_{1}}=X$ and $Y_{2_{2}}=\mathscr{D}(A)$ yields the interpolation inequality:

$$
\begin{array}{r}
\|x\|_{(X, \mathscr{D}(A))_{\gamma, p}} \leq \mathcal{c}_{0}\|x\|_{X}^{1-\gamma}\|x\|_{\mathscr{D}(A)}^{\gamma}, \\
x \in \mathscr{D}(A), \quad \gamma \in(0,1), \quad p \in[1, \infty],
\end{array}
$$

with $c_{0}$ being the positive constant depending on $\gamma$ and $p$ such that $\|\lambda\|_{(\mathbf{C}, \mathbf{C})_{\gamma, p}} \leq c_{0}|\lambda|$.

As another application of (22) and for further needs, we also recall that if $A$ satisfies ( $\mathrm{H} 1)$, then $A^{\circ}(z I-A)^{-1}$ satisfies the estimate (cf. [24, formulae (4.16) and (4.17)]).

Consider

$$
\begin{gathered}
\left\|A^{\circ}(z I-A)^{-1}\right\|_{\mathscr{L}(X)} \leq(C+1)(|z|+1)^{1-\beta}, \quad \forall z \in \Sigma_{\alpha}, \\
\left\|A^{\circ}(z I-A)^{-1}\right\|_{\mathscr{L}(\mathscr{D}(A) ; X)} \leq C(|z|+1)^{-\beta}, \quad \forall z \in \Sigma_{\alpha} .
\end{gathered}
$$

From (26), using (22) with $X_{j}=Y_{j_{1}}=Y_{2_{2}}=X, j=1,2$, and $Y_{1_{2}}=\mathscr{D}(A)$, it then follows for every $\gamma \in(0,1)$ and $p \in[1, \infty]$

$$
\begin{aligned}
& \left\|A^{\circ}(z I-A)^{-1}\right\|_{\mathscr{L}\left((X, \mathscr{D}(A))_{\gamma, p} ; X\right)} \\
& \quad \leq c_{1}(C+1)^{1-\gamma} C^{\gamma}(|z|+1)^{1-\beta-\gamma}, \quad \forall z \in \Sigma_{\alpha},
\end{aligned}
$$

where $c_{1}$ is the positive constant depending on $\gamma$ and $p$ such that $\|x\|_{X} \leq c_{1}\|x\|_{(X, X)_{\gamma, p}}$.

For $\gamma \in(0,1)$ and $p \in[1, \infty]$ we now define the Banach spaces $X_{A}^{\gamma, p}$ by

$$
\begin{gathered}
X_{A}^{\gamma, p}=\left\{x \in X:[x]_{X_{A}^{\gamma, p}}:=\left\|\xi^{\gamma} A^{\circ}(\xi I-A)^{-1} x\right\|_{L_{p}^{*}(X)}<\infty\right\}, \\
\|x\|_{X_{A}^{\gamma, p}}=\|x\|_{X}+[x]_{X_{A}^{\gamma, p} .}
\end{gathered}
$$

It is a well-known fact that if $A$ is single-valued and $\beta=1$ in (H1), then $(X, \mathscr{D}(A))_{\gamma, p} \cong X_{A}^{\gamma, p}$ (cf. [30, Theorem 3.1] and [27, Theorem 1.14.2]). On the contrary, if $\beta \in(0,1)$, then such 
an equivalence is no longer true, as first observed in [13, Theorem 2] for single-valued operators and, in the case $p=\infty$, in [2, Theorem 1.12] for the m. l. ones. Recently, extending [13] to $\mathrm{m}$. 1 . operators and [2] to $p \in[1, \infty]$, in [24, Proposition 4.3] it has been shown that the following embedding relations hold:

$$
\begin{gathered}
X_{A}^{\gamma, p} \hookrightarrow(X, \mathscr{D}(A))_{\gamma, p}, \quad \gamma \in(0,1), p \in[1, \infty], \\
(X, \mathscr{D}(A))_{\gamma, p} \hookrightarrow X_{A}^{\gamma+\beta-1, p}, \quad \gamma \in(1-\beta, 1), p \in[1, \infty] .
\end{gathered}
$$

Then, as in the single-valued case, $(X, \mathscr{D}(A))_{\gamma, p} \cong X_{A}^{\gamma, p}$ if $\beta=$ 1 in (H1). More precisely (see the proof of [24, Proposition 4.3]), if $x \in X_{A}^{\gamma, p}, \gamma \in(0,1), p \in[1, \infty]$, then

$$
\|x\|_{(X, \mathscr{D}(A))_{\gamma, p}} \leq 2\|x\|_{X_{A}^{\gamma, p}}
$$

whereas if $x \in(X, \mathscr{D}(A))_{\gamma, p}, \gamma \in(1-\beta, 1), p \in[1, \infty]$, then

$$
\|x\|_{X_{A}^{\gamma+\beta-1, p}} \leq c_{2}\|x\|_{(X, \mathscr{D}(A))_{\gamma, p}},
$$

with $c_{2}$ being a positive constant depending on $\beta, \gamma$ and $p$.

By setting $\delta=\gamma+\beta-1, \gamma \in(1-\beta, 1)$, from (30) it follows

$$
\begin{array}{r}
\mathscr{D}(A) \hookrightarrow(X, \mathscr{D}(A))_{1+\delta-\beta, p} \hookrightarrow X_{A}^{\delta, p} \hookrightarrow X, \\
\delta \in(0, \beta), \quad p \in[1, \infty] .
\end{array}
$$

Then, if $\beta \in(0,1)$, the spaces $X_{A}^{\delta, p}, \delta \in(0,1), p \in[1, \infty]$, are intermediate spaces between $X$ and $\mathscr{D}(A)$ only for $\delta \in(0, \beta)$, whereas, when $\delta \in[\beta, 1)$, they may be smaller than $\mathscr{D}(A)$. In any case, when $\beta \in(0,1)$, it is not known if the spaces $X_{A}^{\delta, p}, \delta \in(0, \beta), p \in[1, \infty]$, are only intermediate or just interpolation spaces between $X$ and $\mathscr{D}(A)$.

Notice that $\left[X_{A}^{\gamma, p} \cap A 0\right]=\{0\}, \gamma \in(0,1), p \in[1, \infty]$. Indeed, assume that there exists $x \neq 0$ such that $x \in\left[X_{A}^{\gamma, p} \cap\right.$ $A 0]$ for some $\gamma \in(0,1)$ and $p \in[1, \infty]$. Then, since $x \in A 0=$ $\mathcal{N}\left((z I-A)^{-1}\right), z \in \rho(A)$, we have $A^{\circ}(\xi I-A)^{-1} x=\xi(\xi-$ $A)^{-1} x-x=-x$ for every $\xi>0$ and $[x]_{X_{A}^{\gamma, p}}=\left\|\xi^{\gamma}\right\|_{L_{p}^{*}(X)}\|x\|_{X}=$ $\infty$, contradicting $x \in X_{A}^{\gamma, p}$. This property plays a key role in the proof of many of the results in [24]. Further, due to (30), it implies that $[\mathscr{D}(A) \cap A 0]=\left[(X, \mathscr{D}(A))_{\gamma, p} \cap A 0\right]=\{0\}, \gamma \in$ $(1-\beta, 1), p \in[1, \infty]$. On the contrary, since $\{0\}$ may be a proper subset of $\left[(X, \mathscr{D}(A))_{\gamma, p} \cap A 0\right]$ for $\gamma \in(0,1-\beta], \beta<1$, in general it is not true that $[\overline{\mathscr{D}(A)} \cap A 0]=\{0\}$. This is true, instead, if $\beta=1$. In this case the topological direct sum $X_{0}=$ $\overline{\mathscr{D}(A)} \oplus A 0$ is a closed subspace of $X$, and if $X$ is reflexive, it coincides with the whole $X$ (cf. [3, Theorems 2.4 and 2.6]).

For every $\gamma \in(0,1)$ and $p \in[1, \infty]$ from (27), (29), and (31) it follows

$$
\begin{aligned}
& \left\|A^{\circ}(z I-A)^{-1}\right\|_{\mathscr{L}\left(X_{A}^{\gamma, p} ; X\right)} \\
& \quad \leq 2 c_{1}(C+1)^{1-\gamma} C^{\gamma}(|z|+1)^{1-\beta-\gamma}, \quad \forall z \in \Sigma_{\alpha} .
\end{aligned}
$$

Hence, for $\gamma \in(0,1)$ and $p \in[1, \infty]$ we may rewrite $(27)$ and (34) more compactly as

$$
\left\|A^{\circ}(z I-A)^{-1}\right\|_{\mathscr{L}\left(Y_{\gamma}^{p} ; X\right)} \leq c_{3}(|z|+1)^{1-\beta-\gamma}, \quad \forall z \in \Sigma_{\alpha},
$$

where $Y_{\gamma}^{p} \in\left\{(X, \mathscr{D}(A))_{\gamma, p}, X_{A}^{\gamma, p}\right\}$ and $c_{3}$ is equal to $c_{1}(C+$ $1)^{1-\gamma} C^{\gamma}$ or $2 c_{1}(C+1)^{1-\gamma} C^{\gamma}$ according that $Y_{\gamma}^{p}=(X, \mathscr{D}(A))_{\gamma, p}$ or $Y_{\gamma}^{p}=X_{A}^{\gamma, p}$.

With the exception of the case $\beta=1$, in general it is not clear if embeddings analogous to (20) hold even for the spaces $X_{A}^{\gamma, p}$. In fact, using (20), (29), and (30) we can only prove that if $\gamma \in(1-\beta, 1)$ and $1 \leq q \leq p \leq \infty$, then

$$
X_{A}^{\gamma, q} \hookrightarrow(X, \mathscr{D}(A))_{\gamma, q} \hookrightarrow(X, \mathscr{D}(A))_{\gamma, q} \hookrightarrow X_{A}^{\gamma+\beta-1, p},
$$

whereas if $1-\beta<\gamma_{2}<\gamma_{1}<1$ and $p_{1}, p_{2} \in[1, \infty]$, then

$$
X_{A}^{\gamma_{1}, p_{1}} \hookrightarrow(X, \mathscr{D}(A))_{\gamma_{1}, p_{1}} \hookrightarrow(X, \mathscr{D}(A))_{\gamma_{2}, p_{2}} \hookrightarrow X_{A}^{\gamma_{2}+\beta-1, p_{2}} .
$$

What can be proved without invoking (20), (29), and (30) and using only the definition of the norm $\|\cdot\|_{X_{A}^{\gamma, p}}$ is instead the following result, which extends to the spaces $X_{A}^{\gamma, p}$ the embeddings $(X, \mathscr{D}(A))_{\gamma_{1}, p} \hookrightarrow(X, \mathscr{D}(A))_{\gamma_{2}, p}$, and $(X, \mathscr{D}(A))_{\gamma_{1}, \infty} \hookrightarrow$ $(X, \mathscr{D}(A))_{\gamma_{2}, p}, 0<\gamma_{2}<\gamma_{1}<1, p \in[1, \infty]$ (cf. (20) with $\left(p_{1}, p_{2}\right)=(p, p)$ and $\left.\left(p_{1}, p_{2}\right)=(\infty, p)\right)$.

Proposition 2. Let A be an $\mathrm{m}$. l. operator satisfying the resolvent condition (H1). Then the following embeddings hold for every $0<\gamma_{2}<\gamma_{1}<1$ and $p \in[1, \infty]$ :

$$
\begin{gathered}
X_{A}^{\gamma_{1}, p} \hookrightarrow X_{A}^{\gamma_{2}, p}, \\
X_{A}^{\gamma_{1}, \infty} \hookrightarrow X_{A}^{\gamma_{2}, p} .
\end{gathered}
$$

Proof. If $\beta=1$ in (H1), then there is nothing to prove since $(X, \mathscr{D}(A))_{\gamma, p} \cong X_{A}^{\gamma, p}$ and both (38) and (39) follow from (20). Therefore, without loss of generality, we assume that $\beta \in$ $(0, \alpha]$ is such that $\beta<\alpha$ if $\alpha=1$. We begin by proving (38). Let first $p \in[1, \infty)$. For every $x \in X_{A}^{\gamma_{1}, p}, 0<\gamma_{2}<\gamma_{1}<1$, we write

$$
[x]_{X_{A}^{\gamma_{2}, p}}^{p}=I_{1}+I_{2}
$$

where

$$
I_{j}=\int_{a_{j}}^{b_{j}}\left\|\xi^{\gamma_{2}} A^{\circ}(\xi I-A)^{-1} x\right\|_{X}^{p} \frac{\mathrm{d} \xi}{\xi}, \quad j=1,2,
$$

$\left(a_{1}, b_{1}, a_{2}, b_{2}\right)=(0,1,1, \infty)$. Using the first inequality in (26) we find

$$
\begin{aligned}
I_{1} & \leq(C+1)^{p}\|x\|_{X}^{p} \int_{0}^{1} \xi^{\gamma_{2} p-1}(\xi+1)^{(1-\beta) p} \mathrm{~d} \xi \\
& \leq 2^{(1-\beta) p}(C+1)^{p}\|x\|_{X}^{p} \int_{0}^{1} \xi^{\gamma_{2} p-1} \mathrm{~d} \xi \leq\left[c_{4}\|x\|_{X_{A}^{\gamma_{1}, p}}\right]^{p},
\end{aligned}
$$

where $c_{4}=2^{1-\beta}(C+1)\left(\gamma_{2} p\right)^{-1 / p}$. Concerning $I_{2}$, instead, using $\gamma_{2}-\gamma_{1}<0$, we get

$$
\begin{aligned}
I_{2} & =\int_{1}^{\infty} \xi^{\left(\gamma_{2}-\gamma_{1}\right) p}\left\|\xi^{\gamma_{1}} A^{\circ}(\xi I-A)^{-1} x\right\|_{X}^{p} \frac{\mathrm{d} \xi}{\xi} \\
& \leq \int_{1}^{\infty}\left\|\xi^{\gamma_{1}} A^{\circ}(\xi I-A)^{-1} x\right\|_{X}^{p} \frac{\mathrm{d} \xi}{\xi} \leq[x]_{X_{A}^{\gamma_{1}, p}}^{p} \leq\|x\|_{X_{A}^{\gamma_{1}, p}}^{p} .
\end{aligned}
$$


Summing up (40)-(43) and setting $c_{5}=\left[\left(c_{4}\right)^{p}+1\right]^{1 / p}$, it thus follows $\|x\|_{X_{A}^{\gamma_{2}, p}}=\|x\|_{X}+[x]_{X_{A}^{\gamma_{2}, p}} \leq\left(1+c_{5}\right)\|x\|_{X_{A}^{\gamma_{1}, p}}$, completing the proof of $(38)$ in the case $p \in[1, \infty)$. Let $p=\infty$. For every $x \in X_{A}^{\gamma_{1}, \infty}, 0<\gamma_{2}<\gamma_{1}<1$, we write

$$
[x]_{X_{A}^{\gamma_{2}, \infty}}=\max \left\{I_{3}, I_{4}\right\},
$$

where $I_{j}=\sup _{\xi \in U_{j}}\left\|\xi^{\gamma_{2}} A^{\circ}(\xi I-A)^{-1} x\right\|_{X}, j=3,4, U_{3}=(0,1)$, $U_{4}=[1, \infty)$. Again, the first inequality in (26) yields

$$
\begin{aligned}
I_{3} & \leq(C+1)\|x\|_{X} \sup _{\xi \in(0,1)}\left[\xi^{\gamma_{2}}(\xi+1)^{1-\beta}\right] \\
& \leq 2^{1-\beta}(C+1)\|x\|_{X_{A}^{\gamma_{1}, \infty}} .
\end{aligned}
$$

Instead, using $\gamma_{2}-\gamma_{1}<0$, we have

$$
\begin{aligned}
I_{4} & =\sup _{\xi \in[1, \infty)} \xi^{\gamma_{2}-\gamma_{1}}\left\|\xi^{\gamma_{1}} A^{\circ}(\xi I-A)^{-1} x\right\|_{X} \leq[x]_{X_{A}^{\gamma_{1}, \infty}} \\
& \leq\|x\|_{X_{A}^{\gamma_{1}, \infty}} .
\end{aligned}
$$

Summing up (44)-(46) and setting $c_{6}=2^{1-\beta}(C+1)$, we thus find $\|x\|_{X_{A}^{\gamma_{2}, \infty}}=\|x\|_{X}+[x]_{X_{A}^{\gamma_{2}, \infty}} \leq\left(1+c_{6}\right)\|x\|_{X_{A}^{\gamma_{1}, \infty}}$. This completes the proof of (38) for the case $p=\infty$. We now prove (39). Due to (38) with $p=\infty$, it suffices to assume that $p \in$ $[1, \infty)$. As above, for every $x \in X_{A}^{\gamma_{1}, \infty}, 0<\gamma_{2}<\gamma_{1}<1$, we write $[x]_{X_{A}^{\gamma_{2}, p}}^{p}=I_{1}+I_{2}$, where $I_{1}$ and $I_{2}$ are defined by (41). Hence, the same computations as in (42) yield

$$
I_{1} \leq\left[c_{4}\|x\|_{X_{A}^{\gamma_{1}, \infty}}\right]^{p} \text {. }
$$

As far as $I_{2}$ is concerned, instead, we have

$$
I_{2} \leq[x]_{X_{A}^{\gamma_{1}, \infty}}^{p} \int_{1}^{\infty} \xi^{\left(\gamma_{2}-\gamma_{1}\right) p-1} \mathrm{~d} \xi \leq\left[\mathcal{c}_{7}\|x\|_{X_{A}^{\gamma_{1}, \infty}}\right]^{p}
$$

where $c_{7}=\left[\left(\gamma_{1}-\gamma_{2}\right) p\right]^{-1 / p}$. Summing up (47) and (48) and setting $c_{8}=\left[\left(c_{4}\right)^{p}+\left(c_{7}\right)^{p}\right]^{1 / p}$, we deduce $\|x\|_{X_{A}^{\gamma_{2}, p}} \leq(1+$ $\left.c_{8}\right)\|x\|_{X_{A}^{\gamma_{1}, \infty}}$. The proof is complete.

Remark 3. Notice that (37) with $p_{1}=p_{2}=p$ yields $X_{A}^{\gamma_{1}, p} \hookrightarrow$ $X_{A}^{\gamma_{2}+\beta-1, p}, 1-\beta<\gamma_{2}<\gamma_{1}<1$, and this latter embedding is less accurate than (38).

Remark 4. The main problem for extending (20) to the spaces $X_{A}^{\gamma, p}$ in the case $\beta<1$ is that it is not clear if it holds $X_{A}^{\gamma, q} \hookrightarrow$ $X_{A}^{\gamma, p}, 1 \leq q<p \leq \infty$. In fact, the embedding

$$
\begin{array}{r}
(X, \mathscr{D}(A))_{\gamma, q} \hookrightarrow(X, \mathscr{D}(A))_{\gamma, q}, \\
\gamma \in(0,1), \quad 1 \leq q<p \leq \infty,
\end{array}
$$

is a consequence of the property of the functional $K$ entering the definition of the interpolation spaces $(X, \mathscr{D}(A))_{\gamma, p}$ through the "K-method", and in particular of its monotonicity (see the proof of [27, Theorem 1.3.3(c), (d)]). With embedding (49) at hands, to derive (20) it thus suffices to prove that $(X, \mathscr{D}(A))_{\gamma_{1}, \infty} \hookrightarrow(X, \mathscr{D}(A))_{\gamma_{2}, 1}, 0<\gamma_{2}<\gamma_{1}<1$ (see the proof of [27, Theorem 1.3.3(e)] taking there $\left(A_{0}\right.$, $\left.A_{1}, \theta, \widetilde{\theta}\right)=\left(\mathscr{D}(A), X, 1-\gamma_{1}, 1-\gamma_{2}\right)$ and using $(\mathscr{D}(A), X)_{1-\gamma, p}=$ $\left.(X, \mathscr{D}(A))_{\gamma, p}\right)$. If we try to repeat the proof of (49) for the spaces $X_{A}^{\gamma, p}$, we will be faced with two problems. The first is that we do not know if the function $g(\xi)=\left\|A^{\circ}(\xi I-A)^{-1} x\right\|_{X}$, $\xi \in(0, \infty), x \in X$, is monotone decreasing, which would allow us to prove $X_{A}^{\gamma, p} \hookrightarrow X_{A}^{\gamma, \infty}, \gamma \in(0,1), p \in[1, \infty)$. For if $g(\xi)$ was monotone decreasing, then for every $\xi \in(0, \infty)$ and $x \in X_{A}^{\gamma, p}, \gamma \in(0,1), p \in[1, \infty)$, we would find

$$
\begin{aligned}
\xi^{\gamma} g(\xi) & =c_{9}\left(\int_{0}^{\xi} \mu^{\gamma p} \frac{\mathrm{d} \mu}{\mu}\right)^{1 / p} g(\xi) \\
& \leq c_{9}\left(\int_{0}^{\xi}\left[\mu^{\gamma} g(\mu)\right]^{p} \frac{\mathrm{d} \mu}{\mu}\right)^{1 / p} \leq c_{9}[x]_{X_{A}^{\gamma, p}},
\end{aligned}
$$

where $c_{9}=(\gamma p)^{-1 / p}$. Taking the supremum with respect to $\xi \in(0, \infty)$ in the latter inequality, we would get $[x]_{X_{A}^{\gamma, \infty}} \leq$ $c_{9}[x]_{X_{A}^{\gamma, p}}$, proving $X_{A}^{\gamma, p} \hookrightarrow X_{A}^{\gamma, \infty}, \gamma \in(0,1), p \in[1, \infty)$. The second problem is that the function $\xi^{\gamma} g(\xi)$ is not necessarily bounded for $x \in X_{A}^{\gamma, p}, \gamma \in(0,1), p \in[1, \infty)$, precluding us to prove $X_{A}^{\gamma, q} \hookrightarrow X_{A}^{\gamma, p}, \gamma \in(0,1), q \in[1, p)$. Indeed, from (35) we can only find $\xi^{\gamma} g(\xi) \leq c_{3} \xi^{\gamma}(\xi+1)^{1-\beta-\gamma}\|x\|_{X_{A}^{\gamma, p}}$, and when $\beta<1$, the right-hand side of this inequality goes to infinity as $\xi$ goes to infinity. On the contrary, if $\xi^{\gamma} g(\xi)$ were bounded, then for every $1 \leq q<p<\infty$ we would obtain

$$
\begin{aligned}
{[x]_{X_{A}^{\gamma, p}}^{p} } & =\int_{0}^{\infty}\left[\xi^{\gamma} g(\xi)\right]^{p} \frac{\mathrm{d} \xi}{\xi} \\
& \leq\left(\sup _{\xi \in(0, \infty)} \xi^{\gamma} g(\xi)\right)^{p-q} \int_{0}^{\infty}\left[\xi^{\gamma} g(\xi)\right]^{q} \frac{\mathrm{d} \xi}{\xi} \\
& =[x]_{X_{A}^{\gamma, \infty}}^{p-q}[x]_{X_{A}^{\gamma, p}}^{p}
\end{aligned}
$$

If now in addition $g(\xi)$ were also monotone decreasing, in order that $[x]_{X_{A}^{\gamma, \infty}} \leq c_{9}[x]_{X_{A}^{\gamma, q}}$, from the latter inequality we would get $[x]_{X_{A}^{\gamma, p}} \leq\left(c_{9}\right)^{(p-q) / p}[x]_{X_{A}^{\gamma, q}}$, completing the proof of $X_{A}^{\gamma, q} \hookrightarrow X_{A}^{\gamma, p}, \gamma \in(0,1), 1 \leq q<p<\infty$. Due to the former computations, we can thus conclude that in the case $\beta<1$ the quoted problems are the main obstacles which prevent us to extend (49) and, as its consequence, (20) to the spaces $X_{A}^{\gamma, p}$.

Remark 5. Let $0<\gamma_{2}<\gamma_{1}<1$ be fixed and for every $p \in$ $[1, \infty]$ and let us set $A_{p}=X_{A}^{\gamma_{2}, p}$ and $B_{p}=X_{A}^{\gamma_{1}, p}$. We thus have the two families of sets $\mathscr{A}=\left\{A_{p}\right\}_{p \in[1, \infty]}$ and $\mathscr{B}=\left\{B_{p}\right\}_{p \in[1, \infty]}$. Let first $\beta=1$. In this case, since $(X, \mathscr{D}(A))_{\gamma, p} \cong X_{A}^{\gamma, p}$, from (20) we deduce that the sets $A_{p}$ and $B_{p}$ are related by the following inclusions in which $1<q_{1}<q_{2}<\infty$ :

$$
B_{1} \subseteq B_{q_{1}} \subseteq B_{q_{2}} \subseteq B_{\infty} \subseteq A_{1} \subseteq A_{q_{1}} \subseteq A_{q_{2}} \subseteq A_{\infty}
$$

Now let $\beta<1$. As observed in Remark 4, in this case the embedding $X_{A}^{\gamma, q} \hookrightarrow X_{A}^{\gamma, p}, 1 \leq q<p \leq \infty$, may be not 
satisfied and the chain of inclusions (52) could not take place. However, (38) and (39) hold true and for every $p \in[1, \infty]$, and we have $B_{p} \subseteq A_{p}$ and $B_{\infty} \subseteq A_{p}$.

We have already pointed out that $\left\{\mathrm{e}^{t A}\right\}_{t \geq 0}$ may be not strongly continuous in the $X$-norm on $\overline{\mathscr{D}(A)}$. On the contrary, the following result (cf. [24, Proposition 5.2] for the proof) shows that the things are finer on $(X, \mathscr{D}(A))_{\gamma, p}$ and $X_{A}^{\gamma, p}$. Later, we will need this fact.

Proposition 6. Let $A$ be as in Proposition 2. If $\gamma \in(1-\beta, 1)$; then $\left\{e^{t A}\right\}_{t \geq 0}$ is strongly continuous in the X-norm on $Y_{\gamma}^{p} \in$ $\left\{(X, \mathscr{D}(A))_{\gamma, p}, X_{A}^{\gamma, p}\right\}$ for every $p \in[1, \infty]$.

We conclude the section listing some estimates for the operators $\left[(-A)^{\theta}\right]^{\circ} \mathrm{e}^{t A}$ defined by (11) with respect to the spaces $(X, \mathscr{D}(A))_{\gamma, p}$ and $X_{A}^{\gamma, p}$. First, in [19, Lemma 3.1] it is shown that $\left[(-A)^{\theta}\right]^{0} \mathrm{e}^{t A} x \in \mathscr{D}(A)$ for every $x \in X$ and that the estimate $\left\|\left[(-A)^{\theta}\right]^{\circ} \mathrm{e}^{t A} x\right\|_{\mathscr{D}(A)} \leq\left\|\left[(-A)^{\theta+1}\right]^{\circ} \mathrm{e}^{t A} x\right\|_{X}$ is satisfied. Hence, using (14), we get

$$
\begin{array}{r}
\left\|\left[(-A)^{\theta}\right]^{\circ} \mathrm{e}^{t A}\right\|_{\mathscr{L}(X ; \mathscr{D}(A))} \leq{\widetilde{c_{\alpha, \beta, \theta+1}}}^{(\beta-\Re \mathrm{e} \theta-2) / \alpha}, \\
\Re \mathrm{Re} \theta \geq 0, \quad t>0 .
\end{array}
$$

Combining (14) and (53) with (25) and letting $c_{10}=c_{0}$ $\left(c_{\alpha, \beta, \theta}\right)^{1-\gamma}\left(c_{\alpha, \beta, \theta+1}\right)^{\gamma}$, it thus follows (cf. [19, Proposition 3.1]) that for every $\gamma \in(0,1)$ and $p \in[1, \infty]$ the following estimate holds:

$$
\begin{aligned}
\left\|\left[(-A)^{\theta}\right]^{\circ} \mathrm{e}^{t A}\right\|_{\mathscr{L}\left(X ;(X, \mathscr{D}(A))_{\gamma, p}\right)} \leq & c_{10} t^{(\beta-\gamma-\Re \mathrm{e} \theta-1) / \alpha}, \\
& \Re \mathrm{Re} \theta \geq 0, \quad t>0 .
\end{aligned}
$$

Remark 7. We stress that if $\beta<1$, then we can not derive an estimate for the $\mathscr{L}\left(X ; X_{A}^{\gamma, p}\right)$-norm of $\left[(-A)^{\theta}\right]^{\circ} \mathrm{e}^{t A}$ simply by replacing $(X, \mathscr{D}(A))_{\gamma, p}$ with $X_{A}^{\gamma, p}$ in (54). This is for two reasons. First, when $\gamma \in[\beta, 1)$, we are not assured that $\left[(-A)^{\theta}\right]^{\circ}$ $\mathrm{e}^{t A} x \in X_{A}^{\gamma, p}$ for every $x \in X$. For if $\gamma \in[\beta, 1)$, then the space $X_{A}^{\gamma, p}$ may be smaller than the domain $\mathscr{D}(A)$ to which $\left[(-A)^{\theta}\right]^{\circ} e^{t A} x$ belongs by virtue of $[19$, Lemma 3.1]. The second reason is that, even limiting to $\gamma \in(0, \beta)$ in order that $\mathscr{D}(A) \hookrightarrow X_{A}^{\gamma, p}$, from (31) we only get $\|\left[(-A)^{\theta}\right]^{\circ} \mathrm{e}^{t A}$ $x\left\|_{(X, \mathscr{D}(A))_{\gamma, p}} \leq 2\right\|\left[(-A)^{\theta}\right]^{\circ} \mathrm{e}^{t A} x \|_{X_{A}^{\gamma, p}, x \in X \text {, and we do not }}$ know if the right-hand side can be bounded from above by some constant times $t^{(\beta-\gamma-\Re e \theta-1) / \alpha}\|x\|_{X}$. Of course, we can employ (32), but in this way all that we can reach is the estimate

$$
\begin{aligned}
\left\|\left[(-A)^{\theta}\right]^{\circ} \mathrm{e}^{t A}\right\|_{\mathscr{L}\left(X ; X_{A}^{\gamma+\beta-1, p}\right)} \leq & c_{11} t^{(\beta-\gamma-\Re \mathrm{e} \theta-1) / \alpha}, \\
& \mathfrak{R e} \theta \geq 0, \quad t>0,
\end{aligned}
$$

where $c_{11}=c_{2} c_{10}, \gamma \in(1-\beta, 1)$ and $p \in[1, \infty]$. Letting $\delta=\gamma+\beta-1$, (55) can be rewritten equivalently as

$$
\begin{array}{r}
\left\|\left[(-A)^{\theta}\right]^{\circ} \mathrm{e}^{t A}\right\|_{\mathscr{L}\left(X ; X_{A}^{\delta, p}\right)} \leq c_{11} t^{(2 \beta-\delta-\Re \mathrm{e} \theta-2) / \alpha}, \\
\\
\mathfrak{R e} \theta \geq 0, \quad t>0,
\end{array}
$$

where $\delta \in(0, \beta)$ and $p \in[1, \infty]$. When $\beta<1$, there are good motivations to believe that estimate (56) is not the best one. In fact, for instance, when $(\theta, p)=(0, \infty),(56)$ leads us to an estimate which is rougher than the estimate

$$
\left\|\mathrm{e}^{t A}\right\|_{\mathscr{L}\left(X ; X_{A}^{\delta, \infty}\right)} \leq c_{12} t^{(\beta-\delta-1) / \alpha}, \quad \delta \in(0,1), t>0,
$$

as shown in [2, Proposition 3.2], with $c_{12}$ being a positive constant depending on $\alpha, \beta$, and $\delta$. Also, (57) ensures that $\mathrm{e}^{t A} x, x \in X$, belongs to $X_{A}^{\delta, \infty}$ for every $\delta \in(0,1)$ and not only for $\delta \in(0, \beta)$ as (56) suggests. Furthermore, due to (31), estimate (57) yields $(54)$ with $(\theta, \gamma, p)=(0, \delta, \infty)$. This leads us to believe that (57) can be improved and that estimate (54) holds the same if $X_{A}^{\gamma, \infty}$ is taken in place of $(X, \mathscr{D}(A))_{\gamma, \infty}$.

Now let $Y_{\gamma}^{p} \in\left\{(X, \mathscr{D}(A))_{\gamma, p}, X_{A}^{\gamma, p}\right\}, \gamma \in(0,1), p \in[1, \infty]$. As far as the estimates for the $\mathscr{L}\left(Y_{\gamma}^{p} ; X\right)$-norm of operators $\left[(-A)^{\theta}\right]^{\circ} \mathrm{e}^{t A}$ are concerned, instead, at the moment only the following estimates for the case $\theta=1$ are available (cf. [24, Lemma 5.1]):

$$
\begin{gathered}
\left\|\left[(-A)^{1}\right]^{\circ} \mathrm{e}^{t A}\right\|_{\mathscr{L}\left(Y_{\gamma}^{p} ; X\right)} \leq c_{13} t^{(\beta+\gamma-2) / \alpha}, \\
t>0, \quad \gamma \in(0,1), \quad p \in[1, \infty],
\end{gathered}
$$

with $c_{13}$ being a positive constant depending on $\alpha, \beta, \gamma$, and $p$. Estimates (58) are successfully applied in [24, Corollary 5.4] to prove that if $\alpha+\beta>1$, then the map $t \rightarrow \mathrm{e}^{t A}$ is Hölder continuous from $[0, \infty)$ to $\mathscr{L}\left(Y_{\gamma}^{p} ; X\right), \gamma \in(2-\alpha-\beta, 1)$, $p \in[1, \infty]$, with Hölder exponent $\sigma=(\alpha+\beta+\gamma-2) / \alpha$. In Section 3 we will extend (58), proving some estimates for the $\mathscr{L}\left(Y_{\gamma}^{p} ; X\right)$-norm of $\left[(-A)^{\theta}\right]^{\circ} \mathrm{e}^{t A}, \mathfrak{R e} \theta \geq 1$, which reduce to $(58)$ in the case $\theta=1$.

Remark 8. Observe that an estimate for the norm $\|\left[(-A)^{\theta}\right]^{\circ}$ $\mathrm{e}^{t A} \|_{\mathscr{L}\left(Y_{\gamma}^{p} ; X\right)}, \mathfrak{R e} \theta \geq 1, t>0, Y_{\gamma}^{p} \in\left\{(X, \mathscr{D}(A))_{\gamma, p}, X_{A}^{\gamma, p}\right\}, \gamma \in$ $(0,1), p \in[1, \infty]$, can be obtained combining (14), (15), and (58). Indeed, using (15), for every $\mathfrak{R e} \theta \geq 1, t>0$ and $x \in Y_{\gamma}^{p}$, we have

$$
\begin{aligned}
& \left\|\left[(-A)^{\theta}\right]^{\circ} \mathrm{e}^{t A} x\right\|_{X} \\
& \quad=\left\|\left[(-A)^{\theta-1}\right]^{\circ} \mathrm{e}^{(t / 2) A}\left[(-A)^{1}\right]^{\circ} \mathrm{e}^{(t / 2) A} x\right\|_{X} \\
& \leq\left\|\left[(-A)^{\theta-1}\right]^{\circ} \mathrm{e}^{(t / 2) A}\right\|_{\mathscr{L}(X)}\left\|\left[(-A)^{1}\right]^{\circ} \mathrm{e}^{(t / 2) A} x\right\|_{X} .
\end{aligned}
$$

Therefore, due to (14) and (58), from (59) we deduce that

$$
\left\|\left[(-A)^{\theta}\right]^{\circ} \mathrm{e}^{t A}\right\|_{\mathscr{L}\left(Y_{\gamma}^{p} ; X\right)} \leq c_{14} t^{(2 \beta+\gamma-\Re \mathrm{e} \theta-2) / \alpha},
$$

$$
\Re \text { e } \theta \geq 1, \quad t>0 \text {, }
$$


where $\gamma \in(0,1), p \in[1, \infty]$ and $c_{14}=2^{(2+\Re \mathrm{e} \theta-\gamma-2 \beta) / \alpha}$ $\widetilde{c}_{\alpha, \beta, \theta-1} c_{13}$. As we will see in the next section estimate $(60)$ is not optimal, in the sense that the negative exponent $(2 \beta+$ $\gamma-\mathfrak{R e} \theta-2) / \alpha$ can be refined; of course, unless $\beta=1$. The main reason to believe that (60) can be improved is that its derivation consists of two steps: the first in which $\left[(-A)^{\theta}\right]^{\circ} \mathrm{e}^{t A}$ is decomposed with the help of (15), and the second in which (60) is obtained combining estimates of very different nature, such as (14) and (58). It is thus to be expected that in this double step derivation some regularity goes missing and that a better result can be reached analyzing more detailedly $\left[(-A)^{\theta}\right]^{\circ} \mathrm{e}^{t A} x$ for $x \in Y_{\gamma}^{p}$.

\section{Behaviour of $\left[(-A)^{\theta}\right]^{\circ} \mathrm{e}^{t A}$ in $(X, \mathscr{D}(A))_{\gamma, p}$ and $X_{A}^{\gamma, p}$}

According to Remark 7 we begin by improving (54), showing that the same estimate holds with $(X, \mathscr{D}(A))_{\gamma, p}$ being replaced by $X_{A}^{\gamma, \infty}$ if $p=\infty$ and by $X_{A}^{\beta \gamma, p}$ if $p \in[1, \infty)$. Throughout this and the next section, $A$ will be an $\mathrm{m}$. l. operator in $X$ having nonempty domain $\mathscr{D}(A)$ and satisfying the resolvent condition $(\mathrm{H} 1)$ of Section 2.

Proposition 9. Let $\mathfrak{R e} \theta \geq 0, \gamma \in(0,1)$ and let $p \in[1, \infty]$. Then, there exist positive constants $c_{j}, j=15,16$, depending on $\alpha, \beta, \gamma, \theta$, and $p$ such that

$$
\begin{array}{r}
\left\|\left[(-A)^{\theta}\right]^{\circ} e^{t A}\right\|_{\mathscr{L}\left(X ; X_{A}^{\gamma, \infty}\right)} \leq c_{15} t^{(\beta-\gamma-\Re \mathrm{e} \theta-1) / \alpha}, \\
t>0, \quad p=\infty, \\
\left\|\left[(-A)^{\theta}\right]^{\circ} e^{t A}\right\|_{\mathscr{L}\left(X ; X_{A}^{\beta \gamma, p}\right)} \leq c_{16} t^{(\beta-\gamma-\Re \mathrm{e} \theta-1) / \alpha}, \\
t>0, \quad p \in[1, \infty) .
\end{array}
$$

Proof. If $\beta=1$, then $(X, \mathscr{D}(A))_{\gamma, p} \cong X_{A}^{\gamma, p}$ and (61) and (62) with $c_{j}=c_{2} c_{10}, j=15,16$, follow by taking $\beta=1$ in (32) and (54). Therefore, without the loss of generality, we assume that $\beta \in(0, \alpha]$ is such that $\beta<\alpha$ if $\alpha=1$. Let $\theta \in \mathbf{C}, \mathfrak{R e} \theta \geq 0$, $\gamma \in(0,1)$, and $p \in[1, \infty)$ be fixed and let $x$ be an arbitrary element of $X$. Then, for every $t>0$ we have

$$
\begin{aligned}
& \left\|\left[(-A)^{\theta}\right]^{\circ} \mathrm{e}^{t A} x\right\|_{X_{A}^{\gamma, \infty}} \\
& =\left\|\left[(-A)^{\theta}\right]^{\circ} \mathrm{e}^{t A} x\right\|_{X}+\left\|\xi^{\gamma} A^{\circ}(\xi I-A)^{-1}\left[(-A)^{\theta}\right]^{\circ} \mathrm{e}^{t A} x\right\|_{L_{\infty}^{*}(X)},
\end{aligned}
$$

$$
\begin{aligned}
& \left\|\left[(-A)^{\theta}\right]^{\circ} \mathrm{e}^{t A} x\right\|_{X_{A}^{\beta \gamma, p}} \\
& =\left\|\left[(-A)^{\theta}\right]^{\circ} \mathrm{e}^{t A} x\right\|_{X}+\left\|\xi^{\beta \gamma} A^{\circ}(\xi I-A)^{-1}\left[(-A)^{\theta}\right]^{\circ} \mathrm{e}^{t A} x\right\|_{L_{p}^{*}(X)} .
\end{aligned}
$$

Of course, from estimate (54) we find

$$
\begin{array}{r}
\left\|\left[(-A)^{\theta}\right]^{\circ} \mathrm{e}^{t A} x\right\|_{X} \leq c_{\gamma, p} c_{10}\|x\|_{X} t^{(\beta-\gamma-\Re \mathrm{e} \theta-1) / \alpha}, \\
t>0,
\end{array}
$$

with $c_{\gamma, p}$ being such that $\|y\|_{X} \leq c_{\gamma, p}\|y\|_{(X, \mathscr{D}(A))_{\gamma, p}}, y \in(X$, $\mathscr{D}(A))_{\gamma, p}, p \in[1, \infty]$. It thus suffices to investigate only the second terms on the right-hand side of (63) and (64). We begin by proving (61). First, using the second identity in (6), for every $\xi \in(0, \infty)$ we get

$$
\begin{aligned}
\xi^{\gamma} A^{\circ}(\xi I-A)^{-1}\left[(-A)^{\theta}\right]^{\circ} \mathrm{e}^{t A} x & \frac{1}{2 \pi i} \int_{\Gamma} \xi^{\gamma}(-\lambda)^{\theta} \mathrm{e}^{t \lambda} A^{\circ}(\xi I-A)^{-1}(\lambda I-A)^{-1} x \mathrm{~d} \lambda \\
= & \xi^{\gamma}\left[\frac{1}{2 \pi i} \int_{\Gamma}(-\lambda)^{\theta} \mathrm{e}^{t \lambda}(\lambda-\xi)^{-1} \mathrm{~d} \lambda\right] A^{\circ}(\xi I-A)^{-1} x \\
& -\frac{1}{2 \pi i} \int_{\Gamma} \xi^{\gamma}(-\lambda)^{\theta} \mathrm{e}^{t \lambda}(\lambda-\xi)^{-1} A^{\circ}(\lambda I-A)^{-1} x \mathrm{~d} \lambda \\
= & -\frac{1}{2 \pi i} \int_{\Gamma} \xi^{\gamma}(-\lambda)^{\theta} \mathrm{e}^{t \lambda}(\lambda-\xi)^{-1}\left[\lambda(\lambda I-A)^{-1}-I\right] x \mathrm{~d} \lambda \\
= & \frac{1}{2 \pi i} \int_{\Gamma} \xi^{\gamma}(-\lambda)^{\theta+1} \mathrm{e}^{t \lambda}(\lambda-\xi)^{-1}(\lambda I-A)^{-1} x \mathrm{~d} \lambda .
\end{aligned}
$$

Here we have used twice the equality $\int_{\Gamma}(-\lambda)^{\theta} \mathrm{e}^{t \lambda}(\lambda-\xi)^{-1} \mathrm{~d} \lambda=$ $0, \xi \in(0, \infty)$, which follows from Cauchy's formula after having enclosed $\Gamma$ on the left with an arc of the circle $\{z \in$ C : $|z+c|=R\}, R>0$, and letting $R$ to infinity. From (66), using $\left\|(\lambda I-A)^{-1}\right\|_{\mathscr{L}(X)} \leq C(|\lambda|+1)^{-\beta} \leq C|\lambda|^{-\beta}, \lambda \in \Sigma_{\alpha}$, it follows that

$$
\begin{aligned}
&\left\|\xi^{\gamma} A^{\circ}(\xi I-A)^{-1}\left[(-A)^{\theta}\right]^{\circ} \mathrm{e}^{t A} x\right\|_{X} \\
& \leq C(2 \pi)^{-1}\|x\|_{X} \\
& \times \int_{\Gamma} \xi^{\gamma}|\lambda|^{1+\Re \mathrm{e} \theta-\beta} \mathrm{e}^{-\Im \mathrm{m} \theta \arg (-\lambda)} \mathrm{e}^{t \Re \mathrm{e} \lambda}|\lambda-\xi|^{-1}|\mathrm{~d} \lambda| \\
& \leq C(2 \pi)^{-1} \mathrm{e}^{(\pi / 2)|\Im \mathrm{m} \theta|}\|x\|_{X} \\
& \times \int_{\Gamma}\left(\frac{\xi}{|\lambda|}\right)^{\gamma}|\lambda|^{\gamma+\Re \mathrm{e} \theta-\beta} \mathrm{e}^{t \Re \mathrm{e} \lambda}\left|1-\left(\frac{\xi}{\lambda}\right)\right|^{-1}|\mathrm{~d} \lambda|
\end{aligned}
$$

Now, since $\mathfrak{R e} \lambda \leq-c<0$ for every $\lambda \in \Gamma$ and since $\xi \in(0, \infty)$, we have

$$
\begin{aligned}
\left|1-\left(\frac{\xi}{\lambda}\right)\right| & =\left|1-\left(\frac{\xi \bar{\lambda}}{|\lambda|^{2}}\right)\right| \\
& =\left[1+\left(\frac{\xi}{|\lambda|}\right)^{2}-\frac{2 \xi \Re \mathrm{e} \lambda}{|\lambda|^{2}}\right]^{1 / 2} \\
& \geq\left[1+\left(\frac{\xi}{|\lambda|}\right)^{2}\right]^{1 / 2} .
\end{aligned}
$$


Therefore, for every $\lambda \in \Gamma$ and $\xi \in(0, \infty)$ the following inequality holds:

$$
\begin{aligned}
\left(\frac{\xi}{|\lambda|}\right)^{\gamma}\left|1-\left(\frac{\xi}{\lambda}\right)\right|^{-1} & \leq\left(\frac{\xi}{|\lambda|}\right)^{\gamma}\left[1+\left(\frac{\xi}{|\lambda|}\right)^{2}\right]^{-1 / 2} \\
& \leq \gamma^{\gamma / 2}(1-\gamma)^{(1-\gamma) / 2}=: c_{\gamma}
\end{aligned}
$$

where we have used the fact that the function $f(s)=s^{\gamma}(1+$ $\left.s^{2}\right)^{-1 / 2}, s \geq 0, \gamma \in(0,1)$, attains its maximum value $c_{\gamma}$ at the point $s_{\gamma}=\gamma^{1 / 2}(1-\gamma)^{-1 / 2}$. Coming back to (67) and setting $c_{17}=C(2 \pi)^{-1} \mathrm{e}^{(\pi / 2)|\Im \mathrm{m} \theta|} c_{\gamma}$, we thus find (here we use also that on $\Gamma$ it holds $|\lambda| \geq c$, so that $\mathfrak{R e} \lambda=-c(|\Im \mathrm{Im} \lambda|+1)^{\alpha} \geq-c$ $\left.\left(1+c^{-1}\right)^{\alpha}|\lambda|^{\alpha}\right)$ :

$$
\begin{aligned}
& \left\|\xi^{\gamma} A^{\circ}(\xi I-A)^{-1}\left[(-A)^{\theta}\right]^{\circ} \mathrm{e}^{t A} x\right\|_{X} \\
& \quad \leq c_{17}\|x\|_{X} \int_{\Gamma}|\lambda|^{\gamma+\Re \mathrm{e} \theta-\beta} \mathrm{e}^{t \Re \mathrm{e} \lambda}|\mathrm{d} \lambda| \\
& \quad \leq c_{17}\|x\|_{X} \int_{\Gamma}|\lambda|^{\gamma+\Re \mathrm{e} \theta-\beta} \mathrm{e}^{-c\left(1+c^{-1}\right)^{\alpha} t|\lambda|^{\alpha}}|\mathrm{d} \lambda| \\
& \quad \leq 2 c_{17}\|x\|_{X} \int_{0}^{\infty} \mu^{\gamma+\Re \mathrm{e} \theta-\beta} \mathrm{e}^{-c_{\alpha} t \mu^{\alpha}} \mathrm{d} \mu,
\end{aligned}
$$

where $c_{\alpha}=c\left(1+c^{-1}\right)^{\alpha}$. Finally, taking the supremum with respect to $\xi \in(0, \infty)$ in $(70)$ and performing the transformation $c_{\alpha} t \mu^{\alpha}=s$ in the integral on the right, we obtain

$$
\begin{gathered}
\left\|\xi^{\gamma} A^{\circ}(\xi I-A)^{-1}\left[(-A)^{\theta}\right]^{\circ} \mathrm{e}^{t A} x\right\|_{L_{\infty}^{*}(X)} \\
\leq c_{18}\|x\|_{X} t^{(\beta-\gamma-\Re \mathrm{e} \theta-1) / \alpha},
\end{gathered}
$$

where $c_{18}=2 c_{17} \alpha^{-1} c_{\alpha}^{(\beta-\gamma-\Re e \theta-1) / \alpha} E((\gamma+\mathfrak{R e} \theta+1-\beta) / \alpha)$, $E(\chi), \chi>0$, being the Euler gamma function $\int_{0}^{\infty} s^{\chi-1} \mathrm{e}^{-s} \mathrm{~d} s$. Then, summing up (65) and (71), from (63) it follows that

$$
\begin{array}{r}
\left\|\left[(-A)^{\theta}\right]^{\circ} \mathrm{e}^{t A} x\right\|_{X_{A}^{\gamma, \infty}} \leq\left(c_{\gamma, \infty} c_{10}+c_{18}\right)\|x\|_{X} t^{(\beta-\gamma-\Re \mathrm{e} \theta-1) / \alpha}, \\
\Re \mathrm{Re} \theta \geq 0, \quad t>0 .
\end{array}
$$

Since $x \in X$ was arbitrary, this completes the proof of (61) with $c_{15}=c_{\gamma, \infty} c_{10}+c_{18}$. Let us now prove (62). For every $p \in$ $[1, \infty)$ we write

$$
\left\|\xi^{\beta \gamma} A^{\circ}(\xi I-A)^{-1}\left[(-A)^{\theta}\right]^{\circ} \mathrm{e}^{t A} x\right\|_{L_{p}^{*}(X)}^{p}=I_{1}+I_{2},
$$

where $I_{j}=\int_{a_{j}}^{b_{j}}\left\|\xi^{\beta \gamma} A^{\circ}(\xi I-A)^{-1}\left[(-A)^{\theta}\right]^{\circ} \mathrm{e}^{t A} x\right\|_{X}^{p}(\mathrm{~d} \xi / \xi), j=$ $1,2,\left(a_{1}, b_{1}, a_{2}, b_{2}\right)=(0,1,1, \infty)$. First, (35) with $Y_{\gamma}^{p}=(X$, $\mathscr{D}(A))_{\gamma, p}$ yields

$$
I_{1} \leq\left\|\left[(-A)^{\theta}\right]^{\circ} \mathrm{e}^{t A} x\right\|_{(X, \mathscr{D}(A))_{\gamma, p}}^{p} \int_{0}^{1} \xi^{\beta \gamma p-1}\left[c_{3}(\xi+1)^{1-\beta-\gamma}\right]^{p} \mathrm{~d} \xi .
$$

Therefore, since $(\xi+1)^{1-\beta-\gamma} \leq c_{\beta, \gamma}$ for every $\xi \in(0,1]$, where $c_{\beta, \gamma}=2^{1-\beta-\gamma}$ or $c_{\beta, \gamma}=1$ according that $\gamma \in(0,1-\beta)$ or $\gamma \in[1-\beta, 1)$, from $(54)$, we deduce that

$$
\begin{aligned}
I_{1} & \leq\left[c_{\beta, \gamma} c_{3}\right]^{p}\left\|\left[(-A)^{\theta}\right]^{\circ} \mathrm{e}^{t A} x\right\|_{(X, \mathscr{D}(A))_{\gamma, p}}^{p} \int_{0}^{1} \xi^{\beta \gamma p-1} \mathrm{~d} \xi \\
& =\left[c_{19}\|x\|_{X} t^{(\beta-\gamma-\Re \mathrm{R} \theta-1) / \alpha}\right]^{p},
\end{aligned}
$$

with $c_{19}=c_{\beta, \gamma} c_{3} c_{10}(\beta \gamma p)^{-1 / p}$. As far as $I_{2}$ is concerned, exploiting (71) and recalling that we have assumed $\beta<1$, we obtain

$$
\begin{aligned}
I_{2} & =\int_{1}^{\infty} \xi^{(\beta-1) \gamma p}\left\|\xi^{\gamma} A^{\circ}(\xi I-A)^{-1}\left[(-A)^{\theta}\right]^{\circ} \mathrm{e}^{t A} x\right\|_{X}^{p} \frac{\mathrm{d} \xi}{\xi} \\
& \leq\left[c_{18}\|x\|_{X} t^{(\beta-\gamma-\Re e \theta-1) / \alpha}\right]^{p} \int_{1}^{\infty} \xi^{(\beta-1) \gamma p-1} \mathrm{~d} \xi \\
& \leq\left[c_{20}\|x\|_{X} t^{(\beta-\gamma-\Re e \theta-1) / \alpha}\right]^{p},
\end{aligned}
$$

where $c_{20}=c_{18}[(1-\beta) \gamma p]^{-1 / p}$. Summing up (73)-(76), it thus follows that

$$
\begin{gathered}
\left\|\xi^{\beta \gamma} A^{\circ}(\xi I-A)^{-1}\left[(-A)^{\theta}\right]^{\circ} \mathrm{e}^{t A} x\right\|_{L_{p}^{*}(X)} \\
\leq c_{21}\|x\|_{X} t^{(\beta-\gamma-\Re \mathrm{e} \theta-1) / \alpha},
\end{gathered}
$$

where $c_{21}=\left[\left(c_{19}\right)^{p}+\left(c_{20}\right)^{p}\right]^{1 / p}$. Finally, (65) and (77) lead us to

$$
\begin{array}{r}
\left\|\left[(-A)^{\theta}\right]^{\circ} \mathrm{e}^{t A} x\right\|_{X_{A}^{\beta \gamma, p}} \leq\left(c_{\gamma, p} c_{10}+c_{21}\right)\|x\|_{X} t^{(\beta-\gamma-\Re \mathrm{e} \theta-1) / \alpha}, \\
\mathfrak{R e} \theta \geq 0, \quad t>0 .
\end{array}
$$

Since $x \in X$ was arbitrary, this completes the proof of (62) with $c_{16}=c_{\gamma, p} c_{10}+c_{21}$.

Remark 10. If $\theta=0$, then (61) is precisely the estimate (57). In this sense our result improves [2] and shows that (54) holds the same with $(X, \mathscr{D}(A))_{\gamma, p}$ being replaced with $X_{A}^{\gamma, \infty}$ if $p=$ $\infty$ and $X_{A}^{\beta \gamma, p}$ and if $p \in[1, \infty)$. Also, when $\beta<1,(61)$ and (62) are in two aspects better than the estimate (55) deduced from (54) with the help of (32). First, here we do not need to restrict $\gamma$ to $(1-\beta, 1)$. Further, despite limiting $\gamma$ to $(1-\beta, 1)$, (61) and (62) show that $\left[(-A)^{\theta}\right]^{\circ} \mathrm{e}^{t A} x, \mathfrak{R e} \theta \geq 0, t>0, x \in X$, enjoys more regularity than that predicted by (55). For, since when $\beta<1$ it holds $0<\gamma+\beta-1<\beta \gamma<\gamma$, from (38) and (39) it follows $X_{A}^{\gamma, \infty} \hookrightarrow X_{A}^{\beta \gamma, p} \hookrightarrow X_{A}^{\gamma+\beta-1, p}, p \in[1, \infty]$.

Remark 11. We recall that when $\beta<1$ the spaces $X_{A}^{\sigma, p}, \sigma \in$ $(0,1), p \in[1, \infty]$, are intermediate spaces between $X$ and $\mathscr{D}(A)$ for $\sigma \in(0, \beta)$, but they may be contained in $\mathscr{D}(A)$ for $\sigma \in[\beta, 1)$. Therefore, whereas (61) is satisfied for spaces $X_{A}^{\sigma, \infty}$ eventually smaller than $\mathscr{D}(A)$, for (62) to hold we have to consider only spaces $X_{A}^{\sigma, p}, p \in[1, \infty)$, bigger than $\mathscr{D}(A)$. In fact, letting $\sigma=\beta \gamma$, we have $\sigma \in(0, \beta)$ for every $\gamma \in(0,1)$. 

(58).

In accordance with Remark 8 we now improve estimate

Proposition 12. Let $R \mathrm{e} \theta \geq 1, \gamma \in(0,1), p \in[1, \infty]$ and let $Y_{\gamma}^{p} \in\left\{(X, \mathscr{D}(A))_{\gamma, p}, X_{A}^{\gamma, p}\right\}$. Then, there exists a positive constant $c_{22}$ depending on $\alpha, \beta, \gamma, \theta$, and $p$ such that

$$
\left\|\left[(-A)^{\theta}\right]^{\circ} e^{t A}\right\|_{\mathscr{L}\left(Y_{\gamma}^{p} ; X\right)} \leq c_{22} t^{(\beta+\gamma-\Re \mathrm{e} \theta-1) / \alpha}, \quad t>0 .
$$

Proof. First, using the identity $A^{\circ}(z I-A)^{-1}=z(z I-A)^{-1}-I$, $z \in \Sigma_{\alpha}$, for every $x \in X$, we rewrite $\left[(-A)^{\theta}\right]^{0} \mathrm{e}^{t A} x, \mathfrak{R e} \theta \geq 0$, in the following way:

$$
\begin{aligned}
& {\left[(-A)^{\theta}\right]^{\circ} \mathrm{e}^{t A} x} \\
& \quad=-\frac{1}{2 \pi i} \int_{\Gamma}(-\lambda)^{\theta-1} \mathrm{e}^{t \lambda} \lambda(\lambda I-A)^{-1} x \mathrm{~d} \lambda \\
& \quad=-\frac{1}{2 \pi i} \int_{\Gamma}(-\lambda)^{\theta-1} \mathrm{e}^{t \lambda}\left[A^{\circ}(\lambda I-A)^{-1} x+I\right] x \mathrm{~d} \lambda \\
& \quad=-\frac{1}{2 \pi i} \int_{\Gamma}(-\lambda)^{\theta-1} \mathrm{e}^{t \lambda} A^{\circ}(\lambda I-A)^{-1} x \mathrm{~d} \lambda, \quad t>0 .
\end{aligned}
$$

Here we have used $\int_{\Gamma}(-\lambda)^{\theta-1} \mathrm{e}^{t \lambda} \mathrm{d} \lambda=0$, which follows from the Cauchy formula applied to $(-\lambda)^{\theta} \mathrm{e}^{t \lambda}$ after having enclosed $\Gamma$ on the left with an arc of the circle $\{z \in \mathbf{C}:|z+c|=R\}$, $R>0$, and letting $R$ to infinity. Let now $\theta \in \mathbf{C}, \mathfrak{R e} \theta \geq 1$, $\gamma \in(0,1)$, and $p \in[1, \infty]$ be fixed and let $x$ be an arbitrary element of $Y_{\gamma}^{p}$. From (35) it then follows that

$$
\begin{aligned}
& \left\|\left[(-A)^{\theta}\right]^{0} \mathrm{e}^{t A} x\right\|_{X} \\
& \quad \leq \mathrm{c}_{23}\|x\|_{Y_{\gamma}^{p}} \int_{\Gamma}|\lambda|^{\Re \mathrm{R} \theta-1} \mathrm{e}^{t \mathfrak{R e} \lambda}(|\lambda|+1)^{1-\beta-\gamma}|\mathrm{d} \lambda|, \quad t>0,
\end{aligned}
$$

where $c_{23}=(2 \pi)^{-1} \mathrm{e}^{(\pi / 2)|\Im \mathrm{m} \theta|} c_{3}$. Now, recalling that $|\lambda| \geq c>$ 0 for every $\lambda \in \Gamma$, we have $|\lambda| \leq|\lambda|+1 \leq\left(1+c^{-1}\right)|\lambda|, \lambda \in \Gamma$. As a consequence, the following inequality holds:

$$
(|\lambda|+1)^{1-\beta-\gamma} \leq \widetilde{c}_{\beta, \gamma}|\lambda|^{1-\beta-\gamma}, \quad \forall \lambda \in \Gamma,
$$

where $\widetilde{c}_{\beta, \gamma}=\left(1+c^{-1}\right)^{1-\beta-\gamma}$ or $\widetilde{c}_{\beta, \gamma}=1$ according that $\gamma \in$ $(0,1-\beta]$ or $\gamma \in(1-\beta, 1)((0,1-\beta]=\emptyset$ if $\beta=1)$. Therefore, setting $c_{24}=2 \widetilde{c}_{\beta, \gamma} c_{23},(81)$ and (82) yield

$$
\begin{aligned}
& \left\|\left[(-A)^{\theta}\right]^{\circ} \mathrm{e}^{t A} x\right\|_{X} \\
& \quad \leq c_{24}\|x\|_{Y_{\gamma}^{p}} \int_{0}^{\infty} \mu^{\Re \mathrm{Re} \theta-\beta-\gamma} \mathrm{e}^{-c_{\alpha} t \mu^{\alpha}} \mathrm{d} \mu, \quad t>0,
\end{aligned}
$$

with $c_{\alpha}$ being as in (70). Finally, the transformation $c_{\alpha} t \mu^{\alpha}=s$ in the last integral leads us to the following estimate:

$$
\left\|\left[(-A)^{\theta}\right]^{\circ} \mathrm{e}^{t A} x\right\|_{X} \leq c_{25}\|x\|_{Y_{\gamma}^{p} t^{(\beta+\gamma-\Re \mathrm{e} \theta-1) / \alpha}, \quad t>0,}
$$

where $c_{25}=c_{24} \alpha^{-1} c_{\alpha}^{(\beta+\gamma-\operatorname{Re} \theta-1) / \alpha} E((\operatorname{Re} \theta+1-\beta-\gamma) / \alpha)$, $E(\chi), \chi>0$, is the Euler's gamma function. Notice that here
$\Re$ e $\theta \geq 1$ implies $\mathfrak{R e} \theta+1-\beta-\gamma \geq 2-\beta-\gamma>0$ for every $\beta \in(0,1]$ and $\gamma \in(0,1)$, so that $E((\Re \operatorname{Re} \theta+1-\beta-\gamma) / \alpha)$ makes sense. Since (84) is satisfied for every arbitrary element $x \in Y_{\gamma}^{p}$, the proof is complete with $c_{22}=c_{25}$.

Remark 13. Estimate (79) is better than (60) obtained in Remark 8 using (14), (15), and (58). In fact, for every $\beta \in$ $(0, \alpha], \alpha \in(0,1], \gamma \in(0,1)$ and $\operatorname{Re} \theta \geq 1$, the following inequality holds:

$$
\begin{aligned}
\rho_{1} & :=\frac{(2 \beta+\gamma-\Re \mathrm{e} \theta-2)}{\alpha} \\
& \leq \frac{(\beta+\gamma-\Re \mathrm{e} \theta-1)}{\alpha}:=\rho_{2}<0 .
\end{aligned}
$$

Then, $t^{\rho_{2}} \leq t^{\rho_{1}}, t \in(0,1]$, and (79) is more accurate than (60) for small values of $t$.

Estimate (79) with $\theta=1$ yields the following result which we will need in Section 5 to prove the equivalence between problem (170) and the fixed-point equation (179).

Corollary 14. Let $\alpha+\beta>1$ in (H1). Then, for every $x \in X$ the following equalities hold:

$$
A^{-1}\left(e^{t A}-I\right) x=\left(e^{t A}-I\right) A^{-1} x=\int_{0}^{t} e^{(t-s) A} x d s, \quad t \geq 0 .
$$

Proof. The assertion is obvious for $t=0$. Let $t>0$ and let $x \in X$. Commuting $A^{-1} \in \mathscr{L}(X)$ with the integral sign, from (9) and the resolvent equation, we have $A^{-1} \mathrm{e}^{t A} x=\mathrm{e}^{t A} A^{-1} x$, which proves the first equality in (86). To prove the second equality, we first write

$$
\begin{aligned}
\left(\mathrm{e}^{t A}-I\right) A^{-1} x & =\int_{0}^{t}\left[D_{r} \mathrm{e}^{r A}\right]_{r=t-s} A^{-1} x \mathrm{~d} s \\
& =-\int_{0}^{t}\left[(-A)^{1}\right]^{\circ} \mathrm{e}^{(t-s) A} A^{-1} x \mathrm{~d} s,
\end{aligned}
$$

and we show that the latter integral is convergent. Indeed, since $\alpha+\beta>1$, we may consider $A^{-1} x \in \mathscr{D}(A)$ as an element of $(X, \mathscr{D}(A))_{\gamma, p}$, where $\gamma \in(2-\alpha-\beta, 1)$ and $p \in[1, \infty]$. With this choice for $\gamma$, from (79) with $\theta=1$ and (25) we obtain (here we use also $\left\|A^{-1} x\right\|_{\mathscr{D}(A)}=\inf _{y \in A\left(A^{-1} x\right)}\|y\|_{X}=$ $\inf _{y \in\left(A A^{-1}\right) x}\|y\|_{X}=\|x\|_{\mathscr{D}\left(A A^{-1}\right)} \leq\|x\|_{X}$, due to $I \subset A A^{-1}$. Then, $\left\|A^{-1} x\right\|_{(X, \mathscr{D}(A))_{\gamma, p}} \leq c_{0}\left\|A^{-1} x\right\|_{X}^{1-\gamma}\left\|A^{-1} x\right\|_{\mathscr{D}(A)}^{\gamma} \leq c_{0}$ $\left.\left\|A^{-1}\right\|_{\mathscr{L}(X)}^{1-\gamma}\|x\|_{X}\right):$

$$
\begin{aligned}
& \left\|\int_{0}^{t}\left[(-A)^{1}\right]^{\circ} \mathrm{e}^{(t-s) A} A^{-1} x \mathrm{~d} s\right\|_{X} \\
& \quad \leq c_{22}\left\|A^{-1} x\right\|_{(X, \mathscr{D}(A))_{\gamma, p}} \int_{0}^{t}(t-s)^{(\beta+\gamma-2) / \alpha} \mathrm{d} s \\
& \quad \leq c_{22} c_{\alpha, \beta, \gamma} c_{0}\left\|A^{-1}\right\|_{\mathscr{L}(X)}^{1-\gamma}\|x\|_{X} t^{(\alpha+\beta+\gamma-2) / \alpha},
\end{aligned}
$$


where $c_{\alpha, \beta, \gamma}=\alpha(\alpha+\beta+\gamma-2)^{-1}$. We now recall that (cf. [24, formula (3.21)])

$$
\left[(-A)^{1}\right]^{\circ} \mathrm{e}^{t A}(-A)^{-\zeta}=\left[(-A)^{1-\zeta}\right]^{\circ} \mathrm{e}^{t A}, \quad \mathfrak{R e} \zeta \in(1-\beta, 1]
$$

with $(-A)^{-\zeta}$ being the negative fractional powers of $-A$ defined by (cf. [24, Section 3]) $(2 \pi i)^{-1} \int_{\Gamma}(-\lambda)^{-\zeta}(\lambda I-A)^{-1} \mathrm{~d} \lambda$, $\mathfrak{R e} \zeta>1-\beta$. To complete the proof it thus suffices to apply (89) with $\zeta=1$ to (87) and to recall that $\left[(-A)^{0}\right]^{\circ} \mathrm{e}^{t A}=\mathrm{e}^{t A}$, $t>0$. Notice that the integral on the right-hand side of (86) is convergent, too. In fact, from (14), it follows that $\left\|\int_{0}^{t} \mathrm{e}^{(t-s) A} x \mathrm{~d} s\right\|_{\mathrm{X}} \leq \widetilde{c}_{\alpha, \beta, 0}\|x\|_{X} \int_{0}^{t}(t-s)^{(\beta-1) / \alpha} \mathrm{d} s=\alpha(\alpha+\beta-$ $1)^{-1} \widetilde{c}_{\alpha, \beta, 0}\|x\|_{X} t^{(\alpha+\beta-1) / \alpha}$.

Remark 15. In particular, from (86) it follows that if $\alpha+\beta>1$, then $\int_{0}^{t} \mathrm{e}^{(t-s) A} x \mathrm{ds} \in \mathscr{D}(A)$ for every $x \in X$ and $\left(\mathrm{e}^{t A}-I\right) x \subseteq$ $A \int_{0}^{t} \mathrm{e}^{(t-s) A} x \mathrm{ds}$. This extends to $\mathrm{m}$. 1. operators satisfying (H1) the well-known result for sectorial single-valued linear operators (see, for instance, [9, Proposition 2.1.4(ii)] and [11, Proposition 1.2(ii)]).

With the help of (54) and Proposition 12, we can now derive the following interpolation estimates (90) for the operators $\left[(-A)^{\theta}\right]^{\circ} \mathrm{e}^{t A}, \mathfrak{R e} \theta \geq 1$, which are considered as operators from $(X, \mathscr{D}(A))_{\gamma, p}$ to $(X, \mathscr{D}(A))_{\delta, p}$. As we will see in the proof of Proposition 16, here the fact that the spaces $(X, \mathscr{D}(A))_{\sigma, p}$ are real interpolation spaces between $X$ and $\mathscr{D}(A)$ plays a key role. For it allows us to exploit the interpolation inequality (24) in the derivation of our estimates in the case $\gamma+\delta<1$.

Proposition 16. Let $\mathfrak{R e} \theta \geq 1, \gamma, \delta \in(0,1)$, and $p \in[1, \infty]$. Then, there exist positive constants $c_{j}, j=26,27$, depending on $\alpha, \beta, \gamma, \delta, \theta$, and $p$ such that for every $t>0$

$$
\begin{gathered}
\left\|\left[(-A)^{\theta}\right]^{\circ} e^{t A}\right\|_{\mathscr{L}\left((X, \mathscr{D}(A))_{\gamma, p} ;(X, \mathscr{D}(A))_{\delta, p}\right)} \\
\leq \begin{cases}c_{26} t^{(2 \beta+\gamma-\delta-\Re \mathrm{e} \theta-2) / \alpha}, & \gamma, \delta \in(0,1), \\
\mathcal{C}_{27} t^{(\beta+\gamma-\delta-\Re \mathrm{e} \theta-1) / \alpha}, & \text { if } \gamma+\delta<1 .\end{cases}
\end{gathered}
$$

Proof. For brevity, we will use the shortenings $Y_{\sigma}^{p}=(X$, $\mathscr{D}(A))_{\sigma, p}, \sigma \in(0,1), p \in[1, \infty]$. We begin by proving the first estimate in (90). Let $\theta \in \mathbf{C}, \mathfrak{R e} \theta \geq 1, \gamma, \delta \in(0,1)$ and $p \in[1, \infty]$ be fixed and let $x$ be an arbitrary element of $Y_{\gamma}^{p}$. Moreover, let $\zeta$ and $\zeta^{\prime}$ be two arbitrary complex numbers such that $\theta=\zeta+\zeta^{\prime}$ and whose real parts satisfy $\mathfrak{R e} \zeta \geq 0$ and $\mathfrak{R e} \zeta^{\prime} \geq 1$. From the decomposition formula (15) it then follows for every $t>0$ :

$$
\begin{aligned}
& \left\|\left[(-A)^{\theta}\right]^{\circ} \mathrm{e}^{t A} x\right\|_{Y_{\delta}^{p}} \\
& \quad=\left\|\left[(-A)^{\zeta}\right]^{\circ} \mathrm{e}^{(t / 2) A}\left[(-A)^{\zeta^{\prime}}\right]^{\circ} \mathrm{e}^{(t / 2) A} x\right\|_{Y_{\delta}^{p}} \\
& \quad \leq\left\|\left[(-A)^{\zeta}\right]^{\circ} \mathrm{e}^{(t / 2) A}\right\|_{\mathscr{L}\left(X ; Y_{\delta}^{p}\right)}\left\|\left[(-A)^{\zeta^{\prime}}\right]^{\circ} \mathrm{e}^{(t / 2) A} x\right\|_{X}\left\|\left[(-A)^{\zeta^{\prime}}\right]^{\circ} \mathrm{e}^{(t / 2) A}\right\|_{\mathscr{L}\left(Y_{\gamma}^{p} ; X\right)}\|x\|_{Y_{\gamma}^{p}} .
\end{aligned}
$$

Therefore, using (54) and (79) with the triplet $(\theta, \gamma, t)$ being equal to $(\zeta, \delta, t / 2)$ and $\left(\zeta^{\prime}, \gamma, t / 2\right)$, respectively, from (91) and $\mathfrak{R e} \theta=\mathfrak{R e} \zeta+\mathfrak{R e} \zeta^{\prime}$, we deduce that

$$
\begin{aligned}
& \left\|\left[(-A)^{\theta}\right]^{\circ} \mathrm{e}^{t A} x\right\|_{Y_{\delta}^{p}} \\
& \quad \leq c_{10} c_{22}\left(\frac{t}{2}\right)^{(\beta-\delta-\Re \mathrm{e} \zeta-1) / \alpha}\left(\frac{t}{2}\right)^{\left(\beta+\gamma-\Re \mathrm{e} \zeta^{\prime}-1\right) / \alpha}\|x\|_{Y_{\gamma}^{p}} \\
& \quad \leq c_{26} t^{(2 \beta+\gamma-\delta-\Re \mathrm{e} \theta-2) / \alpha}\|x\|_{Y_{\gamma}^{p}}, \quad t>0,
\end{aligned}
$$

where $c_{26}=2^{(2+\Re e \theta+\delta-\gamma-2 \beta) / \alpha} c_{10} c_{22}$. This completes the proof of the first estimate in (90), due to the arbitrariness of $x \in$ $Y_{\gamma}^{p}$. Let us now prove the second estimate in (90). Let $\theta \in \mathbf{C}$, $\mathfrak{R e} \theta \geq 1, \gamma, \delta \in(0,1), \gamma+\delta<1$, and $p \in[1, \infty]$ be fixed. Using $\gamma+\delta<1$, we fix $\gamma_{2} \in(\gamma /(1-\delta), 1) \varsubsetneqq(\gamma, 1)$, and we let $\gamma_{1}=\left(\gamma_{2} \delta\right) /\left(\gamma_{2}-\gamma\right)$. Clearly, since $\gamma_{2} \in(\gamma /(1-\delta), 1)$, we have $\gamma_{1} \in(\delta, 1)$. In addition, it holds:

$$
1-\delta>\frac{\gamma_{1}-\delta}{\gamma_{1}}=\left(\frac{\gamma_{2} \delta}{\gamma_{2}-\gamma}-\delta\right)\left(\frac{\gamma_{2}-\gamma}{\gamma_{2} \delta}\right)=\frac{\gamma}{\gamma_{2}}>\gamma .
$$

Due to (93), we now set $\gamma_{0}=\gamma / \gamma_{2}=\left(\gamma_{1}-\delta\right) / \gamma_{1} \in(\gamma, 1-\delta)$, so that $\gamma=\gamma_{0} \gamma_{2}$ and $\delta=\left(1-\gamma_{0}\right) \gamma_{1}$. From (24) with $p_{0}=p$ it thus follows that

$$
\begin{aligned}
& \left\|\left[(-A)^{\theta}\right]^{\circ} \mathrm{e}^{t A}\right\|_{\mathscr{L}\left(Y_{\gamma}^{p} ; Y_{\delta}^{p}\right)} \\
& \quad \leq\left\|\left[(-A)^{\theta}\right]^{\circ} \mathrm{e}^{t A}\right\|_{\mathscr{L}\left(X ; Y_{\gamma_{1}}^{p_{1}}\right)}^{1-\gamma_{0}}\left\|\left[(-A)^{\theta}\right]^{\circ} \mathrm{e}^{t A}\right\|_{\mathscr{L}\left(Y_{\gamma_{2}}^{p_{2}} ; X\right)}^{\gamma_{0}}, \quad t>0,
\end{aligned}
$$

where $p_{j} \in[1, \infty], j=1,2$. Applying (54) and (79) with the pair $(\gamma, p)$ being replaced with $\left(\gamma_{1}, p_{1}\right)$ and $\left(\gamma_{2}, p_{2}\right)$, respectively, from (94) we finally obtain

$$
\begin{aligned}
\left\|\left[(-A)^{\theta}\right]^{\circ} \mathrm{e}^{t A}\right\|_{\mathscr{L}\left(Y_{\gamma}^{p} ; Y_{\delta}^{p}\right)} \\
\quad \leq\left[c_{10} t^{\left(\beta-\gamma_{1}-\Re \mathrm{e} \theta-1\right) / \alpha}\right]^{1-\gamma_{0}}\left[c_{22} t^{\left(\beta+\gamma_{2}-\Re \mathrm{e} \theta-1\right) / \alpha}\right]^{\gamma_{0}} \\
\leq\left(c_{10}\right)^{1-\gamma_{0}}\left(c_{22}\right)^{\gamma_{0}} t^{\left[\beta+\gamma_{0} \gamma_{2}-\left(1-\gamma_{0}\right) \gamma_{1}-\Re \mathrm{e} \theta-1\right] / \alpha} \\
\quad=\left(c_{10}\right)^{\delta / \gamma_{1}}\left(c_{22}\right)^{\gamma / \gamma_{2}} t^{(\beta+\gamma-\delta-\Re \mathrm{e} \theta-1) / \alpha}, \quad t>0 .
\end{aligned}
$$

This completes the proof of the second estimate in (90) with $c_{27}=\left(c_{10}\right)^{\delta / \gamma_{1}}\left(c_{22}\right)^{\gamma / \gamma_{2}}$. 
Remark 17. We stress that if $\beta<1$ and $\gamma+\delta<1$, then the first estimate in (90) is rougher than the second one for small values of $t$, which justify our special attention to the case $\gamma+$ $\delta<1$. Indeed, if $\beta<1$, then for every $\mathfrak{R e} \theta \geq 1$ the following inequality holds:

$$
\begin{aligned}
\rho_{3}: & =\frac{(2 \beta+\gamma-\delta-\mathfrak{R e} \theta-2)}{\alpha} \\
& <\frac{(\beta+\gamma-\delta-\mathfrak{R e} \theta-1)}{\alpha}=: \rho_{4}<0,
\end{aligned}
$$

so that $t^{\rho_{4}} \leq t^{\rho_{3}}$ for $t \in(0,1]$. In other words, if $\beta$ and $\gamma+\delta$ are both less than one, then the second estimate in (90) establishes that the norm $\left\|\left[(-A)^{\theta}\right]^{\circ} \mathrm{e}^{t A}\right\|_{\mathscr{L}\left((X, \mathscr{D}(A))_{\gamma, p} ;(X, \mathscr{D}(A))_{\delta, p}\right)}$, $\mathfrak{R e} \theta \geq 1$, may blow up as $t$ goes to 0 , but with an order of singularity lower than that predicted by the first estimate. In this sense, though less general, the second estimate in (90) is better than the first one.

Remark 18. The reason why the second estimate in (90) yields a better exponent than the first one is the same mentioned in Remark 8. That is, while the first estimate is obtained in two steps: decomposing $\left[(-A)^{\theta}\right]^{\circ} \mathrm{e}^{t A}$ through (15) and then applying (54) and (79), the second estimate is essentially derived in a single step, using (24).

The following Remark 19 points out why, with the exception of the case when $\beta=1$ and $A$ is single-valued, to prove (90) we can not proceed as in [9, Proposition 2.2.9].

Remark 19. In the optimal case $\beta=1$, the exponents in both estimates (90) coincide equals to $\nu=\gamma-\delta-\mathfrak{R e} \theta$. Hence, in this special case, the assumption $\gamma+\delta<1$ does not give any enhancement. Also, if we further assume that $\theta \in \mathbf{N}$, then we restore the same estimates as in [9, Proposition 2.2.9(i)]. In this respect, our result extends [9] to the m. 1 . case, even though our proof really differs from that in [9]. For, there, the norms in the spaces $(X, \mathscr{D}(A))_{\sigma, p}$ are replaced with the norms in the spaces $\mathscr{D}_{A}(\sigma, p)$, with the latter being the spaces of all $x \in X$ such that $\|x\|_{\mathscr{D}_{A}(\sigma, p)}=\|x\|_{X}+[x]_{\mathscr{D}_{A}(\sigma, p)}<\infty$, where $[x]_{\mathscr{D}_{A}(\sigma, p)}=\left\|\xi^{(2-\beta-\sigma) / \alpha}\left[(-A)^{1}\right]^{\circ} \mathrm{e}^{\xi A}\right\|_{L_{p}^{*}(X)}$. It is well known that if $\beta=1$ and $A$ is single-valued, then $(X, \mathscr{D}(A))_{\sigma, p} \cong$ $\mathscr{D}_{A}(\sigma, p)$ (cf. [31, Theorem 3], [9, Proposition 2.2.2] and [27, Theorem 1.14.5]). On the contrary, if $(\alpha, \beta) \neq(1,1)$ and/or $A$ is really an $\mathrm{m}$. l. operator, such equivalence is no longer true and we have

$$
\begin{gathered}
X_{A}^{\sigma, p} \hookrightarrow(X, \mathscr{D}(A))_{\sigma, p} \hookrightarrow \mathscr{D}_{A}(\alpha \sigma, p), \quad p \in[1, \infty), \\
X_{A}^{\sigma, \infty} \hookrightarrow(X, \mathscr{D}(A))_{\sigma, \infty} \hookrightarrow \mathscr{D}_{A}(\sigma, \infty), \quad p=\infty .
\end{gathered}
$$

Differently from the spaces $X_{A}^{\sigma, p}$ and as a consequence of $A 0 \subseteq \bigcap_{t>0} \mathcal{N}\left(\left[(-A)^{1}\right]^{\circ} \mathrm{e}^{t A}\right)$, the spaces $\mathscr{D}_{A}(\sigma, p)$ contain $A 0$. It can thus be shown that if $\alpha+\beta>1$, then for every $\sigma \in(2-\alpha-\beta, 1)$ and $\varphi \in(0,(\alpha+\beta+\sigma-2) / \alpha)$ (here $(\alpha+\beta+\sigma-2) / \alpha<1$, since $\sigma<1 \leq 2-\beta)$ the following embeddings hold:

$$
\begin{aligned}
&\{0\} \cup\left[\mathscr{D}_{A}(\sigma, p) \backslash A 0\right] \hookrightarrow X_{A}^{\varphi, p} \hookrightarrow(X, \mathscr{D}(A))_{\varphi, p}, \\
& p \in[1, \infty), \\
&\{0\} \cup\left[\mathscr{D}_{A}(\sigma, \infty) \backslash A 0\right] \hookrightarrow X_{A}^{(\alpha+\beta+\sigma-2) / \alpha, \infty} \\
& \hookrightarrow(X, \mathscr{D}(A))_{(\alpha+\beta+\sigma-2) / \alpha, \infty},
\end{aligned}
$$

with $\{0\} \cup\left[\mathscr{D}_{A}(\sigma, p) \backslash A 0\right]$ being endowed with the norm of $\mathscr{D}_{A}(\sigma, p)$. Obviously, due to (29), it suffices to prove the embeddings on the right of (97) and on the left of (98). It is out of the aims of this paper to go into the details of these proofs, and for them we refer the readers to [24, Proposition 6.3]. Here we want only to make clear that, with the exception of the case when $\beta=1$ and $A$ is single-valued, embeddings (97) and (98) prevent us from carrying out the proof of estimates (90) simply by repeating the computations in [9]. Notice that, due to the property $\left[X_{A}^{\sigma, p} \cap A 0\right]=\{0\}$, from the second embeddings in (97) and (98) it follows that if $\alpha+\beta>1$ and $\sigma \in(2-\alpha-\beta, 1)$, then

$$
X_{A}^{\sigma, \infty} \hookrightarrow\{0\} \cup\left[\mathscr{D}_{A}(\sigma, \infty) \backslash A 0\right] \hookrightarrow X_{A}^{(\alpha+\beta+\sigma-2) / \alpha, \infty} .
$$

Since $(\alpha+\beta+\sigma-2) / \alpha \leq \sigma$ (indeed, $\alpha \leq 1 \leq(2-\beta-\sigma) /(1-\sigma)$ implies $\alpha+\beta+\sigma-2 \leq \alpha \sigma$ ), (99) agrees with (38) for $p=\infty$. In addition, if $2 \alpha+\beta>2$ and $\sigma \in((2-\alpha-\beta) / \alpha, 1)$, then the first embeddings in (97) and (98) yield for every $\varphi \in(0,(\alpha+$ $\beta+\alpha \sigma-2) / \alpha$ ) the following:

$$
X_{A}^{\sigma, p} \hookrightarrow\{0\} \cup\left[\mathscr{D}_{A}(\alpha \sigma, p) \backslash A 0\right] \hookrightarrow X_{A}^{\varphi, p}, \quad p \in[1, \infty) .
$$

Since $\varphi<(\alpha+\beta+\alpha \sigma-2) / \alpha \leq \sigma$, (100) agrees with (38) for $p \in[1, \infty)$. Furthermore, if $\beta=1$, then from (29), (30), and (99) it follows that $(X, \mathscr{D}(A))_{\sigma, \infty} \cong X_{A}^{\sigma, \infty} \cong\{0\} \cup\left[\mathscr{D}_{A}(\sigma, \infty) \backslash\right.$ $A 0], \sigma \in(0,1)$. This confirms that in the real $\mathrm{m}$. l. case the equivalence between $X_{A}^{\sigma, p},(X, \mathscr{D}(A))_{\sigma, p}$ and $\mathscr{D}_{A}(\sigma, p)$ does not hold even when $\beta=1$.

Using Propositions 9 and 12, we now obtain estimates for the operators $\left[(-A)^{\theta}\right]^{\circ} \mathrm{e}^{t A}, \mathfrak{R e} \theta \geq 1$, considered as operators from $X_{A}^{\gamma, p}$ to $X_{A}^{\delta, p}$. Clearly, since $\beta<1$ the spaces $X_{A}^{\sigma, p}$ may be not real interpolation spaces between $X$ and $\mathscr{D}(A)$, we can not proceed as in the proof of the second estimate in (90) and a weaker result has to be expected.

Proposition 20. Let $\mathfrak{R e} \theta \geq 1, \gamma, \delta \in(0,1)$, and $p \in[1, \infty]$. Then, there exist positive constants $c_{j}, j=28,29,30$, depending on $\alpha, \beta, \gamma, \delta, \theta$, and $p$ such that

$$
\begin{array}{r}
\left\|\left[(-A)^{\theta}\right]^{\circ} e^{t A}\right\|_{\mathscr{L}\left(X_{A}^{\gamma, \infty} ; X_{A}^{\delta, \infty}\right)} \leq c_{28} t^{(2 \beta+\gamma-\delta-\Re \mathrm{e} \theta-2) / \alpha}, \\
p=\infty, \quad t>0, \\
\left\|\left[(-A)^{\theta}\right]^{\circ} e^{t A}\right\|_{\mathscr{L}\left(X_{A}^{\gamma, p} ; X_{A}^{\beta \delta, p}\right)} \leq c_{29} t^{(2 \beta+\gamma-\delta-\Re \mathrm{e} \theta-2) / \alpha}, \\
p \in[1, \infty), \quad t>0 .
\end{array}
$$


Moreover, if $\gamma \in(0,1)$ and $\delta \in(1-\beta, 1)$ are such that $\gamma+\delta<1$, then

$$
\begin{array}{r}
\left\|\left[(-A)^{\theta}\right]^{\circ} e^{t A}\right\|_{\mathscr{L}\left(X_{A}^{\gamma, p} ; X_{A}^{\delta+\beta-1, p}\right)} \leq c_{30} t^{(\beta+\gamma-\delta-\Re \mathrm{e}-1) / \alpha}, \\
\\
p \in[1, \infty], \quad t>0 .
\end{array}
$$

Proof. Due to (61) and (79), in order to prove (101) and (102) it suffices to repeat the same computations as in (91) and (92), with the pair $\left((X, \mathscr{D}(A))_{\gamma, p},(X, \mathscr{D}(A))_{\delta, p}\right)$ being replaced with $\left(X_{A}^{\gamma, \infty}, X_{A}^{\delta, \infty}\right)$ or with $\left(X_{A}^{\gamma, p}, X_{A}^{\beta \delta, p}\right)$ provided that $p=\infty$ or $p \in[1, \infty)$. In this way we derive (101) and (102) with $c_{j+13}=2^{(2+R e \theta+\delta-\gamma-2 \beta) / \alpha} c_{j} c_{22}, j=15,16$. As far as (103) is concerned, we recall that if $X_{j}, j=1, \ldots, 4$, are four Banach spaces such that $X_{j} \hookrightarrow X_{j+2}, j=1,2$, and $L \in \mathscr{L}\left(X_{3} ; X_{2}\right)$, then $L \in \mathscr{L}\left(X_{1} ; X_{4}\right)$ with $\|L\|_{\mathscr{L}\left(X_{1} ; X_{4}\right)} \leq$ $C_{1} C_{2}\|L\|_{\mathscr{L}\left(X_{3} ; X_{2}\right)}, C_{1}$ and $C_{2}$ being the positive constants such that $\|x\|_{X_{j+2}} \leq C_{j}\|x\|_{X_{j}}, x \in X_{j}, j=1,2$. Applying this result to $L=\left[(-A)^{\theta}\right]^{\circ} \mathrm{e}^{t A}$ with $\left(X_{1}, X_{2}, X_{3}, X_{4}\right)=\left(X_{A}^{\gamma, p},(X\right.$, $\left.\mathscr{D}(A))_{\delta, p},(X, \mathscr{D}(A))_{\gamma, p}, X_{A}^{\delta+\beta-1, p}\right)$, from (29)-(32) and the second estimate in (90) we deduce (103) with $c_{30}=2 c_{2} c_{27}$. This completes the proof.

Remark 21. The assumption $\gamma+\delta<1$ with $\gamma \in(0,1)$ and $\delta \in(1-\beta, 1)$ implies that $\gamma \in(0,1-\delta) \varsubsetneqq(0, \beta)$. Therefore (cf. Remark 11), we conclude that for (103) to hold we have to consider $\left[(-A)^{\theta}\right]^{\circ} \mathrm{e}^{t A}, \mathfrak{R e} \theta \geq 1$, as an operator between the intermediate spaces $X_{A}^{\gamma, p}$ and $X_{A}^{\varepsilon, p}$, where $\gamma, \varepsilon \in(0, \beta)$, $\varepsilon=\delta+\beta-1, \delta \in(1-\beta, 1), \gamma+\delta<1$.

\section{Hölder Regularity of Some Operator Functions}

Here, we study the Hölder regularity of those operator functions that we will need in Section 5. From now on, with $\left(Z,\|\cdot\|_{Z}\right)$ being a complex Banach space, $C([a, b] ; Z)=$ $C^{0}([a, b] ; Z)$ and $C^{\delta}([a, b] ; Z), \delta \in(0,1), a<b$, denote, respectively, the spaces of all continuous and $\delta$-Hölder continuous functions from $[a, b]$ into $Z$ endowed with the norms $\|g\|_{0, a, b ; Z}=\sup _{t \in[a, b]}\|g(t)\|_{Z}$ and $\|g\|_{\delta, a, b ; Z}=\|g\|_{0, a, b ; Z}+$ $|g|_{\delta, a, b ; Z}$, where $|g|_{\delta, a, b ; Z}$ is the seminorm $\sup _{a \leq t_{1}<t_{2} \leq b}\left(t_{2}-\right.$ $\left.t_{1}\right)^{-\delta}\left\|g\left(t_{2}\right)-g\left(t_{1}\right)\right\|_{Z}$. We endow the subspace $C_{0}^{\delta}([a, b] ; Z)=$ $\left\{g \in C^{\delta}([a, b] ; Z): g(a)=0\right\}, \delta \in[0,1)$ with the norm $\|\cdot\|_{\delta, a, b ; Z}$. Further, for $k \in \mathbf{N}$ and $\delta \in(0,1)$ we set $C^{k}([a, b]$; $Z)=\left\{g \in C([a, b] ; Z): D_{t}^{k} g \in C([a, b] ; Z)\right\},\|g\|_{k, a, b ; Z}=$ $\sum_{0}^{k}\left\|D_{t}^{j} g\right\|_{0, a, b ; Z}\left(D_{t}^{0}=I\right)$, and $C^{k+\delta}([a, b] ; Z)=\left\{g \in C^{k}([a, b]\right.$; $\left.Z): D_{t}^{k} g \in C^{\delta}([a, b] ; Z)\right\},\|g\|_{k+\delta, a, b ; Z}=\|g\|_{k, a, b ; Z}+\mid D_{t}^{k}$ $\left.g\right|_{\delta, a, b ; Z}$. Recall that if $0 \leq \delta_{2} \leq \delta_{1} \leq 1$, then $C^{\delta_{1}}([a, b] ; Z) \hookrightarrow$ $C^{\delta_{2}}([a, b] ; Z)$ and $\|g\|_{\delta_{2}, a, b ; Z} \leq \max \left\{1,(b-a)^{\delta_{1}-\delta_{2}}\right\}\|g\|_{\delta_{1}, a, b ; Z}$, $g \in C^{\delta_{1}}([a, b] ; X)$. Finally, given three complex Banach spaces $\left(X_{k},\|\cdot\|_{X_{k}}\right), k=1,2,3$, and a bilinear bounded operator $\mathscr{P}$ from $X_{1} \times X_{2}$ to $X_{3}$ with norm $C_{0}$, that is, $\mathscr{P} \in \mathscr{B}\left(X_{1} \times X_{2} ; X_{3}\right)$ and $\|\mathscr{P}\|_{\mathscr{B}\left(X_{1} \times X_{2} ; X_{3}\right)}=\sup _{\left\|x_{k}\right\|_{X_{k}}=1, k=1,2}\left\|\mathscr{P}\left(x_{1}, x_{2}\right)\right\|_{X_{3}}=C_{0}$, we denote by $\mathscr{K}$ the convolution operator

$$
\begin{array}{r}
\mathscr{K}\left(v_{1}, v_{2}\right)(t)=\int_{0}^{t} \mathscr{P}\left(v_{1}(t-r), v_{2}(r)\right) \mathrm{d} r, \\
t \in[0, b], \quad b>0,
\end{array}
$$

where $v_{k}:[0, b] \rightarrow X_{k}, k=1,2$. Of course, if $\left(X_{1}, X_{2}\right)=$ $\left(\mathrm{C}, X_{3}\right)$ and if $\mathscr{P}$ is the scalar multiplication in $X_{3}$, that is, $\mathscr{P}(z, x)=z x, z \in \mathbf{C}, x \in X_{3}$, then $C_{0}=1$ and $\mathscr{K}$ reduces to the usual convolution operator $\mathscr{K}\left(v_{1}, v_{2}\right)(t)=\int_{0}^{t} v_{1}(t-$ $r) v_{2}(r) \mathrm{d} r$. As usual, for every $q \in[1, \infty]$, we will denote by $q^{\prime}$ the conjugate exponent of $q$.

Now let $X_{3}=X$ and introduce the following linear operators $Q_{j}, j=1, \ldots, 6$, where $g_{j} \in C^{\delta_{j}}([0, T] ; X), j=1,2,5$, $g_{l_{k}} \in C^{\delta_{l_{k}}}\left([0, T], X_{k}\right), l=3,6, k=1,2, g_{4} \in C^{\delta_{4}}([0, T] ; \mathbf{C})$, $y \in Y_{\gamma}^{p}, Y_{\gamma}^{p} \in\left\{(X, \mathscr{D}(A))_{\gamma, p}, X_{A}^{\gamma, p}\right\}, p \in[1, \infty]$, and $t \in[0, T]$, $T>0$ as follows:

$$
\begin{gathered}
{\left[Q_{1} g_{1}\right](t):=\int_{0}^{t} \mathrm{e}^{(t-s) A} g_{1}(s) \mathrm{d} s} \\
{\left[Q_{2} g_{2}\right](t):=\int_{0}^{t}\left[(-A)^{1}\right]^{\circ} \mathrm{e}^{(t-s) A}\left[g_{2}(s)-g_{2}(t)\right] \mathrm{d} s,} \\
{\left[Q_{3}\left(g_{3_{1}}, g_{3_{2}}\right)\right](t):=\left[Q_{2} \mathscr{K}\left(g_{3_{1}}, g_{3_{2}}\right)\right](t),} \\
{\left[Q_{4}\left(g_{4}, y\right)\right](t):=\left[Q_{2}\left(g_{4} y\right)\right](t),} \\
{\left[Q_{5} g_{5}\right](t):=\left[\mathrm{e}^{t A}-I\right] g_{5}(t),} \\
{\left[Q_{6}\left(g_{6_{1}}, g_{6_{2}}\right)\right](t):=\left[Q_{5} \mathscr{K}\left(g_{6_{1}}, g_{6_{2}}\right)\right](t),}
\end{gathered}
$$

with $g_{4} y$ being the function from $[0, T]$ to $Y_{\gamma}^{p}$ defined by $\left(g_{4} y\right)(t)=g_{4}(t) y$. We will find conditions on $\delta_{j}, \delta_{l_{k}}, \delta_{4}, \gamma \in$ $(0,1), j=1,2,5, l=3,6, k=1,2$, in order that $Q_{j} g_{j} \in C^{\tau_{j}}$ $([0, T] ; X), Q_{l}\left(g_{l_{1}}, g_{l_{2}}\right) \in C^{\tau_{l}}([0, T] ; X)$ and $Q_{4}\left(g_{4}, y\right) \in C^{\tau_{4}}$ $([0, T] ; X)$ for opportunely chosen $\tau_{j}, \tau_{l}, \tau_{4} \in(0,1)$. We emphasize of the presence of the increment $g_{2}(s)-g_{2}(t)$ inside the integral defining $Q_{2} g_{2}$. As we will see, and differently from $Q_{1}$, it is just this presence which makes $Q_{2} g_{2}$ well-defined for smooth enough functions $g_{2}$. This is the reason why the operator $Q_{2}$ as it was defined in [20, formula (4.12)] can make no sense and has to be replaced with that defined by the present (106) (cf. the appendix below). We begin our analysis on the $Q_{j}$ 's with the following result proven in [20, Lemma 4.1]. Since we will need it later, here, removing some misprints in [20], we report its short proof for the reader's convenience.

Lemma 22. Let $\alpha+\beta>1$ in (H1). Then, for every $\delta_{1} \in(0$, $(\alpha+\beta-1) / \alpha)$, the operator $Q_{1}$ defined by (105) maps $C^{\delta_{1}}$ $([0, T] ; X)$ into $C_{0}^{\delta_{1}}([0, T] ; X)$, and for every $t \in[0, T]$ satisfies the following estimate, where $p \in\left(\alpha /\left(\alpha+\beta-1-\alpha \delta_{1}\right), \infty\right)$ as follows:

$$
\left\|Q_{1} g_{1}\right\|_{\delta_{1}, 0, t ; X} \leq C_{1}(t)\left(\int_{0}^{t}\left\|g_{1}\right\|_{\delta_{1}, 0, s ; X}^{p} \mathrm{~d} s\right)^{1 / p} .
$$

Here $C_{1}(t)$ is a nondecreasing function of $t$ depending also on $\alpha, \beta, \delta_{1}$, and $p^{\prime}$. 
Proof. Let $g_{1} \in C^{\delta_{1}}([0, T] ; X), \delta_{1} \in(0,(\alpha+\beta-1) / \alpha)$, and $t \in$ $[0, T]$. From (14) and the Hölder inequality with $p \in(\alpha /(\alpha+$ $\left.\left.\beta-1-\alpha \delta_{1}\right), \infty\right) \varsubsetneqq(1, \infty)$, for any $\tau \in[0, t]$, we deduce that

$$
\begin{aligned}
& \left\|\left[Q_{1} g_{1}\right](\tau)\right\|_{X} \\
& \quad \leq \widetilde{c}_{\alpha, \beta, 0} \int_{0}^{\tau}(\tau-s)^{(\beta-1) / \alpha}\left\|g_{1}\right\|_{0,0, s ; X} \mathrm{~d} s \\
& \quad \leq c_{31} \tau^{\left[\alpha-(1-\beta) p^{\prime}\right] /\left(\alpha p^{\prime}\right)}\left(\int_{0}^{\tau}\left\|g_{1}\right\|_{\delta_{1}, 0, s ; X}^{p} \mathrm{~d} s\right)^{1 / p} \\
& \quad \leq c_{31} \tau^{\left[\alpha-\left(1+\alpha \delta_{1}-\beta\right) p^{\prime}\right] /\left(\alpha p^{\prime}\right)} \tau^{\delta_{1}}\left(\int_{0}^{\tau}\left\|g_{1}\right\|_{\delta_{1}, 0, s ; X}^{p} \mathrm{~d} s\right)^{1 / p},
\end{aligned}
$$

where $c_{31}=\widetilde{c}_{\alpha, \beta, 0} \alpha^{1 / p^{\prime}}\left[\alpha-(1-\beta) p^{\prime}\right]^{-1 / p^{\prime}}$. Here $\alpha-\left(1+\alpha \delta_{1}-\beta\right)$ $p^{\prime}>0$, since $p^{\prime} \in\left(1, \alpha /\left(1+\alpha \delta_{1}-\beta\right)\right)$. For $1-1 / p>1-(\alpha+$ $\left.\beta-1-\alpha \delta_{1}\right) / \alpha=\left(1+\alpha \delta_{1}-\beta\right) / \alpha$. passing to the supremum with respect to $\tau \in[0, t]$ in (112) we thus find

$\left\|Q_{1} g_{1}\right\|_{0,0, t ; X}$

$$
\leq c_{31} t^{\left[\alpha-\left(1+\alpha \delta_{1}-\beta\right) p^{\prime}\right] /\left(\alpha p^{\prime}\right)} t^{\delta_{1}}\left(\int_{0}^{t}\left\|g_{1}\right\|_{\delta_{1}, 0, s ; X}^{p} \mathrm{~d} s\right)^{1 / p} .
$$

Now let (since $\left[Q_{1} g_{1}\right](0)=0$, the case $t_{1}=0$ follows from (112) with $\left.\tau=t_{2}\right) 0<t_{1}<t_{2} \leq t$. The change of variable $t-s=r$ in (105) leads us to $\left[Q_{1} g_{1}\right]\left(t_{2}\right)-\left[Q_{1} g_{1}\right]\left(t_{1}\right)=\sum_{k=1}^{2}$ $I_{k ; t_{1}, t_{2}, g_{1}}$, where $I_{1 ; t_{1}, t_{2}, g_{1}}:=\int_{t_{1}}^{t_{2}} e^{r A} g_{1}\left(t_{2}-r\right) \mathrm{d} r$ and $I_{2 ; t_{1}, t_{2}, g_{1}}:=$ $\int_{0}^{t_{1}} \mathrm{e}^{r A}\left[g_{1}\left(t_{2}-r\right)-g_{1}\left(t_{1}-r\right)\right] \mathrm{d} r$. Reasoning as in (112) and using the inequality $t_{2}^{\mu}-t_{1}^{\mu} \leq\left(t_{2}-t_{1}\right)^{\mu}, \mu \in(0,1]$, we get

$$
\begin{aligned}
& \left\|I_{1 ; t_{1}, t_{2}, g_{1}}\right\|_{X} \\
& \leq c_{31}\left(t_{2}-t_{1}\right)^{\left[\alpha-(1-\beta) p^{\prime}\right] /\left(\alpha p^{\prime}\right)}\left(\int_{t_{1}}^{t_{2}}\left\|g_{1}\right\|_{\delta_{1}, 0, t_{2}-r ; X}^{p} \mathrm{~d} r\right)^{1 / p} \\
& \leq c_{31} t_{2}^{\left[\alpha-\left(1+\alpha \delta_{1}-\beta\right) p^{\prime}\right] /\left(\alpha p^{\prime}\right)}\left(t_{2}-t_{1}\right)^{\delta_{1}}\left(\int_{0}^{t}\left\|g_{1}\right\|_{\delta_{1}, 0, t-r ; X}^{p} \mathrm{~d} r\right)^{1 / p} .
\end{aligned}
$$

Similarly, but taking advantage from $g_{1} \in C^{\delta_{1}}([0, T] ; X)$, we obtain

$$
\begin{aligned}
& \left\|I_{2 ; t_{1}, t_{2}, g_{1}}\right\|_{X} \\
& \leq \widetilde{c}_{\alpha, \beta, 0}\left(t_{2}-t_{1}\right)^{\delta_{1}} \int_{0}^{t_{1}} r^{(\beta-1) / \alpha}\left|g_{1}\right|_{\delta_{1}, 0, t_{2}-r ; X} \mathrm{~d} r \\
& \leq c_{31} t_{1}^{\left[\alpha-\left(1+\alpha \delta_{1}-\beta\right) p^{\prime}\right] /\left(\alpha p^{\prime}\right)} t_{1}^{\delta_{1}}\left(t_{2}-t_{1}\right)^{\delta_{1}}\left(\int_{0}^{t}\left\|g_{1}\right\|_{\delta_{1}, 0, t-r ; X}^{p} \mathrm{~d} r\right)^{1 / p} .
\end{aligned}
$$

Thus, letting $\widetilde{\mathcal{c}}_{1}(t)=c_{31} t^{\left[\alpha-\left(1+\alpha \delta_{1}-\beta\right) p^{\prime}\right] /\left(\alpha p^{\prime}\right)}$ from (114) and (115) it follows that

$$
\begin{aligned}
& \left\|\left[Q_{1} g_{1}\right]\left(t_{2}\right)-\left[Q_{1} g_{1}\right]\left(t_{1}\right)\right\|_{X} \\
& \quad \leq \widetilde{c}_{1}(t)\left(t^{\delta_{1}}+1\right)\left(t_{2}-t_{1}\right)^{\delta_{1}}\left(\int_{0}^{t}\left\|g_{1}\right\|_{\delta_{1}, 0, t-r ; X}^{p} \mathrm{~d} r\right)^{1 / p} .
\end{aligned}
$$

Finally, summing up (113) and (116) and using $\int_{0}^{t}\left\|g_{1}\right\|_{\delta_{1}, 0, t-r ; X}^{p}$ $\mathrm{d} r=\int_{0}^{t}\left\|g_{1}\right\|_{\delta_{1}, 0, s ; X}^{p} \mathrm{~d} s$, we derive (111) with $C_{1}(t)=\widetilde{c}_{1}(t)\left(2 t^{\delta_{1}}+\right.$ 1). This completes the proof.

Remark 23. We stress that if we renounce to its Hölder regularity, then for $Q_{1} g_{1}$ to be well-defined it suffices that $\alpha$ and $\beta$ are as in Lemma 22 and that $g_{1}$ is merely in $C([0, T] ; X)$. In fact (see the last part of the proof of Corollary 14, replacing there $x$ with $\left.g_{1}(s)\right)$, $\left\|\left[Q_{1} g_{1}\right](t)\right\|_{X} \leq \alpha(\alpha+\beta-$ $1)^{-1} \widetilde{c}_{\alpha, \beta, 0}\left\|g_{1}\right\|_{0,0, t ; X} t^{(\alpha+\beta-1) / \alpha}, t \in[0, T]$.

Lemma 24. Let $3 \alpha+\beta>3$ in (H1). Then, for every $\delta_{2} \in$ $((3-2 \alpha-\beta) / \alpha, 1)$, the operator $Q_{2}$ defined by (106) maps $C^{\delta_{2}}([0, T] ; X)$ into $C_{0}^{\nu_{2}}([0, T] ; X), \nu_{2}=\left(\alpha \delta_{2}+2 \alpha+\beta-3\right) / \alpha \in$ $\left(0, \delta_{2}\right]$, and for every $t \in[0, T]$ it satisfies the following estimate:

$$
\left\|Q_{2} g_{2}\right\|_{\gamma_{2}, 0, t ; X} \leq C_{2}(t)\left|g_{2}\right|_{\delta_{2}, 0, t ; X^{*}}
$$

Here $C_{2}(t)$ is a nondecreasing function of $t$ depending also on $\alpha, \beta$, and $\delta_{2}$.

Proof. Denote by $\bar{\alpha}$ the number $(1-\alpha) / \alpha$. In particular, since $3 \alpha+\beta>3$ implies $\alpha \in(2 / 3,1]$, we have $\bar{\alpha} \in[0,1 / 2)$. Let $t \in[0, T], g_{2} \in C^{\delta_{2}}([0, T] ; X), \delta_{2} \in((3-2 \alpha-\beta) / \alpha, 1)$, and $v_{2}=\left(\alpha \delta_{2}+2 \alpha+\beta-3\right) / \alpha \in\left(0, \delta_{2}\right]$. We notice that $\left(\alpha \delta_{2}+\beta-\right.$ 2) $/ \alpha=\nu_{2}+\bar{\alpha}-1$ and $\left(\alpha \delta_{2}+\beta-3\right) / \alpha=\nu_{2}-2$. Then, using (14) with $\theta=1$, for every $\tau \in[0, t]$ we obtain

$$
\begin{aligned}
& \left\|\left[Q_{2} g_{2}\right](\tau)\right\|_{X} \\
& \quad \leq \widetilde{c}_{\alpha, \beta, 1}\left|g_{2}\right|_{\delta_{2}, 0, \tau ; X} \int_{0}^{\tau}(\tau-s)^{\left(\alpha \delta_{2}+\beta-2\right) / \alpha} \mathrm{d} s \\
& \quad=c_{32}\left|g_{2}\right|_{\delta_{2}, 0, \tau ; X} \tau^{\nu_{2}+\bar{\alpha}},
\end{aligned}
$$

where $c_{32}=\widetilde{c}_{\alpha, \beta, 1}\left(\nu_{2}+\bar{\alpha}\right)^{-1}$. Hence

$$
\left\|Q_{2} g_{2}\right\|_{0,0, t ; X} \leq c_{32}\left|g_{2}\right|_{\delta_{2}, 0, t ; X} t^{\nu_{2}+\bar{\alpha}}
$$

Now let (since $\left[Q_{2} g_{2}\right](0)=0$, the case $t_{1}=0$ follows from (118) with $\left.\tau=t_{2}\right) 0<t_{1}<t_{2} \leq t$. We have 
$\left[Q_{2} g_{2}\right]\left(t_{2}\right)-\left[Q_{2} g_{2}\right]\left(t_{1}\right)=\sum_{k=1}^{3} J_{k ; t_{1}, t_{2}, g_{2}}$, where for a function $g:[0, T] \rightarrow X$ we set

$$
\begin{aligned}
& J_{1 ; t_{1}, t_{2}, g} \\
&:=\int_{0}^{t_{1}}\left\{\left[(-A)^{1}\right]^{\circ} \mathrm{e}^{\left(t_{2}-s\right) A}-\left[(-A)^{1}\right]^{\circ} \mathrm{e}^{\left(t_{1}-s\right) A}\right\} \\
& \times\left[g(s)-g\left(t_{1}\right)\right] \mathrm{d} s \\
& J_{2 ; t_{1}, t_{2}, g}:= \int_{0}^{t_{1}}\left[(-A)^{1}\right]^{\circ} \mathrm{e}^{\left(t_{2}-s\right) A}\left[g\left(t_{1}\right)-g\left(t_{2}\right)\right] \mathrm{d} s, \\
& J_{3 ; t_{1}, t_{2}, g}:= \int_{t_{1}}^{t_{2}}\left[(-A)^{1}\right]^{\circ} \mathrm{e}^{\left(t_{2}-s\right) A}\left[g(s)-g\left(t_{2}\right)\right] \mathrm{d} s .
\end{aligned}
$$

First, using (13) with $(s, t, \theta)=\left(t_{1}-s, t_{2}-s, 1\right), s \in\left(0, t_{1}\right)$, and (14) with $\theta=2$, and letting $\left(c_{33}, c_{34}\right)=\left(\widetilde{c}_{\alpha, \beta, 2}\left(1-v_{2}\right)^{-1}, c_{33} \nu_{2}^{-1}\right)$, we get

$$
\begin{aligned}
& \|\left. J_{1 ; t_{1}, t_{2}, g_{2}}\right|_{X} \\
& \quad \leq \widetilde{c}_{\alpha, \beta, 2}\left|g_{2}\right|_{\delta_{2}, 0, t_{1} ; X} \int_{0}^{t_{1}}\left[\int_{t_{1}-s}^{t_{2}-s} \xi^{(\beta-3) / \alpha} \mathrm{d} \xi\right]\left(t_{1}-s\right)^{\delta_{2}} \mathrm{~d} s \\
& \quad \leq \widetilde{c}_{\alpha, \beta, 2}\left|g_{2}\right|_{\delta_{2}, 0, t_{1} ; X} \int_{0}^{t_{1}}\left[\int_{t_{1}-s}^{t_{2}-s} \xi^{\left(\alpha \delta_{2}+\beta-3\right) / \alpha} \mathrm{d} \xi\right] \mathrm{d} s \\
& \quad=c_{33}\left|g_{2}\right|_{\delta_{2}, 0, t_{1} ; X} \int_{0}^{t_{1}}\left[\left(t_{1}-s\right)^{\nu_{2}-1}-\left(t_{2}-s\right)^{\nu_{2}-1}\right] \mathrm{d} s \\
& \quad=c_{34}\left|g_{2}\right|_{\delta_{2}, 0, t_{1} ; X}\left[t_{1}^{\nu_{2}}+\left(t_{2}-t_{1}\right)^{\nu_{2}}-t_{2}^{\nu_{2}}\right] \\
& \leq c_{34}\left|g_{2}\right|_{\delta_{2}, 0, t_{2} ; X}\left(t_{2}-t_{1}\right)^{\nu_{2}} .
\end{aligned}
$$

Let us turn to $J_{2 ; t_{1}, t_{2}, g_{2}}$. We first observe that the integral $\int_{0}^{t_{1}}\left[(-A)^{1}\right]^{\circ} \mathrm{e}^{\left(t_{2}-s\right) A} \mathrm{~d} s$ is convergent. For, $\| \int_{0}^{t_{1}}\left[(-A)^{1}\right]^{0} \mathrm{e}^{\left(t_{2}-s\right) A}$ $\mathrm{d} s \|_{X} \leq \widetilde{c}_{\alpha, \beta, 1} \int_{0}^{t_{1}}\left(t_{2}-s\right)^{(\beta-2) / \alpha} \mathrm{d} s \leq C_{\alpha, \beta, t_{1}, t_{2}}$, where $C_{\alpha, \beta, t_{1}, t_{2}}$ is equal to $\widetilde{c}_{\alpha, \beta, 1} \ln \left[t_{2}\left(t_{2}-t_{1}\right)^{-1}\right]$ if $\beta=1$ and to $\alpha(2-\alpha-\beta)^{-1}$ $\widetilde{c}_{\alpha, \beta, 1}\left[\left(t_{2}-t_{1}\right)^{(\alpha+\beta-2) / \alpha}-t_{2}^{(\alpha+\beta-2) / \alpha}\right]$ if $\beta \in(0,1)$. Thus, we may rewrite it as $-\int_{t_{2}}^{t_{2}-t_{1}}\left[(-A)^{1}\right]^{0} \mathrm{e}^{r A} \mathrm{~d} r=\int_{t_{2}}^{t_{2}-t_{1}} D_{r} \mathrm{e}^{r A} \mathrm{~d} r=$ $\mathrm{e}^{\left(t_{2}-t_{1}\right) A}-\mathrm{e}^{t_{2} A}$. Consequently,

$$
\begin{aligned}
\| & J_{2 ; t_{1}, t_{2}, g_{2}} \|_{X} \\
\leq & \widetilde{c}_{\alpha, \beta, 0}\left[\left(t_{2}-t_{1}\right)^{(\beta-1) / \alpha}+t_{2}^{(\beta-1) / \alpha}\right]\left|g_{2}\right|_{\delta_{2}, 0, t_{2} ; X}\left(t_{2}-t_{1}\right)^{\delta_{2}} \\
\leq & \widetilde{c}_{\alpha, \beta, 0}\left\{1+\left[t_{2}\left(t_{2}-t_{1}\right)^{-1}\right]^{(\beta-1) / \alpha}\right\} \\
& \times\left|g_{2}\right|_{\delta_{2}, 0, t_{2} ; X}\left(t_{2}-t_{1}\right)^{\left(\alpha \delta_{2}+\beta-1\right) / \alpha} \\
\leq & 2 \widetilde{c}_{\alpha, \beta, 0}\left|g_{2}\right|_{\delta_{2}, 0, t_{2} ; X}\left(t_{2}-t_{1}\right)^{\nu_{2}+2 \bar{\alpha}},
\end{aligned}
$$

where we have used $\left[t_{2}\left(t_{2}-t_{1}\right)^{-1}\right]^{(\beta-1) / \alpha} \leq 1$ and $\left(\alpha \delta_{2}+\right.$ $\beta-1) / \alpha=\nu_{2}+2 \bar{\alpha}$. As far as $J_{3 ; t_{1}, t_{2}, g_{2}}$ is concerned, instead, reasoning as in the derivation of (118) we find

$$
\begin{aligned}
\left\|J_{3 ; t_{1}, t_{2}, g_{2}}\right\|_{X} & \leq \widetilde{c}_{\alpha, \beta, 1}\left|g_{2}\right|_{\delta_{2}, 0, t_{2} ; X} \int_{t_{1}}^{t_{2}}\left(t_{2}-s\right)^{\nu_{2}+\bar{\alpha}-1} \mathrm{~d} s \\
& =c_{32}\left|g_{2}\right|_{\delta_{2}, 0, t_{2} ; X}\left(t_{2}-t_{1}\right)^{\nu_{2}+\bar{\alpha}} .
\end{aligned}
$$

Then, summing up (121)-(123) and letting $\widetilde{c}_{2}(t)=c_{34}+$ $2 \widetilde{c}_{\alpha, \beta, 0} t^{2 \bar{\alpha}}+c_{32} t^{\bar{\alpha}}$, we obtain

$$
\begin{aligned}
\left\|\left[Q_{2} g_{2}\right]\left(t_{2}\right)-\left[Q_{2} g_{2}\right]\left(t_{1}\right)\right\|_{X} & \leq \sum_{k=1}^{3}\left\|J_{k ; t_{1}, t_{2}, g_{2}}\right\|_{X} \\
& \leq \widetilde{c}_{2}(t)\left|g_{2}\right|_{\delta_{2}, 0, t ; X}\left(t_{2}-t_{1}\right)^{v_{2}} .
\end{aligned}
$$

Finally, (119) and (124) yield (117) with $C_{2}(t)=c_{32} t^{\nu_{2}+\bar{\alpha}}+\widetilde{\mathcal{c}}_{2}(t)$.

Remark 25. In particular, Lemma 24 establishes that, with the exception of the case $\beta=1$ in which $\nu_{2}=\delta_{2}, Q_{2}$ produces a loss of regularity equal to $\delta_{2}-v_{2}=(3-2 \alpha-\beta) / \alpha$.

As Corollary 14, the next result will be needed to prove the equivalence between problem (170) and the fixed-point equation (179). From now on, if $A^{-1} \in \mathscr{L}(X)$ and $g \in$ $C^{\delta}([0, T] ; X), \delta \in[0,1)$, with $A^{-1} g$ we will always mean the function in $C^{\delta}([0, T] ; \mathscr{D}(A))$ defined by $\left(A^{-1} g\right)(t)=$ $A^{-1}(g(t))$. Notice that $\left\|A^{-1} g\right\|_{\delta, 0, t ; \mathscr{D}(A)} \leq\|g\|_{\delta, 0, t ; X}, t \in[0, T]$.

\section{Corollary 26.}

(i) Let $2 \alpha+\beta>2$ in (H1). Then, for every $g \in C^{\delta}$ $([0, T] ; X), \delta \in((2-\alpha-\beta) / \alpha, 1)$,

$$
A^{-1}\left[Q_{2} g\right](t)=-\int_{0}^{t} e^{(t-s) A}[g(s)-g(t)] \mathrm{d} s, \quad \mathrm{t} \in[0, \mathrm{~T}] .
$$

(ii) Let $\alpha+\beta>1$ in (H1). Then, for every $g \in C([0, T] ; X)$

$$
\left[Q_{2}\left(A^{-1} g\right)\right](t)=-\int_{0}^{t} e^{(t-s) A}[g(s)-g(t)] \mathrm{d} s, \quad \mathrm{t} \in[0, \mathrm{~T}] .
$$

Proof. Of course, it suffices to assume that $t \in(0, T]$. Let us first prove (i). So, let $2 \alpha+\beta>2, g \in C^{\delta}([0, T] ; X), \delta \in((2-$ $\alpha-\beta) / \alpha, 1)$, and $t \in(0, T]$, and we observe that both sides of $(125)$ are well defined. Indeed, replacing the pair $\left(g_{2}, \delta_{2}\right)$ with $(g, \delta)$, from (118) we get

$$
\begin{aligned}
& \left\|\left[Q_{2} g\right](t)\right\|_{X} \\
& \quad \leq \widetilde{c}_{\alpha, \beta, 1} \alpha(\alpha \delta+\alpha+\beta-2)^{-1}|g|_{\delta, 0, t ; X} t^{(\alpha \delta+\alpha+\beta-2) / \alpha} .
\end{aligned}
$$


On the other side, $I_{t, g}=\int_{0}^{t} \mathrm{e}^{(t-s) A}[g(s)-g(t)] \mathrm{d} s$ satisfies

$$
\begin{aligned}
\left\|I_{t, g}\right\|_{X} & \leq \widetilde{c}_{\alpha, \beta, 0}|g|_{\delta, 0, t ; X} \int_{0}^{t}(t-s)^{(\alpha \delta+\beta-1) / \alpha} \mathrm{d} s \\
& \leq c_{35}|g|_{\delta, 0, t ; X} t^{(\alpha+\alpha \delta+\beta-1) / \alpha},
\end{aligned}
$$

where $c_{35}=\alpha(\alpha \delta+\alpha+\beta-1)^{-1} \widetilde{c}_{\alpha, \beta, 0}$. Then, commuting $A^{-1} \in \mathscr{L}(X)$ with the integral signs, using (80) with $\theta=1$, and taking into account (7), we find

$$
\begin{aligned}
& A^{-1}\left[Q_{2} g_{2}\right](t) \\
& =A^{-1} \int_{0}^{t}\left[-\frac{1}{2 \pi i} \int_{\Gamma} \mathrm{e}^{(t-s) \lambda} A^{\circ}(\lambda I-A)^{-1} \mathrm{~d} \lambda\right]\left[g_{2}(s)-g_{2}(t)\right] \mathrm{d} s \\
& =-\int_{0}^{t}\left[\frac{1}{2 \pi i} \int_{\Gamma} \mathrm{e}^{(t-s) \lambda} A^{-1} A^{\circ}(\lambda I-A)^{-1} \mathrm{~d} \lambda\right]\left[g_{2}(s)-g_{2}(t)\right] \mathrm{d} s \\
& =-\int_{0}^{t}\left[\frac{1}{2 \pi i} \int_{\Gamma} \mathrm{e}^{(t-s) \lambda}(\lambda I-A)^{-1} \mathrm{~d} \lambda\right]\left[g_{2}(s)-g_{2}(t)\right] \mathrm{d} s .
\end{aligned}
$$

Since $(2 \pi i)^{-1} \int_{\Gamma} \mathrm{e}^{(t-s) \lambda}(\lambda I-A)^{-1} \mathrm{~d} \lambda=\mathrm{e}^{(t-s) A}$, the proof of (125) is complete. We now prove (ii). Let $\alpha+\beta>1, g \in$ $C([0, T] ; X)$ and $t \in(0, T]$. Then, for every $\gamma \in(2-\alpha-\beta, 1)$, the same reasonings made to derive (88), except for replacing $x$ with $g(s)-g(t)$, yield

$$
\begin{aligned}
& \left\|\left[Q_{2}\left(A^{-1} g\right)\right](t)\right\|_{X} \\
& \quad \leq 2 c_{22} c_{\alpha, \beta, \gamma} c_{0}\left\|A^{-1}\right\|_{\mathscr{L}(X)}^{1-\gamma}\|g\|_{0,0, t ; X} t^{(\alpha+\beta+\gamma-2) / \alpha} .
\end{aligned}
$$

Hence, $\left[Q_{2}\left(A^{-1} g\right)\right](t)$ being meaningful, we obtain (126) simply applying to it formula (89) with $\zeta=1$ and then using $\left[(-A)^{0}\right]^{\circ} \mathrm{e}^{(t-s) A}=\mathrm{e}^{(t-s) A}, s \in(0, t)$. In particular, a better estimate than (130) holds. For, $\left[Q_{2}\left(A^{-1} g\right)\right](t)=-\int_{0}^{t} \mathrm{e}^{(t-s) A}$ $[g(s)-g(t)] \mathrm{d} s$ satisfies

$$
\begin{aligned}
\left\|\left[Q_{2}\left(A^{-1} g\right)\right](t)\right\|_{X} & \leq 2 \widetilde{c}_{\alpha, \beta, 0}\|g\|_{0,0, t ; X} \int_{0}^{t}(t-s)^{(\beta-1) / \alpha} \mathrm{d} s \\
& \leq 2 c_{36}\|g\|_{0,0, t ; X} t^{(\alpha+\beta-1) / \alpha}
\end{aligned}
$$

where $c_{36}=\alpha(\alpha+\beta-1)^{-1} \widetilde{c}_{\alpha, \beta, 0}$. The proof is complete.

Let us now examine the operator $Q_{3}$ defined by (107). To this purpose we need the following result which is proved in [20, Corollary 3.2].

Lemma 27. Let $\delta_{3_{k}} \in(0,1), k=1,2$, be such that $\sigma_{3}=$ $\delta_{3_{1}}+\delta_{3_{2}} \in\left(0,1 / p^{\prime}\right), p \in\left(1 /\left(1-\delta_{3_{1}}\right), \infty\right)$. Then the convolution operator $\mathscr{K}$ defined by (104) maps $C^{\delta_{3_{1}}}\left([0, T] ; X_{1}\right) \times$
$C^{\delta_{3_{2}}}\left([0, T] ; X_{2}\right)$ into $C_{0}^{\sigma_{3}}([0, T] ; X)$, and for every $t \in[0, T]$ satisfies the following estimate:

$$
\begin{aligned}
& \left\|\mathscr{K}\left(g_{3_{1}}, g_{3_{2}}\right)\right\|_{\sigma_{3}, 0, t ; X} \\
& \quad \leq t^{-\sigma_{3}+1 / p^{\prime}} \widetilde{c}_{3}(t)\left\|g_{3_{1}}\right\|_{\delta_{3_{1}}, 0, t ; X_{1}}\left(\int_{0}^{t}\left\|g_{3_{2}}\right\|_{\delta_{3_{2}}, 0, s ; X_{2}}^{p} \mathrm{~d} s\right)^{1 / p} .
\end{aligned}
$$

Here $\widetilde{c}_{3}(t)$ is a nondecreasing function of t depending also on $\delta_{3_{1}}$ and $\delta_{3_{2}}$. Further, in the cases $\delta_{3_{1}} \in(0,1), \delta_{3_{2}}=0$, and $\delta_{3_{1}}=$ $\delta_{3_{2}}=0$, the following estimates hold, respectively, as follows:

$$
\begin{aligned}
& \left\|\mathscr{K}\left(g_{3_{1}}, g_{3_{2}}\right)\right\|_{\delta_{3_{1}}, 0, t ; X} \\
& \quad \leq C_{0} t^{1-\delta_{3_{1}}}\left(1+t^{\delta_{3_{1}}}\right)\left\|g_{3_{1}}\right\|_{\delta_{3_{1}}, 0, t ; X_{1}}\left\|g_{3_{2}}\right\|_{0,0, t ; X_{2}}, \\
& \left\|\mathscr{K}\left(g_{3_{1}}, g_{3_{2}}\right)\right\|_{0,0, t ; X} \leq C_{0} t\left\|g_{3_{1}}\right\|_{0,0, t ; X_{1}}\left\|g_{3_{2}}\right\|_{0,0, t ; X_{2}} \cdot
\end{aligned}
$$

From Lemmas 24 and 27 we obtain the following Lemma 28.

Lemma 28. Let $\alpha$ and $\beta$ be as in Lemma 24. Then, for every $\delta_{3_{1}} \in((3-2 \alpha-\beta) / \alpha, 1)$ and $\delta_{3_{2}} \in(0,1)$ such that $\sigma_{3}=\delta_{3_{1}}+$ $\delta_{3_{2}} \in\left((3-2 \alpha-\beta) / \alpha, 1 / p^{\prime}\right), p \in\left(1 /\left(1-\delta_{3_{1}}\right), \infty\right)$, the operator $Q_{3}$ defined by (107) maps $C^{\delta_{3_{1}}}\left([0, T] ; X_{1}\right) \times C^{\delta_{3_{2}}}\left([0, T] ; X_{2}\right)$ into $C_{0}^{\nu_{3}}([0, T] ; X), \nu_{3}=\left(\alpha \sigma_{3}+2 \alpha+\beta-3\right) / \alpha$, and for every $t \in[0, T]$ satisfies the following estimate:

$$
\begin{aligned}
& \left\|Q_{3}\left(g_{3_{1}}, g_{3_{2}}\right)\right\|_{\gamma_{3}, 0, t ; X} \\
& \leq t^{-\sigma_{3}+1 / p^{\prime}} C_{2}(t) \widetilde{c}_{3}(t)\left\|g_{3_{1}}\right\|_{\delta_{3_{1}}, 0, t ; X_{1}}\left(\int_{0}^{t}\left\|g_{3_{2}}\right\|_{\delta_{3_{2}}, 0, s ; X_{2}}^{p} \mathrm{~d} s\right)^{1 / p} .
\end{aligned}
$$

Proof. First, if $\delta_{3_{1}} \in((3-2 \alpha-\beta) / \alpha, 1)$ and $p \in\left(1 /\left(1-\delta_{3_{1}}\right), \infty\right)$, then $1 / p^{\prime} \in\left(\delta_{3_{1}}, 1\right) \subsetneq((3-2 \alpha-$ $\beta) / \alpha, 1)$. Consequently, the assumption $\sigma_{3}=\delta_{3_{1}}+\delta_{3_{2}} \in((3-$ $\left.2 \alpha-\beta) / \alpha, 1 / p^{\prime}\right), \delta_{3_{2}} \in(0,1)$, makes sense. Now, Lemma 27 yields $\mathscr{K}\left(g_{3_{1}}, g_{3_{2}}\right) \in C_{0}^{\sigma_{3}}([0, T] ; X)$ for any pair $\left(g_{3_{1}}, g_{3_{2}}\right) \in$ $C^{\delta_{3_{1}}}\left([0, T] ; X_{1}\right) \times C^{\delta_{3_{2}}}\left([0, T] ; X_{2}\right)$. Then, recalling that $Q_{3}\left(g_{3_{1}}, g_{3_{2}}\right)=Q_{2} \mathscr{K}\left(g_{3_{1}}, g_{3_{2}}\right)$, the assertion follows from Lemma 24, with $\delta_{2}$ and $g_{2}$ being replaced by $\sigma_{3}$ and $\mathscr{K}\left(g_{3_{1}}, g_{3_{2}}\right)$, respectively. Finally, (134) follows from (117) and (132).

We can now restore the loss of regularity produced by $Q_{2}$.

Proposition 29. Let $5 \alpha+2 \beta>6$ in (H1). Then, for every $\delta_{3} \in((3-2 \alpha-\beta) / \alpha, 1 / 2)$, the operator $Q_{3}$ defined by (107) maps $C^{\delta_{3}}\left([0, T] ; X_{1}\right) \times C^{\delta_{3}}\left([0, T] ; X_{2}\right)$ into $C_{0}^{\delta_{3}}([0, T] ; X)$, and 
for every $t \in[0, T]$ satisfies the following estimate, where $p \in$ $\left(1 /\left(1-2 \delta_{3}\right), \infty\right)$ and $C_{3}(t)=C_{2}(t) \widetilde{c}_{3}(t) \max \left\{1, t^{\left(\alpha \delta_{3}+2 \alpha+\beta-3\right) / \alpha}\right\}$ :

$$
\begin{aligned}
& \left\|Q_{3}\left(g_{3_{1}}, g_{3_{2}}\right)\right\|_{\delta_{3}, 0, t ; X} \\
& \quad \leq t^{1-2 \delta_{3}-1 / p} C_{3}(t)\left\|g_{3_{1}}\right\|_{\delta_{3}, 0, t ; X_{1}}\left(\int_{0}^{t}\left\|g_{3_{2}}\right\|_{\delta_{3}, 0, s ; X_{2}}^{p} \mathrm{~d} s\right)^{1 / p} .
\end{aligned}
$$

Proof. Let $\delta_{3} \in((3-2 \alpha-\beta) / \alpha, 1 / 2)$ and let $p \in(1 /(1-$ $\left.\left.2 \delta_{3}\right), \infty\right) \subsetneq\left(1 /\left(1-\delta_{3}\right), \infty\right)$. Then, $2 \delta_{3} \in((6-4 \alpha-$ $\left.2 \beta) / \alpha, 1 / p^{\prime}\right) \subseteq\left((3-2 \alpha-\beta) / \alpha, 1 / p^{\prime}\right)$. We are thus in position to apply Lemma 28 with $\delta_{3_{1}}=\delta_{3_{2}}=\delta_{3}$ from which we deduce that $Q_{3}$ maps $C^{\delta_{3}}\left([0, T] ; X_{1}\right) \times C^{\delta_{3}}\left([0, T] ; X_{2}\right)$ into $C_{0}^{\nu_{3}}([0, T] ; X), \nu_{3}=\left(2 \alpha \delta_{3}+2 \alpha+\beta-3\right) / \alpha$. But, since our choice for $\delta_{3}$ implies $\nu_{3}>\delta_{3}$, we a fortiori have the fact that $Q_{3}$ maps $C^{\delta_{3}}\left([0, T] ; X_{1}\right) \times C^{\delta_{3}}\left([0, T] ; X_{2}\right)$ into $C_{0}^{\delta_{3}}([0, T] ; X)$. Finally, (135) follows from (134) and the estimate $\|g\|_{\gamma, 0, t ; X} \leq$ $\max \left\{1, t^{\delta-\gamma}\right\}\|g\|_{\delta, 0, t ; X}, g \in C^{\delta}([0, T] ; X), \delta \geq \gamma$.

The next Lemma 30 concerns the operator $Q_{4}$. Its proof is similar to that of Lemma 24, but with the essential difference that the presence of $y \in Y_{\gamma}^{p}$ allows us to use estimate (79) in place of (14). As a consequence and provided to choose $\gamma$ large enough, we will achieve a better result in which any loss of regularity is observed.

Lemma 30. Let $2 \alpha+\beta>2$ in (H1) and $r \in[1, \infty]$. Then, for every $\delta_{4} \in(0,1)$ and $\gamma \in(3-2 \alpha-\beta, 1)$ the operator $Q_{4}$ defined by (108) maps $C^{\delta_{4}}([0, T] ; \mathbf{C}) \times Y_{\gamma}^{r}, Y_{\gamma}^{r} \in\left\{(X, \mathscr{D}(A))_{\gamma, r}, X_{A}^{\gamma, r}\right\}$, into $C_{0}^{\delta_{4}}([0, T] ; X)$, and for every $t \in[0, T]$ satisfies the following estimate:

$$
\left\|Q_{4}\left(g_{4}, y\right)\right\|_{\delta_{4}, 0, t ; X} \leq C_{4}(t) t^{(2 \alpha+\beta+\gamma-3) / \alpha}\left|g_{4}\right|_{\delta_{4}, 0, t ; \mathbf{C}}\|y\|_{Y_{\gamma}^{r}} .
$$

Here $C_{4}(t)$ is a nondecreasing function of t depending on $\alpha, \beta$, $\delta_{4}, \gamma$ and $r$.

Proof. Let $t \in[0, T], g_{4} \in C^{\delta_{4}}([0, T] ; \mathbf{C}), \delta_{4} \in(0,1)$, and $y \in Y_{\gamma}^{r}, \gamma \in(3-2 \alpha-\beta, 1), r \in[1, \infty]$. As in the proof of Lemma 24 we set $\bar{\alpha}=(1-\alpha) / \alpha$ and we observe that, since $2 \alpha+\beta>2$ implies $\alpha \in(1 / 2,1]$, here $\bar{\alpha} \in[0,1)$. Furthermore, we denote by $\sigma_{\alpha, \beta, \gamma}$ the number $(2 \alpha+\beta+\gamma-3) / \alpha \in(0,1)$, so that the exponents $(\beta+\gamma-2) / \alpha$ and $(\beta+\gamma-3) / \alpha$ appearing in (79) with $\theta=1$ and $\theta=2$ may be rewritten, as $\sigma_{\alpha, \beta, \gamma}+\bar{\alpha}-1$ and $\sigma_{\alpha, \beta, \gamma}-2$, respectively. Then, using (79) with $\theta=1$, we obtain

$$
\begin{aligned}
& \left\|\left[Q_{4}\left(g_{4}, y\right)\right](\tau)\right\|_{X} \\
& \quad \leq c_{22}\left|g_{4}\right|_{\delta_{4}, 0, \tau ; \mathrm{C}}\|y\|_{Y_{\gamma}^{r}} \int_{0}^{\tau}(\tau-s)^{\delta_{4}+\sigma_{\alpha, \beta, \gamma}+\bar{\alpha}-1} \mathrm{~d} s \\
& \quad=c_{37}\left|g_{4}\right|_{\delta_{4}, 0, \tau ; \mathrm{C}}\|y\|_{Y_{\gamma}^{r}} \tau^{\delta_{4}+\sigma_{\alpha, \beta, \gamma}+\bar{\alpha}}, \quad \forall \tau \in[0, t],
\end{aligned}
$$

where $c_{37}=c_{22}\left(\delta_{4}+\sigma_{\alpha, \beta, \gamma}+\bar{\alpha}\right)^{-1}$. Hence, taking the supremum with respect to $\tau \in[0, t]$, one has

$$
\left\|Q_{4}\left(g_{4}, y\right)\right\|_{0,0, t ; X} \leq c_{37}\left|g_{4}\right|_{\delta_{4}, 0, t ; \mathbf{C}}\|y\|_{Y_{\gamma}^{r}} t^{\delta_{4}+\sigma_{\alpha, \beta, \gamma}+\bar{\alpha}} .
$$

Now, let $\left(\right.$ since $\left[Q_{4}\left(g_{4}, y\right)\right](0)=0$, the case $t_{1}=0$ follows from (137) with $\left.\tau=t_{2}\right) 0<t_{1}<t_{2} \leq t$. We have $\left[Q_{4}\left(g_{4}, y\right)\right]\left(t_{2}\right)-\left[Q_{4}\left(g_{4}, y\right)\right]\left(t_{1}\right)=\sum_{k=1}^{3} J_{k ; t_{1}, t_{2}, g_{4} y}$, the $J_{k ; t_{1}, t_{2}, g}$ 's, $g:[0, T] \rightarrow X$, being as in (120). Using (13) with $(s, t, \theta)=\left(t_{1}-s, t_{2}-s, 1\right), s \in\left(0, t_{1}\right)$, and (79) with $\theta=2$, and letting $\left(c_{38}, c_{39}\right)=\left(c_{22}\left(1-\delta_{4}\right)^{-1}, c_{38} \delta_{4}^{-1}\right)$, we get

$$
\begin{aligned}
\left\|J_{1 ; t_{1}, t_{2}, g_{4} y} y\right\|_{X} & \\
\leq & c_{22}\left|g_{4}\right|_{\delta_{4}, 0, t_{1} ; \mathbf{C}}\|y\|_{Y_{\gamma}^{r}} \\
& \times \int_{0}^{t_{1}}\left[\int_{t_{1}-s}^{t_{2}-s} \xi \sigma_{\alpha, \beta, \gamma}^{\sigma_{3}-2} \mathrm{~d} \xi\right]\left(t_{1}-s\right)^{\delta_{4}} \mathrm{~d} s \\
\leq & c_{22}\left|g_{4}\right|_{\delta_{4}, 0, t_{1} ; \mathbf{C}}\|y\|_{Y_{\gamma}^{r}} \int_{0}^{t_{1}}\left[\int_{t_{1}-s}^{t_{2}-s} \xi^{\delta_{4}+\sigma_{\alpha, \beta, \gamma}-2} \mathrm{~d} \xi\right] \mathrm{d} s \\
\leq & c_{22}\left|g_{4}\right|_{\delta_{4}, 0, t_{1} ; \mathbf{C}}\|y\|_{Y_{\gamma}^{r}} \\
& \times \int_{0}^{t_{1}}\left(t_{2}-s\right)^{\sigma_{\alpha, \beta, \gamma}}\left[\int_{t_{1}-s}^{t_{2}-s} \xi^{\delta_{4}-2} \mathrm{~d} \xi\right] \mathrm{d} s \\
\leq & c_{38}\left|g_{4}\right|_{\delta_{4}, 0, t_{2} ; \mathbf{C}}\|y\|_{Y_{\gamma}^{r}} t_{2}^{\sigma_{\alpha, \beta, \gamma}} \\
& \times \int_{0}^{t_{1}}\left[\left(t_{1}-s\right)^{\delta_{4}-1}-\left(t_{2}-s\right)^{\delta_{4}-1}\right] \mathrm{d} s \\
= & c_{39}\left|g_{4}\right|_{\delta_{4}, 0, t_{2} ; \mathbf{C}}\|y\|_{Y_{\gamma}^{r}} t_{2}^{\sigma_{\alpha, \beta, \gamma}}\left[t_{1}^{\delta_{4}}+\left(t_{2}-t_{1}\right)^{\delta_{4}}-t_{2}^{\delta_{4}}\right] \\
\leq & c_{39}\left|g_{4}\right|_{\delta_{4}, 0, t_{2} ; \mathbf{C}}\|y\|_{Y_{\gamma}^{r}} t_{2}^{\sigma_{\alpha, \beta, \gamma}}\left(t_{2}-t_{1}\right)^{\delta_{4}} .
\end{aligned}
$$

Now, let us examine $J_{k ; t_{1}, t_{2}, g_{4} y}, k=2,3$. First, using (79) with $\theta=1$, we find

$\| J_{2 ; t_{1}, t_{2}, g_{4} y \|_{X}}$

$$
\begin{aligned}
& \leq c_{22}\left|g_{4}\right|_{\delta_{4}, 0, t_{2} ; \mathbf{C}}\|y\|_{Y_{\gamma}^{r}}\left[\int_{0}^{t_{1}}\left(t_{2}-s\right)^{\sigma_{\alpha, \beta, \gamma}+\bar{\alpha}-1} \mathrm{~d} s\right]\left(t_{2}-t_{1}\right)^{\delta_{4}} \\
& =c_{40}\left|g_{4}\right|_{\delta_{4}, 0, t_{2} ; \mathbf{C}}\|y\|_{Y_{\gamma}^{r}}\left[t_{2}^{\sigma_{\alpha, \beta, \gamma}+\bar{\alpha}}-\left(t_{2}-t_{1}\right)^{\sigma_{\alpha, \beta, \gamma}+\bar{\alpha}}\right]\left(t_{2}-t_{1}\right)^{\delta_{4}} \\
& \leq c_{40}\left|g_{4}\right|_{\delta_{4}, 0, t_{2} ; \mathbf{C}}\|y\|_{Y_{\gamma}^{r}} t_{2}^{\sigma_{\alpha, \beta, \gamma}+\bar{\alpha}}\left(t_{2}-t_{1}\right)^{\delta_{4}} .
\end{aligned}
$$

Instead, the same computations made to derive (137) yield

$$
\begin{aligned}
& \left\|J_{3 ; t_{1}, t_{2}, g_{4} y}\right\|_{X} \\
& \leq c_{22}\left|g_{4}\right|_{\delta_{4}, 0, t_{2} ;}\|y\|_{Y_{\gamma}^{r}} \int_{t_{1}}^{t_{2}}\left(t_{2}-s\right)^{\delta_{4}+\sigma_{\alpha, \beta, \gamma}+\bar{\alpha}-1} \mathrm{~d} s \\
& \quad=c_{37}\left|g_{4}\right|_{\delta_{4}, 0, t_{2} ;}\|y\|_{Y_{\gamma}^{r}}\left(t_{2}-t_{1}\right)^{\delta_{4}+\sigma_{\alpha, \beta, \gamma}+\bar{\alpha}} .
\end{aligned}
$$

From (139)-(141) and $\left\|\left[Q_{4}\left(g_{4}, y\right)\right]\left(t_{2}\right)-\left[Q_{4}\left(g_{4}, y\right)\right]\left(t_{1}\right)\right\|_{X} \leq$ $\sum_{k=1}^{3}\left\|J_{k ; t_{1}, t_{2}, g_{4} y}\right\|_{X}$, it follows that

$$
\begin{aligned}
& \left\|\left[Q_{4}\left(g_{4}, y\right)\right]\left(t_{2}\right)-\left[Q_{4}\left(g_{4}, y\right)\right]\left(t_{1}\right)\right\|_{X} \\
& \quad \leq \widetilde{c}_{4}(t) t^{\sigma_{\alpha, \beta, \gamma}}\left|g_{4}\right|_{\delta_{4}, 0, t ; C}\|y\|_{Y_{\gamma}^{r}}\left(t_{2}-t_{1}\right)^{\delta_{4}},
\end{aligned}
$$


where $\widetilde{c}_{4}(t)=c_{39}+\left(c_{37}+c_{40}\right) t^{\bar{\alpha}}$. Finally, summing up (138) and (142) we get (136) with $C_{4}(t)=c_{37} t^{\delta_{4}+\bar{\alpha}}+\widetilde{c}_{4}(t)$. The proof is complete.

Remark 31. Notice that if $Y_{\gamma}^{r}=X_{A}^{\gamma, r}$, then in order to be sure that the conclusions of Lemma 30 hold with $y$ which really belongs to some intermediate space between $X$ and $\mathscr{D}(A)$ we have to choose $\gamma \in(3-2 \alpha-\beta, \beta)$. This is possible, provided that the stronger assumption $2 \alpha+\beta>3-\beta \geq 2$ is satisfied. Otherwise, if $2 \alpha+\beta \in(2,3-\beta], \beta<1$, then $\gamma \in(3-2 \alpha-\beta, 1) \varsubsetneqq$ $[\beta, 1)$ and $y$ may be contained in $\mathscr{D}(A)$.

Finally, for the operator $Q_{5}$ we have the following result. Again a loss of regularity is exhibited, even though of an amount smaller than that in Lemma 24 (cf. Remark 33).

Lemma 32. Let $2 \alpha+\beta>2$ in (H1). Then, for every $\delta_{5} \in$ $((2-\alpha-\beta) / \alpha, 1)$, the operator $Q_{5}$ defined by (109) maps $C_{0}^{\delta_{5}}([0, T] ; X)$ into $C_{0}^{\nu_{5}}([0, T] ; X), \nu_{5}=\left(\alpha \delta_{5}+\alpha+\beta-2\right) / \alpha \in$ $\left(0, \delta_{5}\right]$, and for every $t \in[0, T]$ satisfies the following estimate:

$$
\left\|Q_{5} g_{5}\right\|_{\nu_{5}, 0, t ; X} \leq C_{5}(t)\left|g_{5}\right|_{\delta_{5}, 0, t ; X}
$$

Here $C_{5}(t)$ is a nondecreasing function of $t$ depending also on $\alpha, \beta$, and $\delta_{5}$.

Proof. Let $g_{5} \in C_{0}^{\delta_{5}}([0, T] ; X), \delta_{5} \in((2-\alpha-\beta) / \alpha, 1)$, and $v_{5}=\left(\alpha \delta_{5}+\alpha+\beta-2\right) / \alpha \in\left(0, \delta_{5}\right]$. We still let $\bar{\alpha}=(1-\alpha) / \alpha$ and as in Lemma 30 we have $\bar{\alpha} \in[0,1)$. Further, observe that $\delta_{5}+(\beta-1) / \alpha=\nu_{5}+\bar{\alpha} \in\left(0, \delta_{5}\right]$. Let $t \in[0, T]$. Then, using (14) and $g_{5}(0)=0$, we get

$$
\begin{aligned}
& \left\|Q_{5} g_{5}\right\|_{0,0, t ; X} \\
& \quad \leq \sup _{\tau \in[0, t]}\left[\widetilde{c}_{\alpha, \beta, 0} \tau^{(\beta-1) / \alpha}+1\right]\left|g_{5}\right|_{\delta_{5}, 0, \tau ; X} \tau^{\delta_{5}} \\
& \quad \leq\left[\widetilde{c}_{\alpha, \beta, 0}+t^{(1-\beta) / \alpha}\right]\left|g_{5}\right|_{\delta_{5}, 0, t ; X} t^{\nu_{5}+\bar{\alpha}}
\end{aligned}
$$

Now, let (since $\left[Q_{5} g_{5}\right](0)=0$, the case $t_{1}=0$ follows from (144) and $\left.\left\|\left[Q_{5} g_{5}\right]\left(t_{2}\right)\right\|_{X} \leq\left\|Q_{5} g_{5}\right\|_{0,0, t_{2} ; X}\right) 0<t_{1}<t_{2} \leq t$. We have $\left[Q_{5} g_{5}\right]\left(t_{2}\right)-\left[Q_{5} g_{5}\right]\left(t_{1}\right)=\sum_{k=1}^{3} U_{k ; t_{1}, t_{2}, g_{5}}$, where for a function $g:[0, T] \rightarrow X$ we let

$$
\begin{gathered}
U_{1 ; t_{1}, t_{2}, g}:=\mathrm{e}^{t_{2} A}\left[g\left(t_{2}\right)-g\left(t_{1}\right)\right], \\
U_{2 ; t_{1}, t_{2}, g}:=\left(\mathrm{e}^{t_{2} A}-\mathrm{e}^{t_{1} A}\right) g\left(t_{1}\right), \\
U_{3 ; t_{1}, t_{2}, g}:=g\left(t_{1}\right)-g\left(t_{2}\right) .
\end{gathered}
$$

First, since $t_{2}^{(\beta-1) / \alpha} \leq\left(t_{2}-t_{1}\right)^{(\beta-1) / \alpha}$ for every $\beta \in(0,1]$, we deduce that

$$
\begin{aligned}
\left\|U_{1 ; t_{1}, t_{2}, g_{5}}\right\|_{X} & \leq \widetilde{c}_{\alpha, \beta, 0} t_{2}^{(\beta-1) / \alpha}\left|g_{5}\right|_{\delta_{5}, 0, t_{2} ; X}\left(t_{2}-t_{1}\right)^{\delta_{5}} \\
& \leq \widetilde{c}_{\alpha, \beta, 0}\left|g_{5}\right|_{\delta_{5}, 0, t_{2} ; X}\left(t_{2}-t_{1}\right)^{\nu_{5}+\bar{\alpha}} .
\end{aligned}
$$

As far as $U_{2 ; t_{1}, t_{2}, g_{5}}$ is concerned, instead, rewriting $\mathrm{e}^{t_{2} A}-\mathrm{e}^{t_{1} A}$ as $-\int_{t_{1}}^{t_{2}}\left[(-A)^{1}\right]^{\circ} \mathrm{e}^{r A} \mathrm{~d} r$ and using both $g_{5}(0)=0$ and $\left(\alpha \delta_{5}+\right.$ $\beta-2) / \alpha=\nu_{5}-1$, it follows that

$$
\begin{aligned}
\left\|U_{2 ; t_{1}, t_{2}, g_{5}}\right\|_{X} & \leq \widetilde{c}_{\alpha, \beta, 1}\left|g_{5}\right|_{\delta_{5}, 0, t_{1} ; X} t_{1}^{\delta_{5}} \int_{t_{1}}^{t_{2}} r^{(\beta-2) / \alpha} \mathrm{d} r \\
& \leq \widetilde{c}_{\alpha, \beta, 1}\left|g_{5}\right|_{\delta_{5}, 0, t_{1} ; X} \int_{t_{1}}^{t_{2}} r^{\nu_{5}-1} \mathrm{~d} r \\
& \leq \widetilde{c}_{\alpha, \beta, 1} v_{5}^{-1}\left|g_{5}\right|_{\delta_{5}, 0, t_{1} ; X}\left(t_{2}^{\nu_{5}}-t_{1}^{\nu_{5}}\right) \\
& \leq \widetilde{c}_{\alpha, \beta, 1} v_{5}^{-1}\left|g_{5}\right|_{\delta_{5}, 0, t_{1} ; X}\left(t_{2}-t_{1}\right)^{\nu_{5}} .
\end{aligned}
$$

Then, since $\left\|U_{3 ; t_{1}, t_{2}, g_{5}}\right\|_{X} \leq\left|g_{5}\right|_{\delta_{5}, 0, t_{2} ; X}\left(t_{2}-t_{1}\right)^{\delta_{5}}$, from (146) and (147) we find

$$
\begin{aligned}
& \left\|\left[Q_{5} g_{5}\right]\left(t_{2}\right)-\left[Q_{5} g_{5}\right]\left(t_{1}\right)\right\|_{X} \\
& \quad \leq \sum_{k=1}^{3}\left\|U_{k ; t_{1}, t_{2}, g_{5}}\right\|_{X} \leq \widetilde{c}_{5}(t)\left|g_{5}\right|_{\delta_{5}, 0, t ; X}\left(t_{2}-t_{1}\right)^{\nu_{5}},
\end{aligned}
$$

where $\widetilde{c}_{5}(t)=\widetilde{c}_{\alpha, \beta, 0} t^{\bar{\alpha}}+\widetilde{c}_{\alpha, \beta, 1} v_{5}^{-1}+t^{\delta_{5}-v_{5}}$. Summing up (144) and (148) we obtain (143) with $C_{5}(t)=\left[\widetilde{c}_{\alpha, \beta, 0}+t^{(1-\beta) / \alpha}\right] t^{\nu_{5}+\bar{\alpha}}+\widetilde{c}_{5}(t)$. This completes the proof.

Remark 33. Thus, with the exception of $\beta=1, Q_{5}$ produces a loss of regularity equal to $\delta_{5}-\nu_{5}=(2-\alpha-\beta) / \alpha \leq(3-2 \alpha-$ $\beta) / \alpha$. In this sense $Q_{5}$ behaves better than $Q_{2}$.

Remark 34. Notice that, under the weaker assumptions $\alpha+$ $\beta>1$ and $g_{5} \in C([0, T] ; X),(86)$ with $x=g_{5}(t), t \in[0, T]$, yields $A^{-1}\left[Q_{5} g_{5}\right](t)=\left[Q_{5}\left(A^{-1} g_{5}\right)\right](t)=\int_{0}^{t} \mathrm{e}^{(t-s) A} g_{5}(t) \mathrm{d} s$.

Similarly as we have done in Proposition 29 for restoring the loss of regularity produced by $Q_{2}$, we now show how Lemma 27 allows to restore that produced by $Q_{5}$. We begin with the following version of Lemma 28 relative to $Q_{6}$, and which is obtained combining Lemma 27 with Lemma 32 instead of Lemma 24.

Lemma 35. Let $\alpha$ and $\beta$ be as in Lemma 32. Then, for every $\delta_{6_{1}} \in((2-\alpha-\beta) / \alpha, 1)$ and $\delta_{6_{2}} \in(0,1)$ such that $\sigma_{6}=\delta_{6_{1}}+\delta_{6_{2}} \in$ $\left((2-\alpha-\beta) / \alpha, 1 / p^{\prime}\right), p \in\left(1 /\left(1-\delta_{6_{1}}\right), \infty\right)$, the operator $Q_{6}$ defined by (110) maps $C^{\delta_{6_{1}}}\left([0, T] ; X_{1}\right) \times C^{\delta_{6_{2}}}\left([0, T] ; X_{2}\right)$ into $C_{0}^{v_{6}}([0, T] ; X), \nu_{6}=\left(\alpha \sigma_{6}+\alpha+\beta-2\right) / \alpha$, and for every $t \in[0, T]$ satisfies the following estimate:

$$
\begin{aligned}
& \left\|Q_{6}\left(g_{6_{1}}, g_{6_{2}}\right)\right\|_{\nu_{6}, 0, t ; X} \\
& \leq t^{-\sigma_{6}+1 / p^{\prime}} C_{5}(t) \widetilde{c}_{3}(t)\left\|g_{6_{1}}\right\|_{\delta_{6_{1}}, 0, t ; X_{1}}\left(\int_{0}^{t}\left\|g_{6_{2}}\right\|_{\delta_{\sigma_{2}}, 0, s ; X_{2}}^{p} \mathrm{~d} s\right)^{1 / p} .
\end{aligned}
$$

Proof. First, if $\delta_{6_{1}} \in((2-\alpha-\beta) / \alpha, 1)$ and $p \in\left(1 /\left(1-\delta_{6_{1}}\right), \infty\right)$, then $1 / p^{\prime} \in\left(\delta_{6_{1}}, 1\right) \subsetneq((2-\alpha-\beta) / \alpha, 1)$. Consequently, the assumption $\sigma_{6}=\delta_{6_{1}}+\delta_{6_{2}} \in\left((2-\alpha-\beta) / \alpha, 1 / p^{\prime}\right)$ makes sense, 
provided to choose $\delta_{6_{2}} \in(0,1)$ small enough. Lemma 27 then yields $\mathscr{K}\left(g_{6_{1}}, g_{6_{2}}\right) \in C_{0}^{\sigma_{6}}([0, T] ; X)$ for any pair $\left(g_{6_{1}}, g_{6_{2}}\right) \in$ $C^{\delta_{6_{1}}}\left([0, T] ; X_{1}\right) \times C^{\delta_{6_{2}}}\left([0, T] ; X_{2}\right)$. Then, since $Q_{6}\left(g_{6_{1}}, g_{6_{2}}\right)=$ $Q_{5} \mathscr{K}\left(g_{6_{1}}, g_{6_{2}}\right)$, the assertion follows from Lemma 32, with the pair $\left(\delta_{5}, g_{5}\right)$ being replaced by $\left(\sigma_{6}, \mathscr{K}\left(g_{6_{1}}, g_{6_{2}}\right)\right)$. Finally, (149) follows from (143) and (132).

From Lemma 35 we obtain the analogous of Proposition 29 for $Q_{6}$.

Proposition 36. Let $3 \alpha+2 \beta>4$ in (H1). Then, for every $\delta_{6} \in((2-\alpha-\beta) / \alpha, 1 / 2)$, the operator $Q_{6}$ defined by (110) maps $C^{\delta_{6}}\left([0, T] ; X_{1}\right) \times C^{\delta_{6}}\left([0, T] ; X_{2}\right)$ into $C_{0}^{\delta_{6}}([0, T] ; X)$, and for every $t \in[0, T]$ satisfies the following estimate, where $p \in$ $\left(1 /\left(1-2 \delta_{6}\right), \infty\right)$ and $C_{6}(t)=C_{5}(t) \widetilde{c}_{3}(t) \max \left\{1, t^{\left(\alpha \delta_{6}+\alpha+\beta-2\right) / \alpha}\right\}$ :

$$
\begin{aligned}
& \left\|Q_{6}\left(g_{6_{1}}, g_{6_{2}}\right)\right\|_{\delta_{6}, 0, t ; X} \\
& \quad \leq t^{1-2 \delta_{6}-1 / p} C_{6}(t)\left\|g_{6_{1}}\right\|_{\delta_{6}, 0, t ; X_{1}}\left(\int_{0}^{t}\left\|g_{6_{2}}\right\|_{\delta_{6}, 0, s ; X_{2}}^{p} \mathrm{~d} s\right)^{1 / p} .
\end{aligned}
$$

Proof. Let $\delta_{6} \in((2-\alpha-\beta) / \alpha, 1 / 2)$ and $p \in\left(1 /\left(1-2 \delta_{6}\right), \infty\right) \varsubsetneqq$ $\left(1 /\left(1-\delta_{6}\right), \infty\right)$. Then, $2 \delta_{6} \in\left((4-2 \alpha-2 \beta) / \alpha, 1 / p^{\prime}\right) \subseteq$ $\left((2-\alpha-\beta) / \alpha, 1 / p^{\prime}\right)$ and we can apply Lemma 35 with $\delta_{6_{k}}=\delta_{6}$, $k=1,2$. We thus deduce that $Q_{6}$ maps $C^{\delta_{6}}\left([0, T] ; X_{1}\right) \times$ $C^{\delta_{6}}\left([0, T] ; X_{2}\right)$ into $C_{0}^{\nu_{6}}([0, T] ; X), \nu_{6}=\left(2 \alpha \delta_{6}+\alpha+\beta-2\right) / \alpha$. But, since $\delta_{6}>(2-\alpha-\beta) / \alpha$ implies $\nu_{6}>\delta_{6}$, we a fortiori have the fact that $Q_{6}$ maps $C^{\delta_{6}}\left([0, T] ; X_{1}\right) \times C^{\delta_{6}}\left([0, T] ; X_{2}\right)$ into $C_{0}^{\delta_{6}}([0, T] ; X)$. Finally, (150) follows from (149) and $\left\|Q_{6}\left(g_{6_{1}}, g_{6_{2}}\right)\right\|_{\delta_{6}, 0, t ; X} \leq \max \left\{1, t^{v_{6}-\delta_{6}}\right\}\left\|Q_{6}\left(g_{6_{1}}, g_{6_{2}}\right)\right\|_{v_{6}, 0, t ; X}$.

In Section 6 we will also encounter $Q_{5}$ acting on functions which enjoy some space regularity, that is, functions $g_{5}$ which are Hölder continuous in time with values on $Y_{\gamma}^{r} \in$ $\left\{(X, \mathscr{D}(A))_{\gamma, r}, X_{A}^{\gamma, r}\right\}$. In this case Lemma 32 can be refined, and the loss of regularity produced by $Q_{5}$ is naturally restored by the additional condition of space regularity on $g_{5}$. In some sense, the forthcoming Corollary 38 is the analogous of Lemma 30, where the function $g_{4} y$ involved in the definition of $Q_{4}\left(g_{4}, y\right)$ (cf. (108)) was of class $C^{\delta_{4}}\left([0, T] ; Y_{\gamma}^{r}\right)$.

Lemma 37. Let $\alpha+\beta>1$ in (H1) and $Y_{\gamma}^{r} \in\left\{(X, \mathscr{D}(A))_{\gamma, r}\right.$, $\left.X_{A}^{\gamma, r}\right\}, \gamma \in(2-\alpha-\beta, 1), r \in[1, \infty]$. Then, for every $\delta_{5} \in$ $(0,(\alpha+\beta+\gamma-2) / \alpha]$, the operator $Q_{5}$ defined by (109) maps $C^{\delta_{5}}\left([0, T] ; Y_{\gamma}^{r}\right)$ into $C_{0}^{\delta_{5}}([0, T], X)$, and for every $t \in[0, T]$ satisfies the following estimate:

$$
\left\|Q_{5} g_{5}\right\|_{\delta_{5}, 0, t ; X} \leq c_{41} t^{\left(\alpha+\beta+\gamma-2-\alpha \delta_{5}\right) / \alpha}\left(2 t^{\delta_{5}}+1\right)\left\|g_{5}\right\|_{\delta_{5}, 0, t ; Y_{\gamma}^{r}} .
$$

Here $c_{41}$ is a positive constant depending on $\alpha, \beta, \gamma$, and $r$.

Proof. Let $\gamma \in(2-\alpha-\beta, 1) \subseteq(1-\beta, 1)$ and let $\chi_{\alpha, \beta, \gamma}$ be the number $(\alpha+\beta+\gamma-2) / \alpha \in(0,1)$, so that the exponent $(\beta+\gamma-2) / \alpha$ in (79) with $\theta=1$ is equal to $\chi_{\alpha, \beta, \gamma}-1$.
Let $g_{5} \in C^{\delta_{5}}\left([0, T] ; Y_{\gamma}^{r}\right), \delta_{5} \in\left(0, \chi_{\alpha, \beta, \gamma}\right], r \in[1, \infty]$. Since $\left[Q_{5} g_{5}\right](0)=0$, we assume that $t \in(0, T]$ and we observe that, due to Propositions 6 and $12,\left[Q_{5} g_{5}\right](t)$ is rewritten as follows:

$$
\begin{aligned}
{\left[Q_{5} g_{5}\right](t) } & =\left[\mathrm{e}^{t A}-I\right] g_{5}(t)=\lim _{\varepsilon \rightarrow 0^{+}}\left[\mathrm{e}^{t A}-\mathrm{e}^{\varepsilon A}\right] g_{5}(t) \\
& =\lim _{\varepsilon \rightarrow 0^{+}} \int_{\varepsilon}^{t} D_{s} \mathrm{e}^{s A} g_{5}(t) \mathrm{d} s \\
& =-\lim _{\varepsilon \rightarrow 0^{+}} \int_{\varepsilon}^{t}\left[(-A)^{1}\right]^{\circ} \mathrm{e}^{s A} g_{5}(t) \mathrm{d} s \\
& =-\int_{0}^{t}\left[(-A)^{1}\right]^{\circ} \mathrm{e}^{s A} g_{5}(t) \mathrm{d} s .
\end{aligned}
$$

Indeed, for every $\varepsilon \in[0, t)$ and $x \in Y_{\gamma}^{r}$, (79) with $\theta=1$ yields

$$
\begin{aligned}
& \left\|\int_{\varepsilon}^{t}\left[(-A)^{1}\right]^{\circ} \mathrm{e}^{s A} x \mathrm{~d} s\right\|_{X} \\
& \quad \leq c_{22}\|x\|_{Y_{\gamma}^{r}} \int_{\varepsilon}^{t} s^{\chi_{\alpha, \beta, \gamma}-1} \mathrm{~d} s \leq c_{41}\|x\|_{Y_{\gamma}^{r}}(t-\varepsilon)^{\chi_{\alpha, \beta, \gamma},}
\end{aligned}
$$

where $c_{41}=c_{22} \chi_{\alpha, \beta, \gamma}^{-1}$. From (152) and (153) with $(\varepsilon, t, x)=$ $\left(0, \tau, g_{5}(\tau)\right)$ we thus get

$$
\begin{aligned}
\left\|Q_{5} g_{5}\right\|_{0,0, t ; X} & =\sup _{\tau \in[0, t]}\left\|\left[Q_{5} g_{5}\right](\tau)\right\|_{X} \\
& \leq c_{41}\left\|g_{5}\right\|_{0,0, t ; Y_{\gamma}^{r}} t^{\chi_{\alpha, \beta, \gamma}} .
\end{aligned}
$$

Now, let $0 \leq t_{1}<t_{2} \leq t$. From (152) it follows that $\left[Q_{5} g_{5}\right]\left(t_{2}\right)-$ $\left[Q_{5} g_{5}\right]\left(t_{1}\right)=-\sum_{k=1}^{2} V_{k ; t_{1}, t_{2}, g_{5}}$, where for every function $g$ : $[0, T] \rightarrow Y_{\gamma}^{p}$ we have set

$$
\begin{gathered}
V_{1 ; t_{1}, t_{2}, g}:=\int_{0}^{t_{1}}\left[(-A)^{1}\right]^{\circ} \mathrm{e}^{s A}\left[g\left(t_{2}\right)-g\left(t_{1}\right)\right] \mathrm{d} s, \\
V_{2 ; t_{1}, t_{2}, g}:=\int_{t_{1}}^{t_{2}}\left[(-A)^{1}\right]^{\circ} \mathrm{e}^{s A} g\left(t_{2}\right) \mathrm{d} s .
\end{gathered}
$$

Hence, using (153) with the triplet $(\varepsilon, t, x)$ being replaced by $\left(0, t_{1}, g_{5}\left(t_{2}\right)-g_{5}\left(t_{1}\right)\right)$ and $\left(t_{1}, t_{2}, g_{5}\left(t_{2}\right)\right)$, respectively, we deduce that

$$
\begin{gathered}
\left\|V_{1 ; t_{1}, t_{2}, g_{5}}\right\|_{X} \leq c_{41}\left|g_{5}\right|_{\delta_{5}, 0, t_{2} ; Y_{\gamma}} t_{1}^{\chi_{\alpha, \beta, \gamma}}\left(t_{2}-t_{1}\right)^{\delta_{5}}, \\
\left\|V_{2 ; t_{1}, t_{2}, g_{5}}\right\|_{X} \leq c_{41}\left\|g_{5}\right\|_{0,0, t_{2} ; Y_{\gamma}^{r}}\left(t_{2}-t_{1}\right)^{\chi_{\alpha, \beta, \gamma}} .
\end{gathered}
$$

As a consequence, since $\delta_{5} \in\left(0, \chi_{\alpha, \beta, \gamma}\right]$,

$$
\begin{aligned}
& \left\|\left[Q_{5} g_{5}\right]\left(t_{2}\right)-\left[Q_{5} g_{5}\right]\left(t_{1}\right)\right\|_{X} \\
& \leq c_{41} t^{\chi \alpha, \beta, \gamma}-\delta_{5}\left(t^{\delta_{5}}+1\right)\left\|g_{5}\right\|_{\delta_{5}, 0, t ; Y_{\gamma}^{r}}\left(t_{2}-t_{1}\right)^{\delta_{5}} .
\end{aligned}
$$

Summing up (154) and (157), we obtain (151). The proof is complete.

Since in Lemma 37 it is not required that $g_{5}(0)=0$, the special case of the constant function $g_{5}(t)=x \in Y_{\gamma}^{p}, t \in$ $[0, T]$, is admissible, and we obtain the following result. 
Corollary 38. Let $\alpha, \beta$, and $Y_{\gamma}^{r}$ be as in Lemma 37, and let $x \in$ $Y_{\gamma}^{r}, \gamma \in(2-\alpha-\beta, 1)$, and $r \in[1, \infty]$. Then, for every $\delta_{7} \in$ $(0,(\alpha+\beta+\gamma-2) / \alpha]$, the function $\left[Q_{7} x\right](\cdot):=\left(e^{\cdot A}-I\right) x$ belongs to $C_{0}^{\delta_{7}}([0, T] ; X)$, and for every $t \in[0, T]$ satisfies the estimate

$$
\left\|Q_{7} x\right\|_{\delta_{7}, 0, t ; X} \leq c_{41} t^{\left(\alpha+\beta+\gamma-2-\alpha \delta_{7}\right) / \alpha}\left(t^{\delta_{7}}+1\right) c_{41}\|x\|_{Y_{\gamma}^{r}} .
$$

Proof. Let $g_{5}(t)=x$ in the proof of Lemma 37, and observe that $V_{1, t_{1}, t_{2}, g_{5}}$ reduces to the zero element of $X$. Estimate (158) then follows from (154) and the second estimate in (156).

For later purposes, we conclude the section with the following remark.

Remark 39. The condition $5 \alpha+2 \beta>6$ in (H1) required in Proposition 29 is the strongest among the conditions for the pair $(\alpha, \beta)$ required in Corollary 14 and the other results of this section. Indeed,

$$
\begin{aligned}
5 \alpha+2 \beta>6 & \Longrightarrow 3 \alpha+2 \beta>6-2 \alpha \geq 4 \\
& \Longrightarrow 3 \alpha+\beta>4-\beta \geq 3 \\
& \Longrightarrow 2 \alpha+\beta>3-\alpha \geq 2 \\
& \Longrightarrow \alpha+\beta>2-\alpha \geq 1
\end{aligned}
$$

Hence, if $5 \alpha+2 \beta>6$, then Corollary 14 and all the results from Lemma 22 to Corollary 38 are applicable. Next we will make large usage of this fact, but we warn the reader that, for brevity and regarding it as acquired, we will not mention it anymore.

\section{Application to Maximal Time Regularity}

The results of the previous sections are here applied to correct, refine, and extend the results in [20] concerning the maximal time regularity of the solutions to a class of degenerate abstract evolution equations. Let $\left(X,\|\cdot\|_{X}\right)$ and $\left(Z,\|\cdot\|_{Z}\right)$ be two complex Banach spaces, and consider the following degenerate first-order integrodifferential Cauchy problem for $v: I_{T} \rightarrow X$, where $I_{T}=[0, T], T>0$, and $n_{1}, n_{2} \in \mathbf{N}:$

$$
\begin{gathered}
D_{t}(M v(t))=\left[\lambda_{0} M+L\right] v(t)+\sum_{i_{1}=1}^{n_{1}} \mathscr{K}\left(k_{i_{1}}, L_{i_{1}} v\right)(t) \\
+\sum_{i_{2}=1}^{n_{2}} h_{i_{2}}(t) y_{i_{2}}+f(t), \quad t \in I_{T}, \\
M v(0)=M v_{0} .
\end{gathered}
$$

Here $\mathscr{K}$ is the convolution operator (104) in which $\left(X_{1}, X_{2}, X_{3}\right)=(Z, X, X)$, whereas $M, L$, and $L_{i_{1}}, i_{1}=$ $1, \ldots, n_{1}$, are closed single-valued linear operators from $X$ to itself, whose domains fulfill the relation $\mathscr{D}(L) \subseteq$ $\bigcap_{i_{1}=1}^{n_{1}}\left[\mathscr{D}(M) \cap \mathscr{D}\left(L_{i_{1}}\right)\right]$. Further, we assume that

$L$ admits a continuous inverse operator $L^{-1} \in \mathscr{L}(X)$,

$$
\text { i.e., } 0 \in \rho(L) \text {, }
$$

whereas we allow $M$ to have no bounded inverse. Hence, in general, $A:=L M^{-1}$ is only the $\mathrm{m}$. l. operator defined by

$$
\begin{aligned}
\mathscr{D}(A)= & \left\{x \in \mathscr{D}\left(M^{-1}\right): L\left(M^{-1} x\right) \neq \emptyset\right\} \\
= & \left\{x \in \mathscr{R}(M): M^{-1} x \cap \mathscr{D}(L) \neq \emptyset\right\} \\
= & \{x \in \mathscr{R}(M): \\
& \text { there exists } \left.y \in \mathscr{D}(L) \text { such that } y \in M^{-1} x\right\} \\
= & \{x \in \mathscr{R}(M): x=M y \text { for some } y \in \mathscr{D}(L)\} \\
= & M(\mathscr{D}(L)), \\
A x= & \bigcup_{y \in M^{-1} x \cap \mathscr{D}(L)} L y \quad x \in \mathscr{D}(A) . \\
= & \{L y: y \in \mathscr{D}(L) \text { such that } x=M y\},
\end{aligned}
$$

Therefore, problem (160) can not be reduced, via the change of unknown $u=M v$, to an integrodifferential problem related to single-valued linear operators. On the contrary, due to (161) and the closed graph theorem, $M L^{-1}, L_{i_{1}} L^{-1} \in$ $\mathscr{L}(X), i_{1}=1, \ldots, n_{1}$. As far as the data vector $\left(\lambda_{0}, v_{0}, k_{1}, \ldots\right.$, $\left.k_{n_{1}}, h_{1}, \ldots, h_{n_{2}}, y_{1}, \ldots, y_{n_{2}}, f\right)$ is concerned, at the moment, we only assume $\lambda_{0} \in \mathbf{C}, v_{0} \in \mathscr{D}(M), k_{i_{1}}: I_{T} \rightarrow Z, h_{i_{2}}$ : $I_{T} \rightarrow \mathrm{C}, y_{i_{2}} \in X, i_{l}=1, \ldots, n_{l}, l=1,2$, and $f: I_{T} \rightarrow X$, in order that (160) makes sense in $X$. This minimal assumptions will be refined later. In general, only strict solutions $v$ to (160) shall be investigated, where (cf. $[22,23]$ ) by a strict solution $v$ to $(160)$ we mean that, $\mathscr{D}(L)$ being endowed with the graph norm $\|\cdot\|_{\mathscr{D}(L)}=\|\cdot\|_{X}+\|L \cdot\|_{X}, v \in C\left(I_{T} ; \mathscr{D}(L)\right)$, $M v \in C^{1}\left(I_{T} ; X\right)$, and (160) holds. Clearly, if $M^{-1}$ is really a m. l. operator, then $M v(0)=M v_{0}$ does not necessarily mean $v(0)=v_{0}$, but only $v(0)-v_{0} \in M^{-1} 0$. As we will see below, if $v_{0} \in \mathscr{D}(L)$ and the data $k_{i_{1}}, h_{i_{2}}, y_{i_{2}}$ and $f, i_{l}=1, \ldots, n_{l}$, $l=1,2$, satisfy suitable assumptions, then for a strict solution $v$ to (160) it just holds $v(0)=v_{0}$. Throughout the section, $Y_{\psi}^{q}$, $\psi \in(0,1), q \in[1, \infty]$, will always denote one between the spaces $(X, \mathscr{D}(A))_{\psi, q}$ and $X_{A}^{\psi, q}, A$ being defined by (162). That is, $Y_{\psi}^{q} \in\left\{(X, \mathscr{D}(A))_{\psi, q}, X_{A}^{\psi, q}\right\}$. To avoid confusion, if more than a single $Y_{\psi}^{q}$ is involved in some statement, that is, if we write $x_{j} \in Y_{\psi_{j}}^{q}, j=1, \ldots, n, n \in \mathbf{N}$, then it is understood that the same choice has been made for all the $Y_{\psi_{j}}^{q}$ in the sense that $Y_{\psi_{j}}^{q}=(X, \mathscr{D}(A))_{\psi_{j}, q}$ or $Y_{\psi_{j}}^{q}=X_{A}^{\psi_{j}, q}$ for every $j=1, \ldots, n$.

According to [2, Section 1.6], we recall that the $M$-modified resolvent set $\rho_{M}(L)$ of $L$ is defined to be the set $\{z \in \mathbf{C}$ : $\left.(z M-L)^{-1} \in \mathscr{L}(X)\right\}$. The bounded operator $(z M-L)^{-1}$ is called the $M$ modified resolvent of $L$. It is easy to prove that $\rho_{M}(L) \subseteq \rho(A)$ and that $M(z M-L)^{-1}=(z I-A)^{-1}, z \in$ $\rho_{M}(L)$ (cf. [2, Theorem 1.14]). With the notion of $M$-modified resolvent of $L$ at hand, we assume that

(H2) $\rho_{M}(L)$ contains a region $\Sigma_{\alpha}=\{z \in \mathbf{C}: \mathfrak{R e} z \geq$ $\left.-c(|\Im \mathrm{m} z|+1)^{\alpha}, \mathfrak{I m} z \in \mathbf{R}\right\}, \alpha \in(0,1], c>0$, and 
for some exponent $\beta \in(0, \alpha]$ and constant $C>0$ the estimate $\left\|M(\lambda M-L)^{-1}\right\|_{\mathscr{L}(X)} \leq C(|\lambda|+1)^{-\beta}$ holds for every $\lambda \in \Sigma_{\alpha}$.

Before we proceed with our analysis we remark that, due to the wide range of choices for the data vector, problem (160) contains many subcases at its interior. So, in spite of the case when at least one between the $k_{i}$ 's is different from zero and problem (160) is really an integrodifferential one, the choice $k_{i_{1}}=0, i_{1}=1, \ldots, n_{1}$, yields to consider also various nonintegrodifferential degenerate problems. For instance, those corresponding to $\lambda_{0}=k_{i_{1}}=h_{i_{2}}=0$ and $\lambda_{0}=k_{i_{1}}=$ $f=0, i_{l}=1, \ldots, n_{l}, l=1,2$, respectively:

$$
\begin{gathered}
D_{t}(M v(t))=L v(t)+f(t), \quad t \in I_{T}, \\
M v(0)=M v_{0}, \\
D_{t}(M v(t))=L v(t)+\sum_{i_{2}=1}^{n_{2}} h_{i_{2}}(t) y_{i_{2}}, \quad t \in I_{T}, \\
M v(0)=M v_{0} .
\end{gathered}
$$

Although (164) differs from (163) only in the fact that $f$ is replaced with $\sum_{i_{2}=1}^{n_{2}} h_{i_{2}}(t) y_{i_{2}}$; nevertheless a very different result is achieved when the $y_{i_{2}}$ 's are assumed to belong to $Y_{\gamma_{i_{2}}}^{r}$, at least for opportunely chosen $\gamma_{i_{2}} \in(0,1), i_{2}=1, \ldots, n_{2}$. As we will see (cf. Remark 51 and Theorem 56), in this situation the loss of time regularity for the pair $\left(L v, D_{t} M v\right)$ with respect to that of $f$, typical of the case $\beta<1$ in (H2) (see [21, Theorem 9], [2, Theorem 3.26], and [22, Theorem 7.2]), can be restored in order that $\left(L v, D_{t} M v\right)$ possesses the maximal time regularity which is the minimal between the time regularities of the $h_{i_{2}}$ 's. The same phenomenon is carried over into the integrodifferential case for the following problems, corresponding to $\lambda_{0}=h_{i_{2}}=0, i_{2}=1, \ldots, n_{2}$, and $\lambda_{0}=f=0$ :

$$
\begin{gathered}
D_{t}(M v(t))=L v(t)+\sum_{i_{1}=1}^{n_{1}} \mathscr{K}\left(k_{i_{1}}, L_{i_{1}} v\right)(t)+f(t), \\
M v(0)=M v_{0}, \\
D_{t}(M v(t))=L v(t)+\sum_{i_{1}=1}^{n_{1}} \mathscr{K}\left(k_{i_{1}}, L_{i_{1}} v\right)(t)+\sum_{i_{2}=1}^{n_{2}} h_{i_{2}}(t) y_{i_{2}}, \\
M v(0)=M v_{0},
\end{gathered}
$$

$t \in I_{T}$. When $\beta<1$, the loss of time regularity for the pair $\left(L v, D_{t} M v\right)$ with respect to that of the vector $\left(k_{1}, \ldots, k_{n_{1}}, f\right)$ in problem (165) (cf. [22, Theorem 7.1] and [23, Theorem 2.1] for $n_{1}=1$ ) can be restored in problem (166) assuming that $y_{i_{2}} \in Y_{\gamma_{i_{2}}}^{r}, i_{2}=1, \ldots, n_{2}$. In this context (cf. Remark 51 and Theorem 53) the pair ( $\left.L v, D_{t} M v\right)$ has the maximal time regularity which is the minimal between the time regularities of the $k_{i_{1}}$ 's and $h_{i_{2}}$ 's.

We stress that, if $\beta=1$, then no loss of time regularity is observed and all the quoted results agree with the wellknown theory of maximal regularity in spaces of continuous functions for the nondegenerate version of (160), corresponding to the case when $M=I$ and $L$ generates an analytic semigroup. Hence, roughly speaking, one can verify the consistency of any result on problem (160) with condition (H2) simply by letting $\beta=1$ on its statement, and then checking if it is compatible with those for the nondegenerate case. To this purpose, we recall that the question of maximal regularity for the nondegenerate (possibly nonautonomous) version of (160) has been deeply investigated by several authors. See, for instance, $[4,6-8,10,32]$ for problem (165) with $\left(M, \beta, n_{1}\right)=(I, 1,1)$ and $[9,11]$ for problem (163) with $(M, \beta)=(I, 1)$.

Finally, assumption (161) excludes the case of $L=0$ in (160), so that our results cannot be compared with those in [5, $33,34]$. There, assuming that the bilinear bounded operator $\mathscr{P}$ underlying the definition of $\mathscr{K}$ is the scalar multiplication in $X$, the problem

$$
D_{t} v(t)=\mathscr{K}\left(k_{1}, L_{1} v\right)(t)+f(t), \quad t \in I_{T}, \quad v(0)=v_{0}
$$

is treated under the following assumptions: (i) $L_{1}$ is a closed densely defined linear operator generating an analytic semigroup; (ii) $k_{1}:[0, \infty) \rightarrow \mathbf{R}$ is absolutely Laplace transformable. Observe that, if $\left(k_{1}, f\right)=(1,0)$, then problem (167) reduces to the abstact wave equation $D_{t}^{2} v(t)=L_{1} v(t)$, $D_{t} v(0)=0, v(0)=v_{0}$, whereas when $M=I$ and $\lambda_{0}=k_{i_{1}}=$ $h_{i_{2}}=f=0, i_{l}=1, \ldots, n_{l}, l=1,2$, problem (160) reduces to the abstract heat equation $D_{t} v(t)=L v(t), v(0)=v_{0}$. In other words, whereas $[5,33,34]$ are concerned with the hyperbolic case, here we are concerned with the parabolic one.

Let us now come back to problem (160). Of course, assumption (H2) implies that the operator $A$ defined by (162) satisfies (H1), so that it generates a semigroup $\left\{\mathrm{e}^{t A}\right\}_{t \geq 0}$ defined by $\mathrm{e}^{0 A}=I$ and (9) and satisfying (14). Assuming that $v_{0} \in$ $\mathscr{D}(L)$, we let

$$
w=L\left(v-v_{0}\right) \Longleftrightarrow v=L^{-1} w+v_{0} .
$$

Then, by setting

$$
\begin{aligned}
F_{w}(t)= & \lambda_{0} A^{-1} w(t) \\
& +\sum_{i_{1}=1}^{n_{1}}\left[\mathscr{K}\left(k_{i_{1}}, S_{i_{1}} w\right)(t)+\mathscr{K}\left(k_{i_{1}}, L_{i_{1}} v_{0}\right)(t)\right] \\
& +\sum_{i_{2}=1}^{n_{2}} h_{i_{2}}(t) y_{i_{2}}+v_{1}+f(t), \quad t \in I_{T}
\end{aligned}
$$

where $A^{-1}=M L^{-1} \in \mathscr{L}(X), S_{i_{1}}=L_{i_{1}} L^{-1} \in \mathscr{L}(X), i_{1}=$ $1, \ldots, n_{1}$, and $v_{1}=\left(\lambda_{0} M+L\right) v_{0}$, we see that $v$ is a strict solution to (160) if and only if $w$ satisfies (indeed, if $v \in$ $C\left(I_{T} ; \mathscr{D}(L)\right)$, then $\|w(t)-w(s)\|_{X}=\|L[v(t)-v(s)]\|_{X} \leq$ $\|v(t)-v(s)\|_{\mathscr{D}(L)} \rightarrow 0$ as $s \rightarrow t, t, s \in I_{T}$, that is, $w \in C$ $\left(I_{T} ; X\right)$. Conversely, if $w \in C\left(I_{T} ; X\right)$, then $v=L^{-1} w+v_{0} \in$ $\mathscr{D}(L)$ and $\|v(t)-v(s)\|_{\mathscr{D}(L)} \leq\left(\left\|L^{-1}\right\|_{\mathscr{L}(X)}+1\right) \| w(t)-$ $w(s) \|_{X} \rightarrow 0$ as $s \rightarrow t, t, s \in I_{T}$, that is, $v \in C\left(I_{T} ; \mathscr{D}(L)\right)$. Finally, since $M v=A^{-1} w+M v_{0}$, we have $M v \in C^{1}\left(I_{T} ; X\right)$ 
if and only if $\left.A^{-1} w \in C^{1}\left(I_{T} ; X\right)\right) w \in C\left(I_{T} ; X\right), A^{-1} w \in$ $C^{1}\left(I_{T} ; X\right)$, and solves to the following problem:

$$
\begin{gathered}
D_{t}\left(A^{-1} w(t)\right)=w(t)+F_{w}(t) \in A\left(A^{-1} w(t)\right)+F_{w}(t), \\
t \in I_{T}, \\
\left.A^{-1} w(0)=0 \quad \text { (i.e., } w(0) \in A 0\right) .
\end{gathered}
$$

Now let $2 \alpha+\beta>2$, and assume that $k_{i_{1}} \in C^{\eta_{i_{1}}}\left(I_{T} ; Z\right), h_{i_{2}} \in$ $C^{\sigma_{i_{2}}}\left(I_{T} ; \mathbf{C}\right)$, and $f \in C^{\mu}\left(I_{T} ; X\right)$, where $\eta_{i_{1}}, \sigma_{i_{2}}, \mu \in(2-\alpha-$ $\beta / \alpha, 1), i_{l}=1, \ldots, n_{l}, l=1,2$. Then, if $w \in C\left(I_{T} ; X\right)$ is a solution to (170) such that $A^{-1} w \in C^{1}\left(I_{T} ; X\right)$, the function $F_{w}$ satisfies

$$
\begin{gathered}
F_{w} \in C^{\delta}\left(I_{T} ; X\right), \\
\delta=\min _{i_{k}=1, \ldots, n_{k}, k=1,2}\left\{\eta_{i_{1}}, \sigma_{i_{2}}, \mu\right\} \in\left(\frac{2-\alpha-\beta}{\alpha}, 1\right) .
\end{gathered}
$$

Indeed, $\delta$ being the smallest Hölder exponent, for every $i_{l}=$ $1, \ldots, n_{l}, l=1,2$, we have $A^{-1} w, h_{i_{2}} y_{i_{2}}, f \in C^{\delta}\left(I_{T} ; X\right)$ and $\mathscr{K}\left(k_{i_{1}}, S_{i_{1}} w\right), \mathscr{K}\left(k_{i_{1}}, L_{i_{1}} v_{0}\right) \in C_{0}^{\eta_{i_{1}}}\left(I_{T} ; X\right) \hookrightarrow C_{0}^{\delta}\left(I_{T} ; X\right)$ (cf. Lemma 27 for the case $\left(\delta_{3_{1}}, \delta_{3_{2}}, X_{1}, X_{2}\right)=\left(\eta_{i_{1}}, 0, Z, X\right)$ with the pair $\left(g_{3_{1}}, g_{3_{2}}\right)$ being replaced by (in fact, since $S_{i_{1}}=$ $L_{i_{1}} L^{-1} \in \mathscr{L}(X), i_{1}=1, \ldots, n_{1}$, if $w \in C\left(I_{T} ; X\right)$, then $S_{i_{1}} w \in$ $C\left(I_{T} ; X\right)$, whereas the constant functions $\kappa_{i_{1}}(t)=L_{i_{1}} v_{0}, t \in$ $I_{T}, i_{1}=1, \ldots, n_{1}$, obviously belong to $\left.C\left(I_{T} ; X\right)\right)\left(k_{i_{1}}, S_{i_{1}} w\right)$ and $\left(k_{i_{1}}, L_{i_{1}} v_{0}\right)$, resp.). Consequently (cf. [2, Theorem 3.7 and Remark p. 54] with $u_{0}=0$ ), the solution $A^{-1} w$ to the multivalued evolution problem $D_{t}\left(A^{-1} w\right) \in A\left(A^{-1} w\right)+F_{w}$, $A^{-1} w(0)=0$ is necessarily of the form

$$
A^{-1} w(t)=\left[Q_{1} F_{w}\right](t), \quad t \in I_{T},
$$

with $Q_{1}$ being the operator defined by (105). Further (cf. [2, Remark p. 55] with $u_{0}=0$, and where $A^{\circ} \mathrm{e}^{t A}$ stands for $\left.D_{t} \mathrm{e}^{t A}=-\left[(-A)^{1}\right]^{\circ} \mathrm{e}^{t A}\right)$ the derivative of $A^{-1} w$ is given by

$$
D_{t}\left(A^{-1} w(t)\right)=\mathrm{e}^{t A} F_{w}(t)-\left[Q_{2} F_{w}\right](t), \quad t \in I_{T} \backslash\{0\},
$$

with $Q_{2}$ being the operator in (106). Notice that $Q_{2} F_{w}$ is well defined by virtue of (127) with $g=F_{w}$. Now let $y_{i_{2}} \in Y_{\gamma_{2}}^{r}$ and $v_{1}+f(0) \in Y_{\varphi}^{r}$ where $\gamma_{i_{2}}, \varphi \in(1-\beta, 1), i_{2}=1, \ldots$, $n_{2}$, and $r \in[1, \infty]$. Since $A^{-1} w(0)=\mathscr{K}\left(k_{i_{1}}, S_{i_{1}} w\right)(0)=$ $\mathscr{K}\left(k_{i_{1}}, L_{i_{1}} v_{0}\right)(0)=0, i_{1}=1, \ldots, n_{1}$, from (169) it thus follows that $F_{w}(0):=x_{0}$ is independent on $w$ and

$$
\begin{gathered}
x_{0}=\sum_{i_{2}=1}^{n_{2}} h_{i_{2}}(0) y_{i_{2}}+v_{1}+f(0) \in Y_{\gamma}^{r}, \\
\gamma=\min _{i_{2}=1, \ldots, n_{2}}\left\{\gamma_{i_{2}}, \varphi\right\} \in(1-\beta, 1) .
\end{gathered}
$$

Indeed (cf. (20) or (38)), we have $Y_{\gamma_{i_{2}}}^{r} \hookrightarrow Y_{\gamma}^{r}, i_{2}=1, \ldots, n_{2}$, and $Y_{\varphi}^{r} \hookrightarrow Y_{\gamma}^{r}$, the embeddings being equalities for those between the numbers $\gamma_{1}, \ldots, \gamma_{n_{2}}$ and $\varphi$ which are equal to $\gamma$. Then, under these assumptions on the data, formula (173) for $D_{t}\left(A^{-1} w(t)\right)$ can be extended until $t=0$. For, we have $\lim _{t \rightarrow 0^{+}} D_{t}\left(A^{-1} w(t)\right)=x_{0} \in A 0+x_{0}$ and the differential equation in (170) is satisfied even at $t=0$. To see this, we observe that

$$
\begin{array}{r}
\left\|D_{t}\left(A^{-1} w(t)\right)-x_{0}\right\|_{X} \leq I_{1}(t)+I_{2, w}(t)+I_{3, w}(t), \\
t \in I_{T} \backslash\{0\},
\end{array}
$$

where $I_{1}(t)=\left\|\left(\mathrm{e}^{t A}-I\right) x_{0}\right\|_{X}, I_{2, w}(t)=\left\|\mathrm{e}^{t A}\left[F_{w}(t)-x_{0}\right]\right\|_{X}$, and $I_{3, w}(t)=\left\|\left[Q_{2} F_{w}\right](t)\right\|_{X}$. First, from Proposition 6 we get $\lim _{t \rightarrow 0^{+}} I_{1}(t)=0$. On the other side, using $F_{w} \in C^{\delta}\left(I_{T} ; X\right)$, $\delta \in((2-\alpha-\beta) / \alpha, 1) \subseteq((1-\beta) / \alpha, 1)$, we obtain

$$
I_{2, w}(t) \leq \widetilde{c}_{\alpha, \beta, 0}\left\|F_{w}\right\|_{\delta, 0, t ; X} t^{(\alpha \delta+\beta-1) / \alpha}, \quad t \in I_{T} \backslash\{0\},
$$

so that $\lim _{t \rightarrow 0^{+}} I_{2, w}(t)=0$. Finally, (127) with $g=F_{w}$ yields $\lim _{t \rightarrow 0^{+}} I_{3, w}(t)=0$, too. Formula (173) thus holds at $t=0$ with $D_{t}\left(A^{-1} w(0)\right)=\lim _{t \rightarrow 0^{+}} D_{t}\left(A^{-1} w(t)\right)=x_{0}$.

Remark 40. In [2, Remark p. 55], formula (173) was extended until $t=0$ only under the more restrictive assumption $x_{0} \in$ $X_{A}^{\gamma, \infty}, \gamma \in(1-\beta, 1)$. Indeed [24, Proposition 5.2] was not available at the time of [2] and only the strong continuity of $\left\{\mathrm{e}^{t A}\right\}_{t \geq 0}$ in the $X$-norm on the spaces $X_{A}^{\gamma, \infty}, \gamma \in(1-\beta, 1)$, was known (cf. [2, Theorem 3.3]). Notice that in the case of problem (163) the element $x_{0}$ reduces to $L v_{0}+f(0)$, so that in the nondegenerate case $(M, \beta)=(I, 1)$ we get back the classical assumption $L v_{0}+f(0) \in(X, \mathscr{D}(L))_{\gamma, r}, \gamma \in(0,1)$, $r \in[1, \infty]$ (see, for instance, [9, Theorem 4.3.1(iii)] and [11, Theorem 4.5]).

Since (170) implies that $w(t)=D_{t}\left(A^{-1} w(t)\right)-F_{w}(t)$, from (173) we thus find that

$$
\begin{array}{r}
w(t)=\left[Q_{7} x_{0}\right](t)+\left(\mathrm{e}^{t A}-I\right)\left[F_{w}(t)-x_{0}\right]-\left[Q_{2} F_{w}\right](t), \\
t \in I_{T},
\end{array}
$$

where, according to the notation in Corollary 38, we have set $\left[Q_{7} x_{0}\right](t)=\left(\mathrm{e}^{t A}-I\right) x_{0}$. In particular, $w(0)=0$. We conclude that, under the previous assumptions on the pair $(\alpha, \beta)$ and on the data vector $\left(k_{1}, \ldots, k_{n_{1}}, h_{1}, \ldots, h_{n_{2}}, y_{1}, \ldots, y_{n_{2}}, f, v_{1}\right)$, if $w \in C\left(I_{T} ; X\right)$ solves $(170)$, then necessarily $w \in C_{0}\left(I_{T} ; X\right)$. As a consequence (cf. (168)), the strict solution $v$ to (160) satisfies the initial condition just in the sense $v(0)=v_{0}$.

Introduce the functions $\tilde{f}: I_{T} \rightarrow X$ and $\widetilde{h}_{i_{2}}: I_{T} \rightarrow Y_{\gamma_{i_{2}}}^{r}$, $i_{2}=1, \ldots, n_{2}$, defined by

$$
\begin{array}{r}
\tilde{f}(t)=f(t)-f(0), \quad \widetilde{h}_{i_{2}}(t)=\left[h_{i_{2}}(t)-h_{i_{2}}(0)\right] y_{i_{2}}, \\
t
\end{array}
$$

Then, replacing $F_{w}$ with the right-hand side of (169), using (174), and recalling the definitions of the operators $Q_{j}$, 
$j=2, \ldots, 6$, in (106)-(110), from (177) we deduce that $w \in C_{0}$ $\left(I_{T} ; X\right)$ solves the fixed-point equation

$$
w=w_{0}+w_{1}+R w
$$

the functions $w_{l}, l=0,1$, and the operator $R w$ being defined by

$$
\begin{aligned}
w_{0}:=Q_{7} x_{0}+\sum_{i_{1}=1}^{n_{1}} Q_{6}\left(k_{i_{1}}, L_{i_{1}} v_{0}\right)+\sum_{i_{2}=1}^{n_{2}} Q_{5} \tilde{h}_{i_{2}}+Q_{5} \tilde{f} \\
w_{1}:=-\sum_{i_{1}=1}^{n_{1}} Q_{3}\left(k_{i_{1}}, L_{i_{1}} v_{0}\right)-\sum_{i_{2}=1}^{n_{2}} Q_{4}\left(h_{i_{2}}, y_{i_{2}}\right)-Q_{2} f \\
R w:=\lambda_{0}\left[Q_{5}\left(A^{-1} w\right)-Q_{2}\left(A^{-1} w\right)\right] \\
\quad+\sum_{i_{1}=1}^{n_{1}}\left[Q_{6}\left(k_{i_{1}}, S_{i_{1}} w\right)-Q_{3}\left(k_{i_{1}}, S_{i_{1}} w\right)\right] .
\end{aligned}
$$

Conversely, let $w \in C_{0}\left(I_{T} ; X\right)$ be a solution to the fixedpoint equation (179), and assume that the pair $(\alpha, \beta)$ and the data vector $\left(k_{1}, \ldots, k_{n_{1}}, h_{1}, \ldots, h_{n_{2}}, y_{1}, \ldots, y_{n_{2}}, f, v_{1}\right)$ satisfy the assumptions below (170) and (173). Then, as before, $\mathscr{K}\left(k_{i_{1}}, S_{i_{1}} w\right), \mathscr{K}\left(k_{i_{1}}, L_{i_{1}} v_{0}\right) \in C_{0}^{\delta}\left(I_{T} ; X\right)$ and $h_{i_{2}} y_{i_{2}}, f \in$ $C^{\delta}\left(I_{T} ; X\right), i_{l}=1, \ldots, n_{l}, l=1,2, \delta \in((2-\alpha-\beta) / \alpha, 1)$ being as in (171). We apply $A^{-1} \in \mathscr{L}(X)$ to both sides of (179), and we show that $A^{-1} w$ satisfies (172) with $F_{w} \in C\left(I_{T} ; X\right)$ as in (169), so that $A^{-1} w$ is a solution to problem (170). To this purpose, we take into account Corollaries 14 and 26. Let $t \in I_{T}$. First (cf. Remark 34 and recall that $Q_{6}(\cdot, \cdot)=Q_{5} \mathscr{K}(\cdot, \cdot)$ ), using $(86)$, (174), and (178), we get

$$
\begin{aligned}
& A^{-1} w_{0}(t) \int_{0}^{t} \mathrm{e}^{(t-s) A}\left[x_{0}+\sum_{i_{1}=1}^{n_{1}} \mathscr{K}\left(k_{i_{1}}, L_{i_{1}} v_{0}\right)(t)\right. \\
&\left.+\sum_{i_{2}=1}^{n_{2}} \tilde{h}_{i_{2}}(t)+\tilde{f}(t)\right] \mathrm{d} s \\
&=\int_{0}^{t} \mathrm{e}^{(t-s) A}\left[\begin{array}{l}
v_{1}+\sum_{i_{1}=1}^{n_{1}} \mathscr{K}\left(k_{i_{1}}, L_{i_{1}} v_{0}\right)(t) \\
\left.+\sum_{i_{2}=1}^{n_{2}} h_{i_{2}}(t) y_{i_{2}}+f(t)\right] \mathrm{d} s .
\end{array}\right.
\end{aligned}
$$
obtain

Instead, due to the definition of $Q_{3}$ and $Q_{4}$, using (125) we

$$
\begin{aligned}
& A^{-1} w_{1}(t) \\
& =\int_{0}^{t} \mathrm{e}^{(t-s) A}\left\{\sum_{i_{1}=1}^{n_{1}}\left[\mathscr{K}\left(k_{i_{1}}, L_{i_{1}} v_{0}\right)(s)-\mathscr{K}\left(k_{i_{1}}, L_{i_{1}} v_{0}\right)(t)\right]\right. \\
& \left.+\sum_{i_{2}=1}^{n_{2}}\left[h_{i_{2}}(s)-h_{i_{2}}(t)\right] y_{i_{2}}+f(s)-f(t)\right\} \mathrm{d} s .
\end{aligned}
$$

Therefore, from (183), (184), and the definition (105) of $Q_{1}$ it follows that

$$
\begin{aligned}
A^{-1} & {\left[w_{0}+w_{1}\right](t) } \\
& =\left[Q_{1}\left(v_{1}+\sum_{i_{1}=1}^{n_{1}} \mathscr{K}\left(k_{i_{1}}, L_{i_{1}} v_{0}\right)+\sum_{i_{2}=1}^{n_{2}} h_{i_{2}} y_{i_{2}}+f\right)\right](t),
\end{aligned}
$$

the left-hand side being well-defined due to Remark 23. As far as $A^{-1}[R w](t)$ is concerned, we first observe that, $w$ being in $C\left(I_{T} ; X\right)$, from formula (126) and Remark 34 it follows that $\left[Q_{2}\left(A^{-1} w\right)\right](t)$ and $\left[Q_{5}\left(A^{-1} w\right)\right](t)$ are both well defined and equal to $-\int_{0}^{t} \mathrm{e}^{(t-s) A}[w(s)-w(t)] \mathrm{d} s$ and $\int_{0}^{t} \mathrm{e}^{(t-s) A} w(t) \mathrm{d} s$, respectively. Consequently

$$
\begin{aligned}
{\left[Q_{5}\left(A^{-1} w\right)-Q_{2}\left(A^{-1} w\right)\right](t) } \\
\quad=\int_{0}^{t} \mathrm{e}^{(t-s) A} w(s) \mathrm{d} s=\left[Q_{1} w\right](t) .
\end{aligned}
$$

Hence, commuting $A^{-1} \in \mathscr{L}(X)$ with both the integral sign and the semigroup, one has

$$
A^{-1}\left[Q_{5}\left(A^{-1} w\right)-Q_{2}\left(A^{-1} w\right)\right](t)=\left[Q_{1}\left(A^{-1} w\right)\right](t) .
$$

Similarly, since Remark 34 and formula (125) yield

$$
\begin{aligned}
& A^{-1}\left[Q_{6}\left(k_{i_{1}}, S_{i_{1}} w\right)\right](t)=\int_{0}^{t} \mathrm{e}^{(t-s) A} \mathscr{K}\left(k_{i_{1}}, S_{i_{1}} w\right)(t) \mathrm{d} s, \\
& A^{-1}\left[Q_{3}\left(k_{i_{1}}, S_{i_{1}} w\right)\right](t) \\
& =-\int_{0}^{t} \mathrm{e}^{(t-s) A}\left[\mathscr{K}\left(k_{i_{1}}, S_{i_{1}} w\right)(s)-\mathscr{K}\left(k_{i_{1}}, S_{i_{1}} w\right)(t)\right] \mathrm{d} s,
\end{aligned}
$$

we find that

$$
\begin{aligned}
A^{-1}\left[Q_{6}\left(k_{i_{1}}, S_{i_{1}} w\right)-Q_{3}\left(k_{i_{1}}, S_{i_{1}} w\right)\right](t) \\
=\left[Q_{1} \mathscr{K}\left(k_{i_{1}}, S_{i_{1}} w\right)\right](t),
\end{aligned}
$$

$i_{1}=1, \ldots, n_{1}$. In conclusion, from (187) and (189) it follows that

$$
A^{-1}[R w](t)=\left[Q_{1}\left(\lambda_{0} A^{-1} w+\sum_{i_{1}=1}^{n_{1}} \mathscr{K}\left(k_{i_{1}}, S_{i_{1}} w\right)\right)\right](t) .
$$

Summing up (185) and (190), we finally obtain $A^{-1} w(t)=$ $\left[Q_{1} F_{w}\right](t), F_{w}$ being as in (169). This completes the proof of the equivalence between problem (170) and the fixed point equation (179), provided that the data satisfy the mentioned assumptions.

Remark 41. We can summarize the previous reasonings as follows: problem (160) has been reduced to the fixed-point 
equation (179) for the new unknow $w=L\left(v-v_{0}\right), v_{0} \in \mathscr{D}(L)$. This fixed-point argument is similar to that first successfully applied in $[4,7,8,32]$ to problem $(165)$ with $\left(M, \beta, n_{1}\right)=$ $(I, 1,1)$ and then generalized in $[23]$ to the degenerate case. A different approach has been followed in $[6,10]$ for the nondegenerate case and in [22] for the degenerate one. There, assuming that $k_{1}$ is absolutely Laplace transformable (cf. $[6,22])$ or of bounded variation (cf. [10]), problem (165) with $n_{1}=1$ is solved by constructing its relative resolvent operator. We quote also [35] where the method of constructing the fundamental solution for the equation without the integral term is applied to a class of concrete degenerate integrodifferential equations.

From now on, for $5 \alpha+2 \beta>6, \beta \in(0, \alpha], \alpha \in(0,1]$, and $\nu \in((3-2 \alpha-\beta) / \alpha, 1), I_{\alpha, \beta, \nu} \subseteq((3-2 \alpha-\beta) / \alpha, 1 / 2) \subseteq(0,1 / 2)$ will denote the interval defined by

$$
\begin{aligned}
& I_{\alpha, \beta, \nu} \\
& = \begin{cases}\left(\frac{3-2 \alpha-\beta}{\alpha}, v\right], & \text { if } \nu \in\left(\frac{3-2 \alpha-\beta}{\alpha}, \frac{1}{2}\right), \\
\left(\frac{3-2 \alpha-\beta}{\alpha}, \frac{1}{2}\right), & \text { if } \nu \in\left[\frac{1}{2}, 1\right) .\end{cases}
\end{aligned}
$$

Clearly, if $\nu, \rho \in((3-2 \alpha-\beta) / \alpha, 1), \nu \leq \rho$, then $I_{\alpha, \beta, \nu} \subseteq I_{\alpha, \beta, \rho}$.

Lemma 42. Assume (161), and let $5 \alpha+2 \beta>6$ in (H2). Assume that $k_{i_{1}} \in C^{\eta_{i_{1}}}\left(I_{T} ; Z\right), \eta_{i_{1}} \in((3-2 \alpha-\beta) / \alpha, 1), i_{1}=1, \ldots, n_{1}$, and let $\eta=\min _{i_{1}=1, \ldots, n_{1}} \eta_{i_{1}}$. Then, for every fixed $\delta \in I_{\alpha, \beta, \eta}$, the operator $R$ defined by (182) maps continuously $C^{\delta}\left(I_{T} ; X\right)$ into $C_{0}^{\delta}\left(I_{T} ; X\right)$, and for every $t \in I_{T}$ satisfies the following estimate, where $p \in(1 /(1-2 \delta), \infty)$ :

$$
\|R w\|_{\delta, 0, t ; X} \leq c_{42}(T)\left(\int_{0}^{t}\|w\|_{\delta, 0, s ; X}^{p} \mathrm{~d} s\right)^{1 / p}, \quad w \in C^{\delta}\left(I_{T} ; X\right)
$$

Here $c_{42}(T)$ is a positive constant depending only on $T, \lambda_{0}, \alpha$, $\beta, \eta_{i_{1}}, \delta, p,\left\|k_{i_{1}}\right\|_{\eta_{i_{1}}, 0, T ; Z}$ and $\left\|S_{i_{1}}\right\|_{\mathscr{L}(X)}, i_{1}=1, \ldots, n_{1}$.

Proof. Let $k_{i_{1}} \in C^{\eta_{i}}\left(I_{T} ; Z\right), \eta_{i_{1}} \in((3-2 \alpha-\beta) / \alpha, 1), i_{1}=1$, $\ldots, n_{1}$, and let us fix an arbitrary number $\delta \in I_{\alpha, \beta, \eta}$, where $\eta=\min _{i_{1}=1, \ldots, n_{1}} \eta_{i}$. In particular, since $\delta \leq \eta \leq \eta_{i}$, we have $k_{i_{1}} \in C^{\delta}\left(I_{T} ; Z\right)$ with $\left\|k_{i_{1}}\right\|_{\delta, 0, t ; Z} \leq \max \left\{1, t^{\eta_{i_{1}}-\delta}\right\}\left\|k_{i_{1}}\right\|_{\eta_{i_{1}}, 0, t ; Z}$, $i_{1}=1, \ldots, n_{1}$. Now let $w \in C^{\delta}\left(I_{T} ; X\right)$ and $t \in I_{T}$. First, formula (186) being applicable, we rewrite (182) as

$$
R w=\lambda_{0} Q_{1} w+\sum_{i_{1}=1}^{n_{1}}\left[Q_{6}\left(k_{i_{1}}, S_{i_{1}} w\right)-Q_{3}\left(k_{i}, S_{i} w\right)\right]
$$

Now, we notice that $5 \alpha+2 \beta>6$ implies that

$$
\frac{\alpha+\beta-1}{\alpha}=\frac{5 \alpha+2 \beta-3 \alpha-2}{2 \alpha}>\frac{4-3 \alpha}{2 \alpha} \geq \frac{1}{2}
$$

Since $(1-\beta) / \alpha \leq(2-\alpha-\beta) / \alpha \leq(3-2 \alpha-\beta) / \alpha$, from (194) it follows that $\delta \in I_{\alpha, \beta, \eta} \subseteq((3-2 \alpha-\beta) / \alpha, 1 / 2) \subseteq((2-\alpha-$ $\beta) / \alpha, 1 / 2) \varsubsetneqq((1-\beta) / \alpha,(\alpha+\beta-1) / \alpha)$, and, consequently,

$$
\frac{\alpha}{\alpha+\beta-1-\alpha \delta}<\frac{1}{1-2 \delta} \text {. }
$$

We conclude (cf. Remark 39) that Lemma 22 and Propositions 29 and 36 are applicable with $\delta \in I_{\alpha, \beta, \eta}$ and $p \in(1 /(1-$ $2 \delta), \infty)$. Then, using estimates (111), (135), and (150) with the pair $\left(g_{1}, \delta_{1}\right)$ and the quintuplets $\left(g_{l_{1}}, g_{l_{2}}, \delta_{l}, X_{1}, X_{2}\right), l=3,6$, being replaced, respectively, by $(w, \delta)$ and (indeed, since $S_{i_{1}}=$ $L_{i_{1}} L^{-1} \in \mathscr{L}(X)$, if $w \in C^{\delta}\left(I_{T} ; X\right)$, then $S_{i_{1}} w \in C^{\delta}\left(I_{T} ; X\right)$ with $\left.\left\|S_{i_{1}} w\right\|_{\delta, 0, t ; X} \leq\left\|S_{i_{1}}\right\|_{\mathscr{L}(X)}\|w\|_{\delta, 0, t ; X}, i_{1}=1, \ldots, n_{1}\right)\left(k_{i_{1}}, S_{i_{1}} w\right.$, $\delta, Z, X), i_{1}=1, \ldots, n_{1}$, from (193) we finally obtain

$$
\begin{aligned}
\|R w\|_{\delta, 0, t ; X} \leq & \left\|\lambda_{0} Q_{1} w\right\|_{\delta, 0, t ; X} \\
& +\sum_{l=3,6, i_{1}=1, \ldots, n_{1}}\left\|Q_{l}\left(k_{i_{1}}, S_{i_{1}} w\right)\right\|_{\delta, 0, t ; X} \\
\leq & c_{42}(T)\left(\int_{0}^{t}\|w\|_{\delta, 0, s ; X}^{p} \mathrm{~d} s\right)^{1 / p} .
\end{aligned}
$$

Here we have set $c_{42}(T)=\left|\lambda_{0}\right| C_{1}(T)+$ $T^{1-2 \delta-1 / p} \sum_{l=3,6, i_{1}=1, \ldots, n_{1}} C_{l}(T)\left\|k_{i_{1}}\right\|_{\delta, 0, T ; Z}\left\|S_{i_{1}}\right\|_{\mathscr{L}(X)}$, where $C_{l}(T), l=1,3,6$, are the values at $t=T$ of the functions $C_{l}(t)$ in Lemma 22 and Propositions 29 and 36. This completes the proof.

Remark 43. Assume that in Lemma 42 the Hölder exponents $\eta_{i_{1}} \in((3-2 \alpha-\beta) / \alpha, 1)$ are such that $\eta=\min _{i_{1}=1, \ldots, n_{1}} \eta_{i}$ belongs to $((3-2 \alpha-\beta) / \alpha, 1 / 2)$. In this case (cf. (191)), the choice $\delta=\eta$ is admissible, and the meaning of Lemma 42 is that the operator $R$ defined by (182) preserves the minimal of the time regularities of $k_{1}, \ldots, k_{n_{1}}$.

Corollary 44. Let the assumptions of Lemma 42 be satisfied, and let $\eta$ and $R$ be as there. Then, for every fixed $\delta \in I_{\alpha, \beta, \eta}$, the sequence $\left\{R^{n}\right\}_{n=0}^{\infty}\left(R^{0}=I, R^{n}=R R^{n-1}, n \in \mathrm{N}\right)$ satisfies the following estimates, where $w \in C^{\delta}\left(I_{T} ; X\right)$ and $p \in(1 /(1-$ $2 \delta), \infty)$ :

$$
\begin{array}{r}
\left\|R^{n} w\right\|_{\delta, 0, t ; X} \leq\left[c_{42}(T)\right]^{n}\left(\frac{t^{n}}{n !}\right)^{1 / p}\|w\|_{\delta, 0, T ; X}, \\
t \in I_{T}, \quad n \in \mathbf{N} \cup\{0\} .
\end{array}
$$

Proof. Reasoning as in [23, p. 468], we prove (197) by induction. Since for every fixed $\delta \in I_{\alpha, \beta, \eta}$ the operator $R$ maps $C^{\delta}\left(I_{T} ; X\right)$ in $C_{0}^{\delta}\left(I_{T} ; X\right)$, replacing $w$ with $R^{n} w$ in (192) and introducing the sequence of scalar nonnegative nondecreasing functions $\left\{\varphi_{n}\right\}_{n=0}^{\infty}$ defined by $\varphi_{n}(t)=\left\|R^{n} w\right\|_{\delta, 0, t ; X}, t \in I_{T}$, from (192) we obtain

$$
\begin{array}{r}
\varphi_{n+1}(t) \leq c_{42}(T)\left(\int_{0}^{t}\left|\varphi_{n}(s)\right|^{p} \mathrm{~d} s\right)^{1 / p}, \\
t \in I_{T}, \quad n \in \mathbf{N} \cup\{0\} .
\end{array}
$$


Then, applying to (198) an induction argument in which the first step of the induction follows from (192), we immediately deduce the following estimates:

$$
\begin{array}{r}
\varphi_{n}(t) \leq\left[c_{42}(T)\right]^{n}\left(\frac{t^{n}}{n !}\right)^{1 / p}\|w\|_{\delta, 0, T ; X}, \\
t \in I_{T}, \quad n \in \mathbf{N} \cup\{0\} .
\end{array}
$$

The proof is complete.

Lemma 45. Let $5 \alpha+2 \beta>6$ in (H2) and $v_{0} \in \bigcap_{i_{1}=1}^{n_{1}} \mathscr{D}\left(L_{i_{1}}\right)$. Assume that $k_{i_{1}} \in C^{\eta_{i_{1}}}\left(I_{T} ; Z\right), h_{i_{2}} \in C^{\sigma_{i_{2}}}\left(I_{T} ; \mathbf{C}\right)$, and $y_{i_{2}} \in$ $Y_{\gamma_{i_{2}}}^{r}$, where $\eta_{i_{1}}, \sigma_{i_{2}} \in((3-2 \alpha-\beta) / \alpha, 1), \gamma_{i_{2}} \in(3-2 \alpha-$ $\beta, 1), i_{l}=1, \ldots, n_{l}, l=1,2$, and $r \in[1, \infty]$. Let $\tau_{1}=$ $\min _{i_{l}=1, \ldots, n_{l}, l=1,2}\left\{\eta_{i_{1}}, \sigma_{i_{2}}\right\}$. Then, for every fixed $\delta \in I_{\alpha, \beta, \tau_{1}}$, the function $w_{1}$ defined by (181) belongs to $C_{0}^{\delta}\left(I_{T} ; X\right)$, provided that $f \in C^{\mu}\left(I_{T} ; X\right), \mu \in\left[\delta+\mu_{\alpha, \beta}, 1\right), \mu_{\alpha, \beta}=(3-2 \alpha-\beta) / \alpha$.

Proof. Let us fix $\delta \in I_{\alpha, \beta, \tau_{1}}, \tau_{1}=\min _{i_{l}=1, \ldots, n_{l}, l=1,2}\left\{\eta_{i_{1}}, \sigma_{i_{2}}\right\}$. Of course, $k_{i_{1}} \in C^{\delta}\left(I_{T} ; Z\right)$ and $h_{i_{2}} \in C^{\delta}\left(I_{T} ; \mathbf{C}\right), i_{l}=1, \ldots, n_{l}$, $l=1,2$. Then, Proposition 29 and Lemma 30 applied with the quintuplets $\left(g_{3_{1}}, g_{3_{2}}, \delta_{3}, X_{1}, X_{2}\right)$ and the quadruplet $\left(g_{4}, y, \delta_{4}, \gamma\right)$ being replaced, respectively, by (the constant functions $\kappa_{i_{1}}(t)=L_{i_{1}} v_{0}, t \in I_{T}, i=1, \ldots, n_{1}$, being obviously of class $\left.C^{\delta}\left(I_{T} ; X\right)\right)\left(k_{i_{1}}, L_{i_{1}} v_{0}, \delta, Z, X\right)$ and $\left(h_{i_{2}}, y_{i_{2}}, \delta, \gamma_{i_{2}}\right)$, imply that $Q_{3}\left(k_{i_{1}}, L_{i_{1}} v_{0}\right), Q_{4}\left(h_{i_{2}}, y_{i_{2}}\right) \in C_{0}^{\delta}\left(I_{T} ; X\right), i_{l}=$ $1, \ldots, n_{l}, l=1,2$. Now, since $\delta \in I_{\alpha, \beta, \tau_{1}} \subseteq((3-2 \alpha-$ $\beta) / \alpha, 1 / 2) \subseteq(0,1 / 2)$, the number $\delta+\mu_{\alpha, \beta}$ satisfies

$$
\frac{3-2 \alpha-\beta}{\alpha}<\delta \leq \delta+\mu_{\alpha, \beta}<\frac{6-3 \alpha-2 \beta}{2 \alpha}<1,
$$

and assumption $f \in C^{\mu}\left(I_{T} ; X\right), \mu \in\left[\delta+\mu_{\alpha, \beta}, 1\right)$, is meaningful. Lemma 24 with $\left(g_{2}, \delta_{2}\right)=(f, \mu)$ then yields $Q_{2} f \in C_{0}^{\nu_{\alpha, \beta, \mu}}\left(I_{T} ; X\right), \nu_{\alpha, \beta, \mu}=(\alpha \mu+2 \alpha+\beta-3) / \alpha$. Since $v_{\alpha, \beta, \mu} \geq v_{\alpha, \beta, \delta+\mu_{\alpha, \beta}}=\delta$, we get $Q_{2} f \in C_{0}^{\delta}\left(I_{T} ; X\right)$, too. Summing up, we get the assertion.

Before considering the function $w_{0}$ in (180), we introduce the following notation. In the sequel, for $3 \alpha+2 \beta>4, \beta \in$ $(0, \alpha], \alpha \in(0,1]$, and $\nu \in((2-\alpha-\beta) / \alpha, 1), J_{\alpha, \beta, \nu} \subseteq((2-\alpha-$ $\beta) / \alpha, 1 / 2) \subseteq(0,1 / 2)$ will denote the interval

$$
J_{\alpha, \beta, \nu}= \begin{cases}\left(\frac{2-\alpha-\beta}{\alpha}, \nu\right], & \text { if } \nu \in\left(\frac{2-\alpha-\beta}{\alpha}, \frac{1}{2}\right), \\ \left(\frac{2-\alpha-\beta}{\alpha}, \frac{1}{2}\right), & \text { if } \nu \in\left[\frac{1}{2}, 1\right) .\end{cases}
$$

Notice that, since $(2-\alpha-\beta) / \alpha \leq(3-2 \alpha-\beta) / \alpha$, if the stronger condition $5 \alpha+2 \beta>6$ is satisfied, then (191) and (201) yield $I_{\alpha, \beta, \nu} \subseteq J_{\alpha, \beta, \nu}$ for every fixed $\nu \in((3-2 \alpha-\beta) / \alpha, 1)$. The introduction of the intervals $J_{\alpha, \beta, \nu}$ is justified by Lemma 46, which requires a weaker condition on the pair $(\alpha, \beta)$ than the one in Lemmas 42 and 45.

Lemma 46. Let $3 \alpha+2 \beta>4$ in (H2), and let $v_{0} \in \mathscr{D}(L)$. Assume that $k_{i_{1}} \in C^{\eta_{i_{1}}}\left(I_{T} ; X\right), h_{i_{2}} \in C^{\sigma_{i_{2}}}\left(I_{T} ; \mathbf{C}\right), y_{i_{2}} \in Y_{\gamma_{i_{2}}}^{r}$, and $v_{1}+f(0) \in Y_{\varphi}^{r}$, where $\eta_{i_{1}}, \sigma_{i_{2}} \in((2-\alpha-\beta) / \alpha, 1)$, $\gamma_{i_{2}}, \varphi \in(4-2 \alpha-2 \beta, 1), i_{l}=1, \ldots, n_{l}, l=1,2, r \in[1, \infty]$, and $v_{1}=\left(\lambda_{0} M+L\right) v_{0}$. Let $\gamma=\min _{i_{2}=1, \ldots, n_{2}}\left\{\gamma_{i_{2}}, \varphi\right\}$ and $\tau_{0}=$ $\min _{i_{l}=1, \ldots, n_{l}, l=1,2}\left\{\eta_{i_{1}}, \sigma_{i_{2}}, \chi_{\alpha, \beta, \gamma}\right\}$, where $\chi_{\alpha, \beta, \gamma}=(\alpha+\beta+\gamma-2) / \alpha$. Then, for every fixed $\delta \in J_{\alpha, \beta, \tau_{0}}$, the function $w_{0}$ defined by (180) belongs to $C_{0}^{\delta}\left(I_{T} ; X\right)$, provided that $f \in C^{\mu}\left(I_{T} ; X\right), \mu \in$ $\left[\delta+\varrho_{\alpha, \beta}, 1\right), \varrho_{\alpha, \beta}=(2-\alpha-\beta) / \alpha$.

Proof. Observe that (cf. (159)) all the results from Lemma 32 to Corollary 38 will be applicable. First, since $2 \alpha+2 \beta>$ $4-\alpha \geq 3$, the choice $\gamma_{i_{2}}, \varphi \in(4-2 \alpha-2 \beta, 1), i_{2}=1, \ldots, n_{2}$, is meaningful. Moreover, since $\gamma=\min _{i_{2}=1, \ldots, n_{2}}\left\{\gamma_{i_{2}}, \varphi\right\} \in$ $(4-2 \alpha-2 \beta, 1)$, the number $\chi_{\alpha, \beta, \gamma}=(\alpha+\beta+\gamma-$ $2) / \alpha$ satisfies $\chi_{\alpha, \beta, \gamma} \in((2-\alpha-\beta) / \alpha, 1)$. Hence, $\tau_{0}=$ $\min _{i_{l}=1, \ldots, n_{l}, l=1,2}\left\{\eta_{i_{1}}, \sigma_{i_{2}}, \chi_{\alpha, \beta, \gamma}\right\} \in((2-\alpha-\beta) / \alpha, 1)$, too, and $J_{\alpha, \beta, \tau_{0}}$ is well defined. Now, let $\delta \in J_{\alpha, \beta, \tau_{0}}$ be fixed. Due to (20) or (38), the element $x_{0}$ defined by (174) belongs to $Y_{\gamma}^{r}$, whereas the functions $\tilde{h}_{i_{2}}$ defined by (178) are of class $C_{0}^{\delta}\left(I_{T} ; Y_{\gamma_{i_{2}}}^{r}\right) \hookrightarrow$ $C_{0}^{\delta}\left(I_{T} ; Y_{\gamma}^{r}\right)$. Then, since $\gamma \in(4-2 \alpha-2 \beta, 1) \subseteq(2-\alpha-$ $\beta, 1)$, from Lemma 37 and Corollary 38 applied with the pairs $\left(g_{5}, \delta_{5}\right)$ and $\left(x, \delta_{7}\right)$ being replaced by $\left(\widetilde{h}_{i_{2}}, \delta\right)$ and $\left(x_{0}, \delta\right)$, respectively, we deduce that $Q_{5} \widetilde{h}_{i_{2}}, Q_{7} x_{0} \in C_{0}^{\delta}\left(I_{T} ; X\right), i_{2}=$ $1, \ldots, n_{2}$. In addition, since the $k_{i_{1}}$ 's and the constant functions $\kappa_{i_{1}}(t)=L_{i_{1}} v_{0}$ belong to $C^{\delta}\left(I_{T} ; X\right)$, from Proposition 36 applied with $\left(g_{6_{1}}, g_{6_{2}}, X_{1}, X_{2}\right)=\left(k_{i_{1}}, L_{i_{1}} v_{0}, Z, X\right)$, it follows that $Q_{6}\left(k_{i_{1}}, L_{i_{1}} v_{0}\right) \in C_{0}^{\delta}\left(I_{T} ; X\right), i_{1}=1, \ldots, n_{1}$. Finally, since $\delta \in J_{\alpha, \beta, \tau_{0}} \subseteq((2-\alpha-\beta) / \alpha, 1 / 2)$, the number $\delta+\varrho_{\alpha, \beta}$ satisfies

$$
\frac{2-\alpha-\beta}{\alpha}<\delta \leq \delta+\varrho_{\alpha, \beta}<\frac{4-\alpha-2 \beta}{2 \alpha}<1,
$$

and the assumption $f \in C^{\mu}\left(I_{T} ; X\right), \mu \in\left[\delta+\varrho_{\alpha, \beta}, 1\right)$, makes sense. Then, the function $\tilde{f}=f-f(0)$ being of class $C_{0}^{\mu}\left(I_{T} ; X\right)$, Lemma 32 applied with $\left(g_{5}, \delta_{5}\right)=(\widetilde{f}, \mu)$ yields $Q_{5} \tilde{f} \in C_{0}^{\tilde{\alpha}_{\alpha, \beta, \mu}}\left(I_{T} ; X\right), \widetilde{\nu}_{\alpha, \beta, \mu}=(\alpha \mu+\alpha+\beta-2) / \alpha$. Since $\widetilde{\nu}_{\alpha, \beta, \mu} \geq \widetilde{\nu}_{\alpha, \beta, \delta+\varrho_{\alpha, \beta}}=\delta$, we conclude that $Q_{5} \widetilde{f} \in C_{0}^{\delta}\left(I_{T} ; X\right)$, too. Summing up, we get the assertion.

Remark 47. We stress that, if $\beta \in(0,1)$ in (H2), then $0<$ $\rho_{\alpha, \beta} \leq \mu_{\alpha, \beta}$, so that in both Lemmas 45 and 46 we have to assume that $f \in C^{\mu}\left(I_{T} ; X\right)$ with $\mu>\delta$. This is necessary in order to restore the loss of regularity produced by the operators $Q_{2}$ and $Q_{5}$.

We can now prove the main results of the section.

Theorem 48. Assume (161) and $v_{0} \in \mathscr{D}(L)$, and let $5 \alpha+2 \beta>6$ in (H2). Assume that $k_{i_{1}} \in C^{\eta_{i_{1}}}\left(I_{T} ; Z\right), h_{i_{2}} \in C^{\sigma_{i_{2}}}\left(I_{T} ; \mathrm{C}\right), y_{i_{2}} \in$ $Y_{\gamma_{i_{2}}}^{r}$, and $v_{1}+f(0) \in Y_{\varphi}^{r}$, where $\eta_{i_{1}}, \sigma_{i_{2}} \in((3-2 \alpha-\beta) / \alpha, 1)$, $\gamma_{i_{2}}, \varphi \in(5-3 \alpha-2 \beta, 1), i_{l}=1, \ldots, n_{l}, l=1,2, r \in[1, \infty]$, and $v_{1}=\left(\lambda_{0} M+L\right) v_{0}$. Let $\gamma=\min _{i_{2}=1, \ldots, n_{2}}\left\{\gamma_{i_{2}}, \varphi\right\}$ and $\tau=$ $\min _{i_{l}=1, \ldots, n_{l}, l=1,2}\left\{\eta_{i_{1}}, \sigma_{i_{2}}, \chi_{\alpha, \beta, \gamma}\right\}$, where $\chi_{\alpha, \beta, \gamma}=(\alpha+\beta+\gamma-2) / \alpha$. Then, for every fixed $\delta \in I_{\alpha, \beta, \tau}$ problem (160) admits a unique strict solution $v \in C^{\delta}\left(I_{T} ; \mathscr{D}(L)\right)$ satisfying $v(0)=v_{0}$ and such that $L v, D_{t} M v \in C^{\delta}\left(I_{T} ; X\right)$, provided that $f \in C^{\mu}\left(I_{T} ; X\right), \mu \in$ $\left[\delta+\mu_{\alpha, \beta}, 1\right), \mu_{\alpha, \beta}=(3-2 \alpha-\beta) / \alpha$. 
Proof. Of course, due to (159), the assumption $\gamma_{i_{2}}, \varphi \in(5-$ $3 \alpha-2 \beta, 1), i_{2}=1, \ldots, n_{2}$, makes sense. In addition, since $\gamma=\min _{i_{2}=1, \ldots, n_{2}}\left\{\gamma_{i_{2}}, \varphi\right\} \in(5-3 \alpha-2 \beta, 1)$, we have $\chi_{\alpha, \beta, \gamma}=$ $(\alpha+\beta+\gamma-2) / \alpha \in((3-2 \alpha-\beta) / \alpha, 1)$. Therefore, by virtue of the choice of the Hölder exponents $\eta_{i_{1}}$ and $\sigma_{i_{2}}$, the number $\tau=\min _{i_{l}=1, \ldots, n_{l}, l=1,2}\left\{\eta_{i_{1}}, \sigma_{i_{2}}, \chi_{\alpha, \beta, \gamma}\right\}$ belongs to $((3-2 \alpha-$ $\beta) / \alpha, 1)$ too, and the interval $I_{\alpha, \beta, \tau}$ is well defined. Further, the numbers $\eta, \tau_{1}$, and $\tau_{0}$ being as in the statements of Lemmas 42, 45, and 46, respectively, we have $\tau=\tau_{0} \leq \tau_{1} \leq \eta$. As a consequence, since $I_{\alpha, \beta, \tau} \subseteq I_{\alpha, \beta, \tau} \subseteq I_{\alpha, \beta, \eta}$ and $I_{\alpha, \beta, \tau} \subseteq J_{\alpha, \beta, \tau}$, all the mentioned lemmas are applicable with $\delta \in I_{\alpha, \beta, \tau}$. To this purpose, we stress that since $((3-2 \alpha-\beta) / \alpha, 1) \subseteq((2-\alpha-$ $\beta) / \alpha, 1)$ and $(5-3 \alpha-2 \beta, 1) \subseteq(4-2 \alpha-2 \beta, 1) \subseteq(3-2 \alpha-\beta, 1)$, the conditions for the applicability of both Lemmas 45 and 46 are fulfilled. Hence, now let $\delta \in I_{\alpha, \beta, \tau}$ being fixed. First, due to Lemma 42 , the operator $\widetilde{R}=\left.R\right|_{C_{0}^{\delta}\left(I_{T} ; X\right)}, \widetilde{R} g=R g$, $g \in C_{0}^{\delta}\left(I_{T} ; X\right)$, a fortiori maps $C_{0}^{\delta}\left(I_{T} ; X\right)$ into itself. Then, $C_{0}^{\delta}\left(I_{T} ; X\right)$ being endowed with the same norm $\|\cdot\|_{\delta, 0, T ; X}$ of $C^{\delta}\left(I_{T} ; X\right)$, from (197) we obtain the estimates

$$
\begin{array}{r}
\left\|\widetilde{R}^{n}\right\|_{\mathscr{L}\left(C_{0}^{\delta}\left(I_{T} ; X\right)\right)} \leq\left[c_{42}(T)\right]^{n}\left(\frac{T^{n}}{n !}\right)^{1 / p}, \quad n \in \mathbf{N} \cup\{0\}, \\
p \in\left(\frac{1}{1-2 \delta}, \infty\right) .
\end{array}
$$

In particular, (203) yields that $\sum_{n=0}^{\infty} \widetilde{R}^{n}$ converges in $\mathscr{L}\left(C_{0}^{\delta}\left(I_{T} ; X\right)\right)$. From generalized Neumann's Theorem it thus follows that $1 \in \rho(\widetilde{R})$, the inverse $(I-\widetilde{R})^{-1} \in \mathscr{L}\left(C_{0}^{\delta}\left(I_{T} ; X\right)\right)$ being precisely $\sum_{n=0}^{\infty} \widetilde{R}^{n}$. Since Lemmas 45 and 46 (both applied with (observe here that if $\mu \in\left[\delta+\mu_{\alpha, \beta}, 1\right.$ ), then the exponent $\widetilde{\nu}_{\alpha, \beta, \mu}$ in the last part of the proof of Lemma 46 satisfies $\widetilde{v}_{\alpha, \beta, \mu} \geq \widetilde{v}_{\alpha, \beta, \delta+\mu_{\alpha, \beta}} \geq \widetilde{v}_{\alpha, \beta, \delta+\varrho_{\alpha, \beta}}=\delta$. For, $\left.\nu_{\alpha, \beta, \delta+\mu_{\alpha, \beta}}=(\alpha \delta+1-\alpha) / \alpha=\delta+(1-\alpha) / \alpha\right) f \in C^{\mu}\left(I_{T} ; X\right)$, $\left.\mu \in\left[\delta+\mu_{\alpha, \beta}, 1\right) \subseteq\left[\delta+\rho_{\alpha, \beta}, 1\right)\right)$ imply that $w_{0}, w_{1} \in C_{0}^{\delta}\left(I_{T} ; X\right)$, we conclude that the fixed-point equation (179) admits the unique solution

$$
w=\sum_{n=0}^{\infty} \widetilde{R}^{n}\left(w_{0}+w_{1}\right) \in C_{0}^{\delta}\left(I_{T} ; X\right) .
$$

Observe now that the data vector $\left(k_{1}, \ldots, k_{n_{1}}, h_{1}, \ldots, h_{n_{2}}\right.$, $\left.f, y_{1}, \ldots, y_{n}, v_{1}+f(0)\right)$ satisfies all the assumptions which were needed to show the equivalence between the fixed-point equation (179) and problem (170). Indeed, $\delta \leq \tau$ and $\delta \leq$ $\delta+\mu_{\alpha, \beta} \leq \mu$ imply, respectively, that $k_{i_{1}} \in C^{\delta}\left(I_{T} ; Z\right), h_{i_{2}} \in$ $C^{\delta}\left(I_{T} ; \mathbf{C}\right)$ and $f \in C^{\delta}\left(I_{T} ; X\right), i_{l}=1, \ldots, n_{l}, l=1,2$, whereas, as in Lemma $46, \gamma=\min _{i_{2}=1, \ldots, n_{2}}\left\{\gamma_{i_{2}}, \varphi\right\}$ implies that $y_{i_{2}}, v_{1}+$ $f(0) \in Y_{\gamma}^{r}$. Therefore, since $A^{-1} \in \mathscr{L}(X)$, if $w \in C_{0}^{\delta}\left(I_{T} ; X\right)$ is the solution to the fixed-point equation (179), then $A^{-1} w \in$ $C_{0}^{\delta}\left(I_{T} ; X\right)$, too, and the function $F_{w}$ defined by (169) satisfies

$$
\begin{gathered}
F_{w} \in C^{\delta}\left(I_{T} ; X\right), \\
x_{0}=F_{w}(0)=\sum_{i_{2}=1}^{n_{2}} h_{i_{2}}(0) y_{i_{2}}+v_{1}+f(0) \in Y_{\gamma}^{r},
\end{gathered}
$$

where $\left.\delta \in I_{\alpha, \beta, \tau} \varsubsetneqq(2-\alpha-\beta) / \alpha, 1\right), \gamma \in(5-3 \alpha-2 \beta, 1) \varsubsetneqq(1-$ $\beta, 1)$, and $r \in[1, \infty]$. Consequently, recalling (168), we have proved that problem (160) has a unique strict global solution $v=L^{-1} w+v_{0} \in C^{\delta}\left(I_{T} ; \mathscr{D}(L)\right)$ satisfying $v(0)=L^{-1} w(0)+v_{0}=$ $v_{0}$ and such that $L v=w+L v_{0} \in C^{\delta}\left(I_{T} ; X\right)$. As far as the regularity of $D_{t} M v$ is concerned, instead, it suffices to observe that (168), (170), $w \in C_{0}^{\delta}\left(I_{T} ; X\right)$, and $F_{w} \in C^{\delta}\left(I_{T} ; X\right)$ yield

$$
D_{t} M v=D_{t} A^{-1} w=w+F_{w} \in C^{\delta}\left(I_{T} ; X\right) .
$$

The proof is complete.

Remark 49. Theorem 48 improves the faulty Thereoms 5.6 and 5.7 in [20] in two aspects. First, the assumption $3 \alpha+8 \beta>$ 10 is weakened to $5 \alpha+2 \beta>6$. In fact, $3 \alpha+8 \beta>10$ implies that $5 \alpha+2 \beta=3 \alpha+8 \beta+2 \alpha-6 \beta>10-4 \alpha \geq 6$. Hence, in the special case $\alpha=1$, the constraint $\beta>7 / 8$ in [20] reduces to the definitely weaker $\beta>1 / 2$. Second, in [20], only for $n_{1}=n_{2}=1$ and opportunely chosen $\gamma<$ $\beta$, the data $y_{1}$ and $v_{1}+f(0)$ were assumed to belong to the intermediate spaces $X_{A}^{\gamma, r}$, whereas here, removing the assumption $\gamma<\beta$ and considering the general case $n_{1}, n_{2} \epsilon$ $\mathbf{N}$, we allow $y_{1}, \ldots, y_{n_{2}}$ and $v_{1}+f(0)$ to belong also to the interpolation spaces $(X, \mathscr{D}(A))_{\gamma, r}$. To emphasize how much these aspects are decisive, let $\alpha=1$ in Theorem 48. Then, if $\beta \in(1 / 2,2 / 3]$ and the choice $X_{A}^{\psi, r}$ is understood for $Y_{\psi}^{r}$, we have $\gamma_{i_{2}}, \varphi \in(2-2 \beta, 1) \varsubsetneqq[\beta, 1)$, and the spaces $X_{A}^{\gamma_{i_{2}}, r}$ and $X_{A}^{\varphi, r}, i_{2}=1, \ldots, n_{2}$, may be smaller than $\mathscr{D}(A)$. However, the choice $Y_{\psi}^{r}=(X, \mathscr{D}(A))_{\psi, r}$ being admissible, in this situation too we can solve problem (160) with the data in spaces larger than $\mathscr{D}(A)$. Further, since $2 / 3<7 / 8$, in this case the results in [20] would not be applicable. These observations lead us to conclude that the more delicate approach followed in this paper with respect to that in [20, Sections 4 and 5], and especially the sharper results of the present Sections 3 and 4 , yield a valuable refinement in the treatment of questions of maximal time regularity for the strict solutions to (160); of course, unless that the not too much significant case $\beta=1$ is assumed in (H2).

Remark 50. The assumption $5 \alpha+2 \beta>6$ in (H2) implies that $\beta \in((6-5 \alpha) / 2, \alpha] \subseteq(1 / 2,1]$ and $\alpha \in(6 / 7,1]$. In particular, if $\alpha=1$, then Theorem 48 holds with $\beta \in(1 / 2,1], \eta_{i_{1}}, \sigma_{i_{2}} \in$ $(1-\beta, 1), \gamma_{i_{2}}, \varphi \in(2-2 \beta, 1), i_{l}=1, \ldots, n_{l}, l=1,2$, and $\mu_{1, \beta}=1-\beta$. Hence, $\gamma \in(2-2 \beta, 1), \chi_{1, \beta, \gamma}=\beta+\gamma-1 \in(1-\beta, \beta)$, and $\delta \in I_{1, \beta, \tau}$ with $\tau \in(1-\beta, \beta)$, where

$$
\begin{array}{r}
I_{1, \beta, \tau}=(1-\beta, \tau], \quad \text { if } \tau \in\left(1-\beta, \frac{1}{2}\right), \\
I_{1, \beta, \tau}=\left(1-\beta, \frac{1}{2}\right), \quad \text { if } \tau \in\left[\frac{1}{2}, \beta\right) .
\end{array}
$$

Clearly, if $\beta=1$, then $5 \alpha+2 \beta>6$ is redundant, and Theorem 48 holds with $\eta_{i_{1}}, \sigma_{i_{2}}, \gamma_{i_{2}}, \varphi \in(0,1), i_{l}=$ $1, \ldots, n_{l}, l=1,2, \mu_{1,1}=0, \gamma=\chi_{1,1, \gamma} \in(0,1)$, and $\delta \in I_{1,1, \tau}$, $\tau \in(0,1)$, where $I_{1,1, \tau}=(0, \tau]$ if $\tau \in(0,1 / 2)$ and $I_{1,1, \tau}=$ $(0,1 / 2)$ if $\tau \in[1 / 2,1)$. 
Remark 51. Observe that, if the $\eta_{i_{1}}$ 's and $\sigma_{i_{2}}$ 's are assumed to vary in the smaller interval $U_{\alpha, \beta}:=((3-2 \alpha-\beta) / \alpha,(\alpha+$ $\beta-1) / \alpha$ ), then $\varphi$ and the $\gamma_{i_{2}}$ 's can be chosen such that $\tau=\min _{i_{1}=1, \ldots, n_{l}, l=1,2}\left\{\eta_{i_{1}}, \sigma_{i_{2}}\right\}$. To this purpose, letting $\rho=$ $\max _{i_{1}=1, \ldots, n_{l}, l=1,2}\left\{\eta_{i_{1}}, \sigma_{i_{2}}\right\} \in U_{\alpha, \beta}$, it suffices to take $\gamma_{i_{2}}, \varphi \in$ $V_{\alpha, \beta, \rho}, i_{2}=1, \ldots, n_{2}$, where $V_{\alpha, \beta, \rho}:=[2+\alpha \rho-\alpha-\beta, 1) \varsubsetneqq$ $(5-3 \alpha-2 \beta, 1)$. Then $\gamma=\min _{i_{2}=1, \ldots, n_{2}}\left\{\gamma_{i_{2}}, \varphi\right\} \in V_{\alpha, \beta, \rho}$ and $\chi_{\alpha, \beta, \gamma}=(\alpha+\beta+\gamma-2) / \alpha \geq \rho$. In other words, provided that the data vector $\left(y_{1}, \ldots, y_{n_{2}}, v_{1}+f(0)\right)$ is smooth enough, the pair $\left(L v, D_{t} M v\right)$ has the maximal time regularities which is the minimal between the time regularities of the $k_{i_{1}}$ 's and $h_{i_{2}}$ 's.

We conclude with the results which follow from Theorem 48 for problems (163)-(166).

Theorem 52. Assume (161) and $v_{0} \in \mathscr{D}(L)$, and let $5 \alpha+2 \beta>6$ in (H2). Assume that $k_{i_{1}} \in C^{\eta_{i_{1}}}\left(I_{T} ; Z\right)$ and $L v_{0}+f(0) \in Y_{\gamma}^{r}$, where $\eta_{i_{1}} \in((3-2 \alpha-\beta) / \alpha, 1), i_{1}=1, \ldots, n_{1}, \gamma \in(5-3 \alpha-$ $2 \beta, 1)$, and $r \in[1, \infty]$. Let $\tau=\min _{i_{1}=1, \ldots, n_{1}}\left\{\eta_{i_{1}}, \chi_{\alpha, \beta, \gamma}\right\}$, where $\chi_{\alpha, \beta, \gamma}=(\alpha+\beta+\gamma-2) / \alpha$. Then, for every fixed $\delta \in I_{\alpha, \beta, \tau}$ problem (165) admits a unique strict solution $v \in C^{\delta}\left(I_{T} ; \mathscr{D}(L)\right)$ satisfying $v(0)=v_{0}$ and such that $L v, D_{t} M v \in C^{\delta}\left(I_{T} ; X\right)$, provided that $f \in C^{\mu}\left(I_{T} ; X\right), \mu \in\left[\delta+\mu_{\alpha, \beta}, 1\right), \mu_{\alpha, \beta}=$ $(3-2 \alpha-\beta) / \alpha$.

Proof. Repeat the proofs of Lemmas 42, 45, and 46, Corollary 44, and Theorem 48, letting there $\lambda_{0}=h_{i_{2}}=0$, $i_{2}=1, \ldots, n_{2}$. To this purpose, observe that (169) and (174) reduce to $F_{w}(t)=\sum_{i_{1}=1}^{n_{1}}\left[\mathscr{K}\left(k_{i_{1}}, S_{i_{1}} w\right)(t)+\mathscr{K}\left(k_{i_{1}}, L_{i_{1}} v_{0}\right)(t)\right]+$ $L v_{0}+f(t)$ and $x_{0}=L v_{0}+f(0)$. Consequently, (180)-(182) change to $w_{0}=Q_{7} x_{0}+\sum_{i_{1}=1}^{n_{1}} Q_{6}\left(k_{i_{1}}, L_{i_{1}} v_{0}\right)+Q_{5} \widetilde{f}, w_{1}=$ $-\sum_{i_{1}=1}^{n_{1}} Q_{3}\left(k_{i_{1}}, L_{i_{1}} v_{0}\right)-Q_{2} f$, and $R w=\sum_{i_{1}=1}^{n_{1}}\left[Q_{6}\left(k_{i_{1}}, S_{i_{1}} w\right)-\right.$ $\left.Q_{3}\left(k_{i_{1}}, S_{i_{1}} w\right)\right]$.

Theorem 53. Assume (161) and $v_{0} \in \mathscr{D}(L)$, and let $5 \alpha+2 \beta>6$ in (H2). Assume that $k_{i_{1}} \in C^{\eta_{i_{1}}}\left(I_{T} ; Z\right), h_{i_{2}} \in C^{\sigma_{i_{2}}}\left(I_{T} ; \mathbf{C}\right), y_{i_{2}} \in$ $Y_{\gamma_{i_{2}}}^{r}$, and $L v_{0} \in Y_{\varphi}^{r}$, where $\eta_{i_{1}}, \sigma_{i_{2}} \in((3-2 \alpha-\beta) / \alpha, 1), \gamma_{i_{2}}, \varphi \in$ $(5-3 \alpha-2 \beta, 1), i_{l}=1, \ldots, n_{l}, l=1,2$, and $r \in[1, \infty]$. Let $\gamma=\min _{i_{2}=1, \ldots, n_{2}}\left\{\gamma_{i_{2}}, \varphi\right\}$ and $\tau=\min _{i_{l}=1, \ldots, n_{l}, l=1,2}\left\{\eta_{i_{1}}, \sigma_{i_{2}}, \chi_{\alpha, \beta, \gamma}\right\}$, where $\chi_{\alpha, \beta, \gamma}=(\alpha+\beta+\gamma-2) / \alpha$. Then, for every fixed $\delta \in I_{\alpha, \beta, \tau}$ problem (166) admits a unique strict solution $v \in C^{\delta}\left(I_{T} ; \mathscr{D}(L)\right)$ satisfying $v(0)=v_{0}$ and such that $L v, D_{t} M v \in C^{\delta}\left(I_{T} ; X\right)$.

Proof. Let $\lambda_{0}=f=0$ in the proofs of Lemmas 42, 45, and 46, Corollary 44, and Theorem 48. In this case, (169) and (174) reduce to $F_{w}(t)=\sum_{i_{1}=1}^{n_{1}}\left[\mathscr{K}\left(k_{i_{1}}, S_{i_{1}} w\right)(t)+\mathscr{K}\left(k_{i_{1}}, L_{i_{1}} v_{0}\right)(t)\right]+$ $\sum_{i_{2}=1}^{n_{2}} h_{i_{2}}(t) y_{i_{2}}+L v_{0}$ and $x_{0}=\sum_{i_{2}=1}^{n_{2}} h_{i_{2}}(0) y_{i_{2}}+L v_{0}$. Hence, (180)-(182) change to $w_{0}=Q_{7} x_{0}+\sum_{i_{1}=1}^{n_{1}} Q_{6}\left(k_{i_{1}}, L_{i_{1}} v_{0}\right)+$ $\sum_{i_{2}=1}^{n_{2}} Q_{5} \widetilde{h}_{i_{2}}, w_{1}=-\sum_{i_{1}=1}^{n_{1}} Q_{3}\left(k_{i_{1}}, L_{i_{1}} v_{0}\right)-\sum_{i_{2}=1}^{n_{2}} Q_{4}\left(h_{i_{2}}, y_{i_{2}}\right)$, and $R w=\sum_{i_{1}=1}^{n_{1}}\left[Q_{6}\left(k_{i_{1}}, S_{i_{1}} w\right)-Q_{3}\left(k_{i_{1}}, S_{i_{1}} w\right)\right]$.

Let us now turn to the degenerate differential problems (163) and (164).
Theorem 54. Assume (161) and $v_{0} \in \mathscr{D}(L)$, and let $5 \alpha+2 \beta>6$ in (H2). Assume that $L v_{0}+f(0) \in Y_{\gamma}^{r}, \gamma \in(5-3 \alpha-2 \beta, 1)$, $r \in[1, \infty]$, and let $\chi_{\alpha, \beta, \gamma}=(\alpha+\beta+\gamma-2) / \alpha$. Then, for every fixed $\delta \in I_{\alpha, \beta, \chi_{\alpha, \beta, \gamma}}$ problem (163) admits a unique strict global solution $v \in C^{\delta}\left(I_{T} ; \mathscr{D}(L)\right)$ satisfying $v(0)=v_{0}$ and such that $L v, D_{t} M v \in C^{\delta}\left(I_{T} ; X\right)$, provided that $f \in C^{\mu}\left(I_{T} ; X\right), \mu \in[\delta+$ $\left.\mu_{\alpha, \beta}, 1\right), \mu_{\alpha, \beta}=(3-2 \alpha-\beta) / \alpha$.

Proof. Let $\lambda_{0}=k_{i_{1}}=h_{i_{2}}=0, i_{l}=1, \ldots, n_{l}, l=1,2$, in problem (160) and formulae (169), (174) and, (179)-(182). Then, $F_{w}(t)=L v_{0}+f(t), x_{0}=L v_{0}+f(0)$ and $w=$ $w_{0}+w_{1}=Q_{7} x_{0}+Q_{5} \tilde{f}-Q_{2} f$. Consequently, Lemma 42 and Corollary 44 are unneeded, and the proof of Theorem 48 simplifies as follows. First, due to $\gamma \in(5-3 \alpha-2 \beta, 1)$ we have $\chi_{\alpha, \beta, \gamma} \in((3-2 \alpha-\beta) / \alpha, 1)$, and the interval $I_{\alpha, \beta, \chi_{\alpha, \beta, \gamma}}$ is well defined. Hence, let $\delta \in I_{\alpha, \beta, \chi_{\alpha, \beta, \gamma}}$ being fixed. Since (cf. (200)) $f \in C^{\mu}\left(I_{T} ; X\right), \mu \in\left[\delta+\mu_{\alpha, \beta}, 1\right) \varsubsetneqq((3-2 \alpha-\beta) / \alpha, 1)$, reasoning as in the last part of the proof of Lemma 45 we get $Q_{2} f \in C_{0}^{\delta}\left(I_{T} ; X\right)$. Moreover (see the proof of Lemma 46), since $x_{0} \in Y_{\gamma}^{r}, \gamma \in(5-3 \alpha-2 \beta, 1) \subseteq(2-\alpha-\beta, 1)$ and $\tilde{f} \in C_{0}^{\mu}\left(I_{T} ; X\right), \mu \in\left[\delta+\mu_{\alpha, \beta}, 1\right) \subseteq\left[\delta+\varrho_{\alpha, \beta}, 1\right)$, $\varrho_{\alpha, \beta}=(2-\alpha-\beta) / \alpha$, Corollary 38 and Lemma 32 applied with $\left(x, \delta_{7}\right)=\left(x_{0}, \delta\right)$ and $\left(g_{5}, \delta_{5}\right)=\left(\tilde{f}, \delta+\varrho_{\alpha, \beta}\right)$ yield $Q_{7} x_{0}, Q_{5} \tilde{f} \in$ $C_{0}^{\delta}\left(I_{T} ; X\right)$. Summing up, we find that $w \in C_{0}^{\delta}\left(I_{T} ; X\right)$. The assertion then follows from $v=L^{-1} w+v_{0}$ and (cf. (206)) $D_{t} M v=w+L v_{0}+f$.

Remark 55. We refer to [19, Theorem 5.3] for a result of both time and space regularity for problem (163). There, provided that $\psi$ and $\delta$ are opportunely chosen and the data satisfy assumptions similar to those in Theorem 54, it is shown that $D_{t} M v \in C^{\delta}\left(I_{T} ;(X, \mathscr{D}(A))_{\psi, r}\right)$, and that the higher is the order $\psi$ of the interpolation space where we look for space regularity, the lower is the Hölder exponent $\delta$ of regularity in time. Notice that $L v=D_{t} M v-f$ has no space regularity, unless $f$ has too.

Theorem 56. Assume (161) and $v_{0} \in \mathscr{D}(L)$, and let $5 \alpha+2 \beta>6$ in (H2). Assume that $h_{i_{2}} \in C^{\sigma_{i_{2}}}\left(I_{T} ; \mathbf{C}\right), y_{i_{2}} \in Y_{\gamma_{i_{2}}}^{r}$, and $L v_{0} \in$ $Y_{\varphi}^{r}$, where $\sigma_{i_{2}} \in((3-2 \alpha-\beta) / \alpha, 1), \gamma_{i_{2}}, \varphi \in(5-3 \alpha-2 \beta, 1)$, $i_{2}=1, \ldots, n_{2}$, and $r \in[1, \infty]$. Let $\gamma=\min _{i_{2}=1, \ldots, n_{2}}\left\{\gamma_{i_{2}}, \varphi\right\}$ and $\tau=\min _{i_{2}=1, \ldots, n_{2}}\left\{\sigma_{i_{2}}, \chi_{\alpha, \beta, \gamma}\right\}$, where $\chi_{\alpha, \beta, \gamma}=(\alpha+\beta+\gamma-2) / \alpha$. Then, for every fixed $\delta \in I_{\alpha, \beta, \tau}$, problem (164) admits a unique strict global solution $v \in C^{\delta}\left(I_{T} ; \mathscr{D}(L)\right)$ satisfying $v(0)=v_{0}$ and such that $L v, D_{t} M v \in C^{\delta}\left(I_{T} ; X\right)$.

Proof. Let $\lambda_{0}=k_{i_{1}}=f=0, i_{1}=1, \ldots, n_{1}$, in problem (160) and formulae (169), (174), and (179)-(182). Then, $F_{w}(t)=$ $\sum_{i_{2}=1}^{n_{2}} h_{i_{2}}(t) y_{i_{2}}+L v_{0}, x_{0}=\sum_{i_{2}=1}^{n_{2}} h_{i_{2}}(0) y_{i_{2}}+L v_{0}$ and $w=$ $w_{0}+w_{1}=Q_{7} x_{0}+\sum_{i_{2}=1}^{n_{2}} Q_{5} \widetilde{h}_{i_{2}}-\sum_{i_{2}=1}^{n_{2}} Q_{4}\left(h_{i_{2}}, y_{i_{2}}\right)$. Therefore, as in Theorem 54, we do not need Lemma 42 and Corollary 44, and the proof of Theorem 48 simplifies as follows. Again, $\gamma=\min _{i_{2}=1, \ldots, n_{2}}\left\{\gamma_{i_{2}}, \varphi\right\} \in(5-3 \alpha-2 \beta, 1)$ implies that 
$\chi_{\alpha, \beta, \gamma} \in((3-2 \alpha-\beta) / \alpha, 1)$, so that $\tau=\min _{i_{2}=1, \ldots, n_{2}}\left\{\sigma_{i_{2}}, \chi_{\alpha, \beta, \gamma}\right\} \in$ $((3-2 \alpha-\beta) / \alpha, 1)$, and the interval $I_{\alpha, \beta, \tau}$ is well defined. Let $\delta \in I_{\alpha, \beta, \tau}$ be fixed. First (see the proof of Lemma 45), since $\gamma_{i_{2}} \in(5-3 \alpha-2 \beta, 1) \subseteq(3-2 \alpha-\beta, 1)$, Lemma 30 applied with $\left(g_{4}, y, \delta_{4}, \gamma\right)=\left(h_{i_{2}}, y_{i_{2}}, \delta, \gamma_{i_{2}}\right)$ yields $Q_{4}\left(h_{i_{2}}, y_{i_{2}}\right) \in C_{0}^{\delta}\left(I_{T} ; X\right)$, $i_{2}=1, \ldots, n_{2}$. On the other side (see the proof of Lemma 46), since $x_{0} \in Y_{\gamma}^{r}$ and $\tilde{h}_{i_{2}} \in C_{0}^{\delta}\left(I_{T} ; Y_{\gamma_{i_{2}}}^{r}\right) \hookrightarrow C_{0}^{\delta}\left(I_{T} ; Y_{\gamma}^{r}\right), \gamma \in(5-$ $3 \alpha-2 \beta, 1) \subseteq(2-\alpha-\beta, 1)$, from Lemma 37 and Corollary 38 applied with $\left(g_{5}, \delta_{5}\right)=\left(\widetilde{h}_{i_{2}}, \delta\right)$ and $\left(x, \delta_{7}\right)=\left(x_{0}, \delta\right)$ we deduce that $Q_{5} \widetilde{h}_{i_{2}}, Q_{7} x_{0} \in C_{0}^{\delta}\left(I_{T} ; X\right), i_{2}=1, \ldots, n_{2}$. Summing up, we find that $w \in C_{0}^{\delta}\left(I_{T} ; X\right)$, and the assertion again follows from $v=L^{-1} w+v_{0}$ and (cf. (206)) $D_{t} M v=w+L v_{0}+$ $\sum_{i_{2}=1}^{n_{2}} h_{i_{2}} y_{i_{2}}$.

\section{An Application to a Concrete Case}

Theorem 48 is here applied to determine the right functional framework where to search for the solution of an inverse problem arising in the theory of heat conduction for materials with memory. To this purpose, let $\Omega \varsubsetneqq \mathbf{R}^{N}, N \in \mathbf{N}$, be a bounded domain with boundary $\partial \Omega$ of class $C^{1,1}$ (cf. [36, p. 94]). If $\Omega$ represents a rigid thermal body with memory, then the linearized theory of heat flow yields the following equations linking the internal energy $e$, the heat flux $\mathbf{q}=$ $\left(q_{1}, \ldots, q_{N}\right)$, and the temperature $\Theta$ (cf. $\left.[32,37-40]\right)$ :

$$
\begin{gathered}
e(t, x)=e_{0}+a(0, x) \Theta(t, x)+\int_{0}^{t} D_{t} a(t-s, x) \Theta(s, x) \mathrm{d} s \\
q_{j}(t, x)=-\sum_{i=1}^{r_{1}} b_{i}(0) C_{i, j}\left(x ; D_{x}\right) \Theta(t, x) \\
-\sum_{i=1}^{r_{1}} \int_{0}^{t} D_{t} b_{i}(t-s) C_{i, j}\left(x ; D_{x}\right) \Theta(s, x) \mathrm{d} s \\
j=1, \ldots, N \\
D_{t} e(t, x)=-\operatorname{div}_{x} \mathbf{q}(t, x)+g(t, x) \\
=-\sum_{j=1}^{N} D_{x_{j}} q_{j}(t, x)+g(t, x) .
\end{gathered}
$$

Here $t \in I_{T}, I_{T}=[0, T], T>0, x=\left(x_{1}, \ldots, x_{N}\right) \in \Omega, r_{1} \in \mathbf{N}$, $e_{0} \in \mathbf{R}$, and $D_{t}=\partial / \partial t$, whereas the $C_{i, j}\left(x ; D_{x}\right)^{\prime}$ s represent the first-order linear differential operators

$$
\begin{array}{r}
C_{i, j}\left(\mathrm{x} ; D_{x}\right)=\sum_{k=1}^{N} c_{i, j, k}(x) D_{x_{k}}, \quad x \in \bar{\Omega}, \\
i=1, \ldots, r_{1}, \quad j=1, \ldots, N,
\end{array}
$$

where $c_{i, j, k} \in C^{1}(\bar{\Omega} ; \mathbf{R})$ and $D_{x_{k}}=\partial / \partial x_{k}, i=1, \ldots, r_{1}$, $j, k=1, \ldots, N$. According to the terminology of $[39,40]$, the functions $a, b_{i}, i=1, \ldots, r_{1}$, and $g$ are called, respectively, the energy-temperature relaxation function, the heat conduction relaxation functions, and the heat supply function and we assume that they satisfy the following conditions:

$$
\begin{aligned}
& D_{t}^{k} a(\cdot, x) \in C\left(I_{T} ; \mathbf{R}\right), \quad k=0,1,2, \\
& a(0, x) \geq 0, \quad x \in \Omega, \\
D_{t}^{k} b_{i} & \in C\left(I_{T} ; \mathbf{R}\right), \quad k=0,1, \quad i=1, \ldots, r_{1}, \\
& g \in C^{1}\left(I_{T} \times \Omega ; \mathbf{R}\right) .
\end{aligned}
$$

Notice that, different from [32, 37-40], here the energytemperature relaxation function $a$ is assumed to depend also on the spatial variable $x \in \Omega$. In physical terms, this is equivalent to say that $\Omega$ represents a rigid inhomogeneous material with memory. Furthermore, in contrast with the quoted papers where only the cases $r_{1}=1$ and $C_{1, j}\left(x ; D_{x}\right)=$ $D_{x_{j}}$ are treated, here we have assumed that the history record of $\Omega$ is kept by an arbitrary number $r_{1} \in \mathbf{N}$ of heat conduction relaxation functions and that the $C_{i, j}$ 's are the more general first-order differential operators defined in (209).

By setting

$$
\tilde{a}_{j, k}=\sum_{i=1}^{r_{1}} b_{i}(0) c_{i, j, k} \in C^{1}(\bar{\Omega} ; \mathbf{R}), \quad j, k=1, \ldots, N
$$

from (208) and (209), it thus follows that the temperature $\Theta$ must satisfy the following equation:

$$
\begin{aligned}
a(0, x) & D_{t} \Theta(t, x)+D_{t} a(0, x) \Theta(t, x) \\
& +\int_{0}^{t} D_{t}^{2} a(t-s, x) \Theta(s, x) \mathrm{d} s-g(t, x) \\
= & \sum_{j, k=1}^{N} D_{x_{j}}\left[\tilde{a}_{j, k}(x) D_{x_{k}} \Theta(t, x)\right] \\
& +\sum_{i=1}^{r_{1}} \int_{0}^{t} D_{t} b_{i}(t-s) \sum_{j=1}^{N} D_{x_{j}} C_{i, j}\left(x ; D_{x}\right) \Theta(s, x) \mathrm{d} s .
\end{aligned}
$$

Let us now assume that $a$ is of the following special form:

$$
a(t, x)=\sum_{n=1}^{2} m_{n}(x) u_{n}(t), \quad(t, x) \in I_{T} \times \Omega,
$$

where the functions $m_{n}$ and $u_{n}, n=1,2$, satisfy the following conditions (cf. (210)):

$$
\begin{gathered}
m_{n} \in L_{\infty}(\Omega), \quad n=1,2, \\
m_{1} \geq 0, \quad m_{2}>0, \\
u_{n} \in C^{2}\left(I_{T} ; \mathbf{R}\right), \quad n=1,2, \\
u_{2}(0)=0, \quad u_{1}(0)>0, \quad D_{t} u_{2}(0)>0 .
\end{gathered}
$$


Here, $L_{q}(\Omega)=L_{q}(\Omega ; \mathbf{R}), q \in[1, \infty]$, is the usual $L_{q}$ space with norm $\|\cdot\|_{q ; \Omega}\left(\right.$ cf. [36, Chapter 7]). Using $m_{2}, u_{1}(0)$, $D_{t} u_{2}(0)>0$, for $t \in I_{T}$ and $x \in \Omega$ we now set

$$
\begin{aligned}
& a_{0}(x)=-\left[u_{1}(0)\right]^{-1} m_{2}(x) D_{t} u_{2}(0)<0, \\
& a_{j, k}(x)=\left[u_{1}(0)\right]^{-1} \tilde{a}_{j, k}(x), \quad j, k=1, \ldots, N, \\
& L\left(x ; D_{x}\right)=\sum_{j, k=1}^{N} D_{x_{j}}\left[a_{j, k}(x) D_{x_{k}}\right]+a_{0}(x), \\
& L_{i}\left(x ; D_{x}\right)=\left[u_{1}(0)\right]^{-1} \sum_{j=1}^{N} D_{x_{j}} C_{i, j}\left(x ; D_{x}\right), \\
& i=1, \ldots, r_{1} \text {, } \\
& L_{r_{1}+n}\left(x ; D_{x}\right)=L_{r_{1}+n}(x)=\left[u_{1}(0)\right]^{-1} m_{n}(x) \text {, } \\
& n=1,2 \text {, } \\
& k_{i}(t)=D_{t} b_{i}(t), \quad i=1, \ldots, r_{1}, \\
& k_{r_{1}+n}(t)=-D_{t}^{2} u_{n}(t), \quad n=1,2, \\
& \widetilde{g}(t, x)=\left[u_{1}(0)\right]^{-1} g(t, x), \\
& \lambda_{0}=-\left[u_{1}(0)\right]^{-1} D_{t} u_{1}(0) \in \mathbf{R} .
\end{aligned}
$$

Then, since (214)-(216) yield $a(0, x)=m_{1}(x) u_{1}(0)$ and $D_{t}^{k} a(t, x)=\sum_{n=1}^{2} m_{n}(x) D_{t}^{k} u_{n}(t), k=1,2$, if we multiply both sides of (213) by $\left[u_{1}(0)\right]^{-1}$ and use (218)-(223), we are led to the following basic differential equation for the temperature $\Theta$, where $n_{1}=r_{1}+2$ :

$$
\begin{aligned}
& D_{t}\left[m_{1}(x) \Theta(t, x)\right] \\
&= \lambda_{0} m_{1}(x) \Theta(t, x)+L\left(x ; D_{x}\right) \Theta(t, x)+\widetilde{g}(t, x) \\
&+\sum_{i=1}^{n_{1}} \int_{0}^{t} k_{i}(t-s) L_{i}\left(x ; D_{x}\right) \Theta(s, x) \mathrm{d} s, \\
& t \in I_{T}, \quad x \in \Omega .
\end{aligned}
$$

We endow this differential equation with the initial condition $\Theta(0, x)=\Theta_{0}(x), x \in \Omega$, and the Dirichlet boundary condition $\Theta(t, x)=0, t \in I_{T}, x \in \partial \Omega$.

We now suppress the dependence on $x \in \Omega$, and we transform (224) in a degenerate integrodifferential Cauchy problem in a Banach space $X$. To this purpose, for every fixed $q \in(1, \infty)$ and observing that $m_{n} \in L_{\infty}(\Omega)$ implies that $\left\|m_{n} u\right\|_{q ; \Omega} \leq\left\|m_{n}\right\|_{\infty ; \Omega}\|u\|_{q ; \Omega}$ for every $u \in L_{q}(\Omega), n=1,2$, we set

$$
\begin{gathered}
X=\mathscr{D}(M)=\mathscr{D}\left(L_{r_{1}+n}\right)=L_{q}(\Omega), \quad n=1,2, \\
\mathscr{D}(L)=W_{q}^{2}(\Omega) \cap \dot{W}_{q}^{1}(\Omega), \quad \mathscr{D}\left(L_{i}\right)=W_{q}^{2}(\Omega), \\
i=1, \ldots, r_{1}, \\
M, L_{r_{1}+n} \in \mathscr{L}(X), \quad M u=m_{1} u, \\
L_{r_{1}+n} u=L_{r_{1}+n}(x) u, \quad u \in X, n=1,2, \\
L: \mathscr{D}(L) \subseteq X \longrightarrow X, \\
L u=L\left(x ; D_{x}\right) u, u \in \mathscr{D}(L), \\
L_{i}: \mathscr{D}\left(L_{i}\right) \subseteq X \longrightarrow X, \\
L_{i} u=L_{i}\left(x ; D_{x}\right) u, \quad u \in \mathscr{D}\left(L_{i}\right), \quad i=1, \ldots, r_{1} .
\end{gathered}
$$

Here (cf. [36, Chapter 7]), $W_{q}^{k}(\Omega)=W_{q}^{k}(\Omega ; \mathbf{R}), k \in \mathbf{N} \cup\{0\}$, $q \in(1, \infty)$, denotes the usual Sobolev space endowed with the norm $\|\cdot\|_{k, q ; \Omega}\left(\left(W_{q}^{0}(\Omega),\|\cdot\|_{0, q ; \Omega}\right)=\left(L_{q}(\Omega),\|\cdot\|_{q ; \Omega}\right)\right)$, whereas $\stackrel{\circ}{W}_{q}^{k}(\Omega)$ denotes the completion of $C_{0}^{\infty}(\Omega ; \mathbf{R})$ in $W_{q}^{k}(\Omega), C_{0}^{\infty}(\Omega ; \mathbf{R})$ being the set of all real-valued infinitely differentiable functions having compact support in $\Omega$. We further assume that there exists positive constant $\Lambda_{i}, i=$ $0, \ldots, r_{1}$, such that for every $(x, \xi) \in \bar{\Omega} \times \mathbf{R}^{N}$ the following inequalities hold:

$$
\begin{gathered}
\sum_{j, k=1}^{N} c_{i, j, k}(x) \xi_{j} \xi_{k} \geq \Lambda_{i}|\xi|^{2}, \quad i=1, \ldots, r_{1}, \\
\sum_{j, k=1}^{r_{1}} b_{i}(0) \Lambda_{i} \geq \Lambda_{0},
\end{gathered}
$$

where $|\xi|^{2}=\sum_{l=1}^{N} \xi_{l}^{2}$. Therefore, from (212), (218), and (230) we get

$$
\begin{aligned}
\sum_{j, k=1}^{N} a_{j, k}(x) \xi_{j} \xi_{k} & =\left[u_{1}(0)\right]^{-1} \sum_{i=1}^{r_{1}} b_{i}(0) \sum_{j, k=1}^{N} c_{i, j, k} \xi_{j} \xi_{k} \\
& \geq\left[u_{1}(0)\right]^{-1} \Lambda_{0}|\xi|^{2}
\end{aligned}
$$

From (225)-(231) it follows that $M, L$, and $L_{i}, i=1, \ldots, n_{1}$, are closed linear operators from $X$ to itself, and the relation $\mathscr{D}(L) \subsetneq \bigcap_{i=1}^{n_{1}}\left[\mathscr{D}(M) \cap \mathscr{D}\left(L_{i}\right)\right]=W_{q}^{2}(\Omega)$ holds. In addition, due to (212), (217), (218), and (231), from [36, Theorem 9.15 and Lemma 9.17], it follows that for every fixed $q \in(1, \infty)$ the operator $L$ admits an inverse operator $L^{-1} \in \mathscr{L}\left(X ; W_{q}^{2}(\Omega)\right)$. Hence, a fortiori, $L^{-1} \in \mathscr{L}(X)$ and so condition (161) is satisfied (observe also that $L^{-1} \in$ $\mathscr{L}\left(X ; W_{q}^{2}(\Omega)\right)$ implies that the norms $\|\cdot\|_{2, q ; \Omega}$ and $\|\cdot\|_{\mathscr{D}(L)}=$ $\|\cdot\|_{q ; \Omega}+\|L \cdot\|_{q ; \Omega}$ are equivalent on $\mathscr{D}(L)$. In fact, if $v \in \mathscr{D}(L)$, 
then $\|v\|_{2, q ; \Omega}=\left\|L^{-1} L v\right\|_{2, q ; \Omega} \leq\left\|L^{-1}\right\|_{\mathscr{L}\left(X ; W_{q}^{2}(\Omega)\right)}\|v\|_{\mathscr{D}(L)} \leq$ $\widetilde{C}\left\|L^{-1}\right\|_{\mathscr{L}\left(X ; W_{q}^{2}(\Omega)\right)}\|v\|_{2, q ; \Omega}, \widetilde{C}$ being a positive constant depending on $\max _{j, k=1, \ldots, N}\left\|a_{j, k}\right\|_{C^{1}(\bar{\Omega} ; \mathbf{R})}$ ). The closed graph theorem then yield $M L^{-1}, L_{i} L^{-1} \in \mathscr{L}(X), i=1, \ldots, n_{1}$. Moreover (cf. [19, formula (77)], and [41, formula (2.16)]), the following estimate holds (of course, here $X=L_{q}(\Omega ; \mathbf{R})$ is replaced with the more general $\left.X=L_{q}(\Omega ; \mathbf{C})\right)$ :

$$
\left\|M(\lambda M-L)^{-1}\right\|_{\mathscr{L}(X)} \leq C(|\lambda|+1)^{-\beta}, \quad \forall \lambda \in \Sigma_{1}, \quad \beta=\frac{1}{q},
$$

where $\Sigma_{1}=\{z \in \mathbf{C}: \mathfrak{R e} z \geq-c(|\Im \mathrm{m} z|+1), \mathfrak{I m} z \in \mathbf{R}\}, c$ being a suitable positive constant depending on $q$ and $\left\|m_{1}\right\|_{\infty ; \Omega}$. Hence, condition (H2) is satisfied with $X=L^{q}(\Omega)$ and $(\alpha, \beta)=(1,1 / q)$. Notice that, since $m_{1}$ may have zeros in $\Omega, M^{-1}$ is in general a m. l. operator, so that $A=L M^{-1}$ is determined by (cf. (162)):

$$
\begin{gathered}
\mathscr{D}(A)=M(\mathscr{D}(L))=\left\{m_{1} v: v \in \mathscr{D}(L)\right\}, \\
A u=\left\{L v: v \in \mathscr{D}(L) \text { such that } u=m_{1} v\right\}, \quad u \in \mathscr{D}(A) .
\end{gathered}
$$

Using the convolution operator $\mathscr{K}$ in (104) in which for the bilinear operator $\mathscr{P}$ we take the scalar multiplication in $X$, from (224)-(229) we finally obtain that the temperature $\Theta(t)=\Theta(t, \cdot)$ solves the following degenerate integrodifferential Cauchy problem in $X$ :

$$
\begin{gathered}
D_{t}(M \Theta(t))=\left[\lambda_{0} M+L\right] \Theta(t) \\
+\sum_{i=1}^{n_{1}} \mathscr{K}\left(k_{i}, L_{i} \Theta\right)(t)+\widetilde{g}(t), \quad t \in I_{T}, \\
\Theta(0)=\Theta_{0} .
\end{gathered}
$$

Now, assume for a moment that we are interested in solving the inverse problem of recovering both the temperature $\Theta$ and the memory kernels $k_{1}, \ldots, k_{r_{1}}$ in (234). Clearly, due to (222), if we recover $k_{1}, \ldots, k_{r_{1}}$, then the heat conduction relaxation functions $b_{1}, \ldots, b_{r_{1}}$ will be known too, unless of the $r_{1}$ arbitrary constants $b_{i}(0), i=1, \ldots, r_{1}$. Indeed, $b_{1}(t)=$ $b_{i}(0)+\int_{0}^{t} k_{i}(s) \mathrm{d} s, t \in I_{T}$. To solve such an inverse problem, we need $r_{1}$ additional informations other than the initial condition $\Theta(0)=\Theta_{0}$, which, in general, suffices only to guarantee the well-posedness of the direct problem of recovering $\Theta$ in (234). Suppose then that the following additional pieces of information are given:

$$
\Psi_{j}[M \Theta(t)]=g_{j}(t), \quad t \in I_{T}, j=1, \ldots, r_{1},
$$

where $\Psi_{j} \in X^{*}=\mathscr{L}(X ; \mathbf{R})$ and $g_{j} \in C^{2+v_{j}}\left(I_{T} ; \mathbf{R}\right), v_{j} \in(0,1)$, $j=1, \ldots, r_{1}$. We will search for a solution vector $\left(\Theta, k_{1}, \ldots, k_{r_{1}}\right)$ of the inverse problem (234) and (235) such that $\Theta \in C^{1+\delta}\left(I_{T} ; \mathscr{D}(L)\right)$ and $k_{j} \in C^{\eta_{j}}\left(I_{T} ; \mathbf{R}\right), j=1, \ldots, r_{1}$, with the Hölder exponents $\delta$ and $\eta_{j}, j=1, \ldots, r_{1}$, to be made precise in the sequel. We stress that here we will not solve completely the mentioned inverse problem. For, its detailed treatment would lead us out of the aims of this paper. Our intention here is only to highlight how the main results of Section 5 allow to determine the correct functional framework in which the solution of the inverse problem has to be searched. However, a complete treatment of the inverse problem will be the object of a future paper.

Assuming that $\Theta \in C^{1+\delta}\left(I_{T} ; \mathscr{D}(L)\right)$ solves (234), we introduce the new unknown

$$
v(t, x)=D_{t} \Theta(t, x) \Longleftrightarrow \Theta(t, x)=\Theta_{0}(x)+\int_{0}^{t} v(s, x) \mathrm{d} s .
$$

Then, differentiating (234) with respect to time and using

$$
\begin{array}{rl}
D_{t} & \mathscr{K}\left(k_{i}, L_{i} \Theta\right)(t) \\
& =D_{t} \int_{0}^{t} k_{i}(t-s) L_{i} \Theta(s) \mathrm{d} s=D_{t} \int_{0}^{t} k_{i}(s) L_{i} \Theta(t-s) \mathrm{d} s \\
& =k_{i}(t) L_{i} \Theta(0)+\int_{0}^{t} k_{i}(s) L_{i} D_{t} \Theta(t-s) \mathrm{d} s \\
& =k_{i}(t) L_{i} \Theta_{0}+\int_{0}^{t} k_{i}(t-s) L_{i} v(s) \mathrm{d} s,
\end{array}
$$

we find that $v \in C^{\delta}\left(I_{T} ; \mathscr{D}(L)\right)$ solves the following degenerate integrodifferential problem:

$$
\begin{gathered}
D_{t}(M v(t)) \\
=\left[\lambda_{0} M+L\right] v(t)+\sum_{i=1}^{n_{1}}\left[\mathscr{K}\left(k_{i}, L_{i} v\right)(t)+k_{i}(t) y_{i}\right]+f(t), \\
M v(0)=M v_{0},
\end{gathered}
$$

where $y_{i}=L_{i} \Theta_{0}, i=1, \ldots, n_{1}, f=D_{t} \widetilde{g}$ and $M v_{0}=\left[\lambda_{0} M+\right.$ $L] \Theta_{0}+\widetilde{g}(0, \cdot)$ (indeed, since $M$ is the multiplication operator by the function $m_{1}$ independent of $t$, from the differential equation in (234) with $t=0$ we get $M v(0)=M D_{t} \Theta(0)=$ $\left.\left[\lambda_{0} M+L\right] \Theta(0)+\widetilde{g}(0)\right)$. Of course, $(238)$ is the special case $\left(i_{1}, i_{2}, n_{2}\right)=\left(i, i, n_{1}\right), h_{i}=k_{i}, i=1 \ldots, n_{1}$, of problem (160).

Conversely, assume that $v \in C^{\delta}\left(I_{T} ; \mathscr{D}(L)\right)$ solves (238). Then, the function $\Theta$ defined by (236) belongs to $C^{1+\delta}$ $\left(I_{T} ; \mathscr{D}(L)\right)$ and solves (234). Indeed, using the fact that $m_{1}$ 
does not depend on time and that $M, L$, and $L_{i}, i=1, \ldots, n_{1}$, are closed, we obtain

$$
\begin{aligned}
D_{t}( & M \Theta(t))-\left[\lambda_{0} M+L\right] \Theta(t) \\
& -\sum_{i=1}^{n_{1}} \mathscr{K}\left(k_{i}, L_{i} \Theta\right)(t)-\widetilde{g}(t) \\
= & D_{t}\left[M\left(\Theta_{0}+\int_{0}^{t} v(s) \mathrm{d} s\right)\right] \\
& -\left[\lambda_{0} M+L\right]\left[\Theta_{0}+\int_{0}^{t} v(s) \mathrm{d} s\right] \\
& -\sum_{i=1}^{n_{1}} \int_{0}^{t} k_{i}(t-s) L_{i}\left[\Theta_{0}+\int_{0}^{s} v(\xi) \mathrm{d} \xi\right] \mathrm{d} s \\
& -\widetilde{g}(0)-\int_{0}^{t} D_{s} \widetilde{g}(s) \mathrm{d} s \\
= & M v(t)-\left[\lambda_{0} M+L\right] \Theta_{0} \\
& -\int_{0}^{t}\left[\lambda_{0} M+L\right] v(s) \mathrm{d} s \\
& -\sum_{i=1}^{n_{1}} \int_{0}^{t} \int_{0}^{n_{1}} k_{i}(t-s) L_{i} \Theta_{0} \mathrm{~d} s \\
& -\int_{0}^{t} f(t-s)\left[\int_{0}^{s} L_{i} v(\xi) \mathrm{d} \xi\right] \mathrm{d} s \\
& -\int_{0} . \\
& \\
&
\end{aligned}
$$

Now, observe that

$$
\begin{aligned}
& M v(t)=M v_{0}+\int_{0}^{t} D_{s}(M v(s)) \mathrm{d} s \\
&=\left[\lambda_{0} M+L\right] \Theta_{0}+\widetilde{g}(0)+\int_{0}^{t} D_{s}(M v(s)) \mathrm{d} s, \\
& \int_{0}^{t} k_{i}(t-s) L_{i} \Theta_{0} \mathrm{~d} s=\int_{0}^{t} k_{i}(s) L_{i} \Theta_{0} \mathrm{~d} s=\int_{0}^{t} k_{i}(s) y_{i} \mathrm{~d} s, \\
& i=1, \ldots, n_{1},
\end{aligned}
$$

whereas an application of Fubini's theorem combined with the changes of variables $\xi=s-r, r-s=\tau$ and $t-s=\zeta$ easily yields for every $i=1, \ldots, n_{1}$ the following:

$$
\begin{aligned}
\int_{0}^{t} k_{i}( & t-s)\left[\int_{0}^{s} L_{i} v(\xi) \mathrm{d} \xi\right] \mathrm{d} s \\
= & \int_{0}^{t} k_{i}(t-s)\left[\int_{0}^{s} L_{i} v(s-r) \mathrm{d} r\right] \mathrm{d} s \\
= & \int_{0}^{t}\left[\int_{s}^{t} k_{i}(t-r) L_{i} v(r-s) \mathrm{d} r\right] \mathrm{d} s
\end{aligned}
$$

$$
\begin{aligned}
& =\int_{0}^{t}\left[\int_{0}^{t-s} k_{i}(t-s-\tau) L_{i} v(\tau) \mathrm{d} \tau\right] \mathrm{d} s \\
& =\int_{0}^{t} \mathscr{K}\left(k_{i}, L_{i} v\right)(t-s) \mathrm{d} s \\
& =\int_{0}^{t} \mathscr{K}\left(k_{i}, L_{i} v\right)(\zeta) \mathrm{d} \zeta=\int_{0}^{t} \mathscr{K}\left(k_{i}, L_{i} v\right)(s) \mathrm{d} s .
\end{aligned}
$$

Therefore, replacing (240)-(242) in (239), it follows for every $t \in I_{T}$ that

$$
\begin{aligned}
D_{t}(M \Theta(t))-\left[\lambda_{0} M+L\right] \Theta(t)-\sum_{i=1}^{n_{1}} \mathscr{K}\left(k_{i}, L_{i} \Theta\right)(t)-\tilde{g}(t) \\
=\int_{0}^{t}\left\{D_{s}(M v(s))-\left[\lambda_{0} M+L\right] v(s)\right. \\
\left.-\sum_{i=1}^{n_{1}}\left[\mathscr{K}\left(k_{i}, L_{i} v\right)(s)+k_{i}(s) y_{i}\right]-f(s)\right\} \mathrm{d} s,
\end{aligned}
$$

and the latter integral is equal to zero by virtue of (238). Since from (236) it follows that $\Theta(0)=\Theta_{0}$, we have thus shown that (234) and (238) are equivalent. Such an equivalence is the first step in solving the mentioned inverse problem of recovering the vector $\left(\Theta, k_{1}, \ldots, k_{r_{1}}\right)$ with the help of the additional information (235).

Let us now apply the linear functional $\Psi_{j}, j=1 \ldots, r_{1}$, to (238). Using

$$
\begin{aligned}
\Psi_{j}\left[D_{t}^{k}(M v(t))\right] & =\Psi_{j}\left[M D_{t}^{k+1} \Theta(t)\right]=D_{t}^{k+1} \Psi_{j}[M \Theta(t)] \\
& =D_{t}^{k+1} g_{j}(t), \quad k=0,1,
\end{aligned}
$$

we thus find the following system of $r_{1}$ equations for the $r_{1}$ unknown $k_{1}, \ldots, k_{r_{1}}$ :

$$
\begin{array}{r}
\sum_{i=1}^{r_{1}} \Psi_{j}\left[y_{i}\right] k_{i}(t) \\
=N_{j}(t)-\Psi_{j}[L v]-\sum_{i=1}^{n_{1}} \Psi_{j}\left[\mathscr{K}\left(k_{i}, L_{i} v\right)(t)\right], \\
j=1, \ldots, r_{1},
\end{array}
$$

where we have set (recall that $k_{r_{1}+n}=-D_{t} u_{n}, n=1,2$, are known)

$$
\begin{aligned}
N_{j}(t)= & \left(D_{t}-\lambda_{0}\right) D_{t} g_{j}(t)-\Psi_{j}[f(t)] \\
& -\sum_{n=1}^{2} \Psi_{j}\left[y_{r_{1}+n}\right] k_{r_{1}+n}(t), \quad j=1, \ldots, r_{1} .
\end{aligned}
$$


Therefore, if the matrix $\mathscr{U}:=\mathcal{U}_{\Psi_{1}, \ldots, \Psi_{r_{1}}}^{y_{1}, \ldots, y_{r_{1}}}=\left(\Psi_{i}\left[y_{j}\right]\right)_{i, j=1, \ldots, r_{1}}$ has determinant det $\mathscr{U} \neq 0$, then from Cramer's formula it follows that the solution $\left(k_{1}, \ldots, k_{r_{1}}\right)$ of $(245)$ is given by

$$
\begin{aligned}
k_{j}(t)=[\operatorname{det} \mathcal{U}]^{-1} \sum_{k=1}^{r_{1}}\left\{N_{k}(t)-\Psi_{k}[L v]\right. \\
\left.\quad-\sum_{i=1}^{n_{1}} \Psi_{k}\left[\mathscr{K}\left(k_{i}, L_{i} v\right)(t)\right]\right\} \mathscr{U}_{k, j} \\
=: \widetilde{R}_{j}\left(v, k_{1}, \ldots, k_{r_{1}}\right)(t), \quad j=1, \ldots, r_{1},
\end{aligned}
$$

with $\mathscr{U}_{k, j}, k, j=1, \ldots, r_{1}$, being the cofactor of the element $\Psi_{k}\left[y_{j}\right]$ of $\mathcal{U}$ (with the convention that $\mathscr{U}_{1,1}=1$ in the case of $r_{1}=1$ ). We have thus found a system of $r_{1}$ fixed-point equations for the $r_{1}$ unknown $k_{1}, \ldots, k_{r_{1}}$.

Now, let $Y_{\psi}^{r} \in\left\{(X, \mathscr{D}(A))_{\psi, r}, X_{A}^{\psi, r}\right\}, \psi \in(0,1), r \in[1, \infty]$, where $A$ is as in (233). Assume that $v_{0}$ in the initial condition $M v(0)=M v_{0}$ belongs to $\mathscr{D}(L)$ and that

$$
\begin{gathered}
k_{i} \in C^{\eta_{i}}\left(I_{T}, \mathbf{R}\right), \quad f \in C^{\mu}\left(I_{T} ; X\right), \quad \eta_{i}, \mu \in\left(\frac{1}{q^{\prime}}, 1\right), \\
i=1, \ldots, n_{1}, \\
y_{i} \in Y_{\gamma_{i}}^{p}, \quad v_{1}+f(0) \in Y_{\varphi}^{p}, \quad \gamma_{i}, \varphi \in\left(\frac{1}{q^{\prime}}, 1\right), \\
p \in[1, \infty], \quad i=1, \ldots, n_{1},
\end{gathered}
$$

where $v_{1}=\left(\lambda_{0} M+L\right) v_{0}$ and $q^{\prime}$ is the conjugate exponent of $q \in(1, \infty)$. Then (cf. (179) with $\left(i_{1}, i_{2}, n_{2}\right)=\left(i, i, n_{1}\right)$, $(\alpha, \beta, Z)=(1,1 / q, \mathbf{R})$, and $\left.h_{i}=k_{i}, i=1, \ldots, n_{1}\right)$, problem (238) is equivalent to the fixed-point equation

$$
\begin{aligned}
w(t) & =R\left(w, k_{1}, \ldots, k_{r_{1}}\right)(t)+\sum_{l=0}^{1} w_{l}\left(k_{1}, \ldots, k_{r_{1}}\right)(t) \\
& =: T\left(w, k_{1}, \ldots, k_{r_{1}}\right)(t),
\end{aligned}
$$

where $w=L\left(v-v_{0}\right)$ and

$$
\begin{gathered}
w_{0}\left(k_{1}, \ldots, k_{r_{1}}\right)=Q_{7} x_{0}+\sum_{i=1}^{n_{1}}\left[Q_{6}\left(k_{i}, L_{i} v_{0}\right)+Q_{5} \tilde{k}_{i}\right]+Q_{5} \tilde{f}, \\
w_{1}\left(k_{1}, \ldots, k_{r_{1}}\right)=-\sum_{i=1}^{n_{1}}\left[Q_{3}\left(k_{i}, L_{i} v_{0}\right)+Q_{4}\left(k_{i}, y_{i}\right)\right]-Q_{2} f \\
R\left(w, k_{1}, \ldots, k_{r_{1}}\right)=\lambda_{0}\left[Q_{5}\left(A^{-1} w\right)-Q_{2}\left(A^{-1} w\right)\right] \\
+\sum_{i=1}^{n_{1}}\left[Q_{6}\left(k_{i}, S_{i} w\right)-Q_{3}\left(k_{i}, S_{i} w\right)\right] .
\end{gathered}
$$

Here, the $Q_{j}$ 's, $j=2, \ldots, 6$, are defined by (106)-(110), $S_{i}=$ $L_{i} L^{-1}$, and the functions $\tilde{f}, \tilde{k}_{i}$ and $Q_{7} x_{0}$ are defined by $\tilde{f}(t)=$ $f(t)-f(0), \tilde{k}_{i}(t)=\left[k_{i}(t)-k_{i}(0)\right] y_{i}$, and $\left[Q_{7} x_{0}\right](t)=\left(\mathrm{e}^{t A}-\right.$ I) $x_{0}$, respectively, where (cf. (174)) $x_{0}=\sum_{i=1}^{n_{1}} k_{i}(0) y_{i}+v_{1}+$ $f(0)$.

Then, since $v=L^{-1} w+v_{0}$, if we set $R_{j}\left(w, k_{1}, \ldots, k_{r_{1}}\right)=$ $\widetilde{R}_{j}\left(L^{-1} w+v_{0}, k_{1}, \ldots, k_{r_{1}}\right), j=1, \ldots, r_{1}$, and

$$
\begin{aligned}
& \Xi\left(w, k_{1}, \ldots, k_{r_{1}}\right) \\
& =\left(T\left(w, k_{1}, \ldots, k_{r_{1}}\right), R_{1}\left(w, k_{1}, \ldots, k_{r_{1}}\right),\right. \\
& \left.\ldots, R_{r_{i}}\left(w, k_{1}, \ldots, k_{r_{1}}\right)\right),
\end{aligned}
$$

from (247) and (249) we deduce that to solve the inverse problems (234) and (235) for the unknown vector $\left(\Theta, k_{1}, \ldots, k_{r_{1}}\right)$, it suffices to show that the fixed-point equation

$$
\left(w, k_{1}, \ldots, k_{r_{1}}\right)=\Xi\left(w, k_{1}, \ldots, k_{r_{1}}\right)
$$

has a unique solution. In general, this will be done by proving that $\Xi$ is a contraction map in the Banach space

$$
\begin{gathered}
Z_{\delta, \eta_{1}, \ldots, \eta_{r_{1}}}=C^{\delta}\left(I_{T} ; X\right) \times C^{\eta_{1}}\left(I_{T} ; \mathbf{R}\right) \times \cdots \times C^{\eta_{r_{1}}}\left(I_{T} ; \mathbf{R}\right), \\
\left\|\left(f_{0}, f_{1}, \ldots, f_{r_{1}}\right)\right\|_{Z_{\delta, \eta_{1}, \ldots, \eta_{r_{1}}}} \\
=\left\|f_{0}\right\|_{\delta, 0, T ; X}+\left\|f_{1}\right\|_{\eta_{1}, 0, T ; \mathbf{R}}+\cdots+\left\|f_{r_{1}}\right\|_{\eta_{r_{1}, 0, T ; \mathbf{R}^{\prime}}}
\end{gathered}
$$

at least for opportunely chosen Hölder exponents $\delta \in(0,1)$ and $\eta_{i} \in\left(1 / q^{\prime}, 1\right), i=1, \ldots, r_{1}$, and, eventually, sufficiently small values of $T>0$. It is just in the choice of $\delta$ and the $\eta_{i}$ 's that the main result of Section 5 plays a key role. The Hölder exponents have to be chosen so that the direct problem (234) in which the $k_{i}$ 's are assumed to be known is well posed. Due to the shown equivalence between problems (234) and (238), the well-posedness of the direct problem (234) is then a consequence of Theorem 48 and formula (236). More precisely, recalling Remark 50 for the case $\alpha=1$, an application of that theorem yields the following maximal time regularity result for the solution $\Theta$ of (234).

Theorem 57. Let $X, \mathscr{D}(M), \mathscr{D}(L)$, and $\mathscr{D}\left(L_{i}\right), i=1, \ldots, n_{1}$, $n_{1}=r_{1}+2, r_{1} \in \mathbf{N}$, be defined by (225) and (226) with $q \in$ $(1,2)$. Let $M, L$, and $L_{i}, i=1, \ldots, n_{1}$, be defined by (227)(229) through (209), (212), and (215)-(221), and let (230) and (231) be satisfied. Further, let $(A, \mathscr{D}(A))$ be defined by (233), and let $Y_{\psi}^{r} \in\left\{(X, \mathscr{D}(A))_{\psi, r}, X_{A}^{\psi, r}\right\}, \psi \in(0,1), r \in[1, \infty]$. Let $\eta_{i} \in\left(1 / q^{\prime}, 1\right)$ and $\gamma_{i}, \varphi \in\left(2 / q^{\prime}, 1\right), i=1, \ldots, n_{1}$, and assume that

$$
\begin{array}{r}
k_{i} \in C^{\eta_{i}}\left(I_{T} ; \mathbf{R}\right), \quad i=1, \ldots, n_{1}, \quad \Theta_{0} \in \mathscr{D}(L), \\
\left(\lambda_{0} M+L\right) \Theta_{0}+\widetilde{g}(0, \cdot)=M v_{0} \text { for some } v_{0} \in \mathscr{D}(L), \\
L_{i} \Theta_{0} \in Y_{\gamma_{i}}^{r}, \quad v_{1}+D_{t} \tilde{g}(0, \cdot) \in Y_{\varphi}^{r}, \quad i=1, \ldots, n_{1}, \\
r \in[1, \infty],
\end{array}
$$


where $k_{i}, i=1, \ldots, n_{1}, \tilde{g}$ and $\lambda_{0}$ are defined by (222) and (223) through (211) and (216), whereas $v_{1}=\left(\lambda_{0} M+L\right) v_{0}$. Let $\gamma=$ $\min _{i=1, \ldots, n_{1}}\left\{\gamma_{i}, \varphi\right\}$ and $\tau=\min _{i=1, \ldots, n_{1}}\left\{\eta_{i}, \gamma-1 / q^{\prime}\right\}$, and let $I_{1,1 / q, \tau} \subseteq\left(1 / q^{\prime}, 1 / 2\right)$ be the interval defined by (cf. (207) with $\beta=1 / q)$

$$
\begin{aligned}
& I_{1,1 / q, \tau}=\left(\frac{1}{q^{\prime}}, \tau\right], \quad \text { if } \tau \in\left(\frac{1}{q^{\prime}}, \frac{1}{2}\right), \\
& I_{1,1 / q, \tau}=\left(\frac{1}{q^{\prime}}, \frac{1}{2}\right), \quad \text { if } \tau \in\left[\frac{1}{2}, 1\right) .
\end{aligned}
$$

Then, for every fixed $\delta \in I_{1,1 / q, \tau}$ problem (234), or, equivalently, problem (224), admits a unique strict solution $\Theta \in C^{1+\delta}$ $\left(I_{T} ; \mathscr{D}(L)\right)$ satisfying $D_{t} \Theta(0)=v_{0}$ and such that $D_{t} M \Theta, L \Theta \in$ $C^{1+\delta}\left(I_{T} ; X\right)$, provided that $D_{t} \widetilde{g} \in C^{\mu}\left(I_{T} ; X\right), \mu \in\left[\delta+1 / q^{\prime}, 1\right)$.

Proof. Apply Theorem 48 with $\left(i_{1}, i_{2}, n_{2}\right)=\left(i, i, n_{1}\right),(\alpha, \beta$, $Z)=(1,1 / q, \mathbf{R})$, and $h_{i}=k_{i}, i=1, \ldots, n_{1}$, to the equivalent problem (238). Since $M$ is the multiplication operator by the function $m_{1}$ independent of $t$, the assertion then follows from $D_{t} \Theta=v \in C^{\delta}\left(I_{T} ; \mathscr{D}(L)\right), D_{t} \Theta(0)=v(0), D_{t} L \Theta=L v \in$ $C^{\delta}\left(I_{T} ; X\right)$ and $D_{t}^{2} M \Theta=D_{t} M v \in C^{\delta}\left(I_{T} ; X\right)$.

Larger values of $q$ in Theorem 57 can be obtained assuming more smoothness and some order of vanishing for the function $m_{1}$. In fact, let $m_{1} \in C^{1}(\bar{\Omega})$ be such that the following estimate holds for some positive constant $K$ :

$$
\begin{array}{r}
\left|\nabla m_{1}(x)\right|:=\left\{\sum_{j=1}^{N}\left[D_{x_{j}} m_{1}(x)\right]^{2}\right\}^{1 / 2} \leq K\left[m_{1}(x)\right]^{\vartheta}, \\
x \in \bar{\Omega}, \quad \vartheta \in(0,1) .
\end{array}
$$

Then (232) holds with $\beta=1 / q$ being replaced by (cf. [41, formulae (3.23) and (4.41)]):

$$
\begin{aligned}
& \beta=\frac{1}{2-\vartheta}, \quad \text { if } q \in(2-\vartheta, 2), \\
& \beta=\frac{2}{q(2-\vartheta)}, \quad \text { if } q \in[2, \infty) .
\end{aligned}
$$

(precisely, in [41, formula (3.23)] it is shown that $(|\lambda|+$ 1) $\|M u\|_{q ; \Omega}^{q(2-9) / 2} \leq C_{q}\left[\|f\|_{q ; \Omega}\|M u\|_{q ; \Omega}^{-1+q(2-9) / 2}+\|f\|_{q ; \Omega}^{q(2-9) / 2}\right]$, where $u=(\lambda M-L)^{-1} f$ and $q \in[2, \infty)$. Using (cf. [41, formula (2.15)]) $\|M u\|_{q ; \Omega} \leq\left\|m_{1}\right\|_{\infty ; \Omega}\|u\|_{q ; \Omega} \leq C\left\|m_{1}\right\|_{\infty ; \Omega}$ $\|f\|_{q ; \Omega}$, we thus find that $(|\lambda|+1)\|M u\|_{q ; \Omega}^{q(2-9) / 2} \leq C_{q}[(C$ $\left.\left.\left\|m_{1}\right\|_{\infty ; \Omega}\right)^{-1+q(2-9) / 2}+1\right]\|f\|_{q ; \Omega}^{q(2-9) / 2}$; that is, $\| M(\lambda M-$ $L)^{-1} \|_{\mathscr{L}(X)} \leq\left\{C_{q}\left[\left(C\left\|m_{1}\right\|_{\infty ; \Omega}\right)^{-1+q(2-9) / 2}+1\right]\right\}^{2 /[q(2-9)]}(|\lambda|+$ $\left.1)^{-2 /[q(2-9)]}\right)$. Under (256) we thus find the following better result, where $q$ may be greater than two.

Theorem 58. Let (256) holds, and let $X,(M, \mathscr{D}(M)),(L, \mathscr{D}$ $(L)),\left(L_{i}, \mathscr{D}\left(L_{i}\right)\right), i=1, \ldots, n_{1}$, be as in Theorem 57, but with $q \in(2-9,2) \cup[2,4 /(2-9))$. Let (254) be fulfilled, but with $\eta_{i} \in(1-\beta, 1)$ and $\gamma_{i}, \varphi \in(2-2 \beta, 1), i=1, \ldots, n_{1}$, where $\beta$ is as in (257). Let $\gamma=\min _{i=1, \ldots, n_{1}}\left\{\gamma_{i}, \varphi\right\}$ and $\tau=\min _{i=1, \ldots, n_{1}}\left\{\eta_{i}, \beta+\right.$ $\gamma-1\}$, and let $I_{1, \beta, \tau}$ be as in (207). Then, for every fixed $\delta \in I_{1, \beta, \tau}$ problem (234), or, equivalently, problem (224), admits a unique strict solution $\Theta \in C^{1+\delta}\left(I_{T} ; \mathscr{D}(L)\right)$ satisfying $D_{t} \Theta(0)=v_{0}$ and such that $D_{t} M \Theta, L \Theta \in C^{1+\delta}\left(I_{T} ; X\right)$, provided that $D_{t} \tilde{g} \in$ $C^{\mu}\left(I_{T} ; X\right), \mu \in[\delta+1-\beta, 1)$.

Proof. It suffices to observe that for every $\vartheta \in(0,1)$ and $q \epsilon$ $(2-\vartheta, 2) \cup[2,4 /(2-\vartheta))$, the number $\beta$ in $(257)$ satisfies $\beta>$ $1 / 2$. Hence, proceeding as in the proofs of Theorem 57 , except for replacing there $\beta=1 / q$ with $\beta$ as in (257), we get the assertion.

\section{Appendix}

Here we clarify why the definition of $Q_{2}$ in [20] has to be modified in accordance to that in this paper. To avoid confusion with the present notation, we will denote the operator $Q_{2}$ in [20] with $S_{2}$. Precisely, in [20, formula (4.12)], $S_{2}$ was defined as follows:

$$
\left[S_{2} g_{2}\right](t):=\int_{0}^{t}\left[(-A)^{1}\right]^{\circ} \mathrm{e}^{(t-s) A} g_{2}(s) \mathrm{d} s, \quad t \in[0, T]
$$

and considered as acting on functions $g_{2} \in C_{0}^{\delta_{2}}([0, T] ; X)$, $\delta_{2} \in((3-2 \alpha-\beta) / \alpha, 1), 3 \alpha+\beta>3$. Even though $g_{2}(0)=0$, formula (A.1) may have no sense, since

$$
\left\|S_{2} g_{2}(t)\right\|_{X} \leq \widetilde{c}_{\alpha, \beta, 1}\left|g_{2}\right|_{\delta_{2}, 0, t ; X} \int_{0}^{t}(t-s)^{(\beta-2) / \alpha} s^{\delta_{2}} \mathrm{~d} s,
$$

and the integral on the right is not convergent, the exponent $(\beta-2) / \alpha$ being less or equal than -1 . It is for this reason that $g_{2}(s)$ in (A.1) has to be replaced with the increment $g_{2}(s)-$ $g_{2}(t)$ as in formula (106) (see inequality (118)) and to introduce the operator $Q_{5}$ as in (109). Of course, as a consequence, the definitions of $Q_{3}$ and $Q_{4}$ in [20, Lemmas 4.6 and 4.8] as $S_{2} \mathscr{K}\left(g_{3_{1}}, g_{3_{2}}\right)$ and $S_{2}\left(g_{4} y\right)$, respectively, have to be changed too in accordance with the present formulae (107) and (108) containing the increments $\mathscr{K}\left(g_{3_{1}}, g_{3_{2}}\right)(s)-\mathscr{K}\left(g_{3_{1}}, g_{3_{2}}\right)(t)$ and $\left[g_{4}(s)-g_{4}(t)\right] y$. To this purpose, we want to make clear that, contrarily to [20, Lemma 4.4], the statement and the proof of [20, Lemma 4.8] is correct, since there the function inside the integral on the right-hand side of (A.1) takes its values in an opportune intermediate space $X_{A}^{\theta, r}$. However, the correctness of that lemma does not suffice to proceed as in [20, Section 5] to solve problem (160) with $n_{1}=n_{2}=1$.

For the reader's convenience we thus now indicate how to change the definitions of the functions $w_{j}, j=0,1$, and the operator $R w$ in [20, formulae (5.8)-(5.10)], and we state the amended version of [20, Theorems 5.6 and 5.7]. First, according to [20] where only this case was treated, let $n_{1}=$ $n_{2}=1$ in problem (160), and write $k, h, y$ in place of $k_{1}, h_{1}$ and $y_{1}$, respectively. Then, under the same assumptions on the vector $(\alpha, \beta, k, h, f)$ as those in the present Section 5 , it can 
be shown that problem (160) with $n_{1}=n_{2}=1$ is equivalent to the fixed-point equation (179), where (cf. (180)-(182))

$$
\begin{gathered}
w_{0}=Q_{7} x_{0}+Q_{6}\left(k, L_{1} v_{0}\right)+Q_{5} \tilde{h}+Q_{5} \tilde{f} \\
w_{1}=-Q_{3}\left(k, L_{1} v_{0}\right)-Q_{4}(h, y)-Q_{2} f \\
R w:=\lambda_{0}\left[Q_{5}\left(A^{-1} w\right)-Q_{2}\left(A^{-1} w\right)\right] \\
+Q_{6}(k, S w)-Q_{3}(k, S w) .
\end{gathered}
$$

Here, $x_{0}=v_{1}+h(0) y+f(0), v_{1}=\left(\lambda_{0} M+L\right) v_{0}$, is the value at $t=0$ of the function $F_{w}$ defined by (169) with $n_{l}=n_{2}=1$, $Q_{7} x_{0}, \tilde{f}$ and $\tilde{h}$ are defined, respectively, by $\left(\mathrm{e}^{t A}-I\right) x_{0}, f(t)-$ $f(0)$ and $[h(t)-h(0)] y, S$ is the operator $L_{1} L^{-1} \in \mathscr{L}(X)$, and the $Q_{j}$ 's, $j=2, \ldots, 6$, are as in (106)-(110). Formulae (A.3) replace the definitions of $w_{0}, w_{1}$ and $R w$ in [20, formulae (5.8)-(5.10)]. Therefore, from Lemmas 42, 45, and 46 and Corollary 44 with $n_{1}=n_{2}=1$ we obtain the following version of Theorem 48.

Theorem A.1. Assume (161) and $v_{0} \in \mathscr{D}(L)$, and let $5 \alpha+2 \beta>$ 6 in (H2). Assume that $k \in C^{\eta}\left(I_{T} ; Z\right), h \in C^{\sigma}\left(I_{T} ; \mathbf{C}\right), y \in Y_{\theta}^{r}$, and $\left(\lambda_{0} M+L\right) v_{0}+f(0) \in Y_{\varphi}^{r}$, where $\eta, \sigma \in((3-2 \alpha-\beta) / \alpha, 1)$, $\theta, \varphi \in(5-3 \alpha-2 \beta, 1)$, and $r \in[1, \infty]$. Let $\gamma=\min \{\theta, \varphi\}$ and $\tau=\min \{\eta, \sigma,(\alpha+\beta+\gamma-2) / \alpha\}$. Then, for every fixed $\delta \in I_{\alpha, \beta, \tau}$ the problem

$$
\begin{gathered}
D_{t}(M v(t))=\left[\lambda_{0} M+L\right] v(t)+\mathscr{K}\left(k, L_{1} v\right)(t) \\
+h(t) y+f(t), \quad t \in I_{T}, \\
M v(0)=M v_{0}
\end{gathered}
$$

admits a unique strict solution $v \in C^{\delta}\left(I_{T} ; \mathscr{D}(L)\right)$ satisfying $v(0)=v_{0}$ and such that $L v, D_{t} M v \in C^{\delta}\left(I_{T} ; X\right)$, provided that $f \in C^{\mu}\left(I_{T} ; X\right), \mu \in[\delta+(3-2 \alpha-\beta) / \alpha, 1)$.

Theorem A.1 substitutes [20, Theorem 5.6 and 5.7]. Notice that, differently than [20], here only one statement occurs. In fact, the more suitable procedure followed in this paper makes the separation in [20] of two distinct intervals in which $\gamma$ may vary totally unneeded. Finally, letting $n_{1}=n_{2}=1$ in Theorems 52, 5.14, 54, and 56, we obtain the correct versions of [20, Theorems 5.11, 53, and 5.16] for the subcases of (A.4) corresponding to the choices $\lambda_{0}=h=0, \lambda_{0}=f=0$, $\lambda_{0}=k=h=0$, and $\lambda_{0}=k=f=0$, respectively. For saving space, we leave this easy task to the reader.

\section{References}

[1] A. Favini and A. Yagi, "Multivalued linear operators and degenerate evolution equations," Annali di Matematica Pura ed Applicata, vol. 163, pp. 353-384, 1993.

[2] A. Favini and A. Yagi, Degenerate Differential Equations in Banach Spaces, vol. 215, Marcel Dekker, New York, NY, USA, 1999.

[3] A. Yagi, "Generation theorem of semigroup for multivalued linear operators," Osaka Journal of Mathematics, vol. 28, no. 2, pp. 385-410, 1991.
[4] P. Acquistapace and B. Terreni, "Existence and sharp regularity results for linear parabolic nonautonomous integro-differential equations," Israel Journal of Mathematics, vol. 53, no. 3, pp. 257303, 1986.

[5] G. Da Prato, M. Iannelli, and E. Sinestrari, "Regularity of solutions of a class of linear integro-differential equations in Banach spaces," Journal of Integral Equations, vol. 8, no. 1, pp. 27-40, 1985.

[6] G. Da Prato and M. Iannelli, "Existence and regularity for a class of integro-differential equations of parabolic type," Journal of Mathematical Analysis and Applications, vol. 112, no. 1, pp. 3655, 1985.

[7] G. Da Prato, "Abstract differential equations, maximal regularity and linearization," in Proceedings of the Symposia in Pure Mathematics, vol. 45, part 1, pp. 359-370, 1986.

[8] A. Lunardi and E. Sinestrari, " $C^{\alpha}$-regularity for nonautonomous linear integro-differential equations of parabolic type," Journal of Differential Equations, vol. 63, no. 1, pp. 88-116, 1986.

[9] A. Lunardi, Analytic Semigroups and Optimal Regularity in Parabolic Problems, Birkhäuser, Basel, Switzerland, 1995.

[10] J. Prüss, "On linear Volterra equations of parabolic type in Banach spaces," Transactions of the American Mathematical Society, vol. 301, no. 2, pp. 691-721, 1987.

[11] E. Sinestrari, "On the abstract Cauchy problem of parabolic type in spaces of continuous functions," Journal of Mathematical Analysis and Applications, vol. 107, no. 1, pp. 16-66, 1985.

[12] E. Hille and R. S. Phillips, Functional Analysis and Semi-Groups, American Mathematical Society, Providence, RI, USA, 1957.

[13] C. Wild, "Semi-groupes de croissance $\alpha<1$ holomorphes," Comptes Rendus de l'Académie des Sciences, vol. 285, no. 6, pp. A437-A440, 1977 (French).

[14] K. Taira, "On a degenerate oblique derivative problem with interior boundary conditions," Proceedings of the Japan Academy, vol. 52, no. 9, pp. 484-487, 1976.

[15] K. Taira, "The theory of semigroups with weak singularity and its applications to partial differential equations," Tsukuba Journal of Mathematics, vol. 13, no. 2, pp. 513-562, 1989.

[16] W. von Wahl, "Gebrochene Potenzen eines elliptischen Operators und parabolische Differentialgleichungen in Räumen Höldersteiger Funktionen," Nachrichten der Akademie der Wissenschaften in Göttingen. II, vol. 11, pp. 231-258, 1972 (German).

[17] W. von Wahl, "Lineare und semilineare parabolische Differentialgleichungen in Räumen hölderstetiger Funktionen," Abhandlungen aus dem Mathematischen Seminar der Universität Hamburg, vol. 43, pp. 234-262, 1975 (German).

[18] W. von Wahl, "Neue Resolventenabschätzungen für elliptische Differentialoperatoren und semilineare parabolische Gleichungen," Abhandlungen aus dem Mathematischen Seminar der Universität Hamburg, vol. 46, pp. 179-204, 1977 (German).

[19] A. Favaron, "Optimal time and space regularity for solutions of degenerate differential equations," Central European Journal of Mathematics, vol. 7, no. 2, pp. 249-271, 2009.

[20] A. Favaron and A. Favini, "Maximal time regularity for degenerate evolution integro-differential equations," Journal of Evolution Equations, vol. 10, no. 2, pp. 377-412, 2010.

[21] A. Favini and A. Yagi, "Space and time regularity for degenerate evolution equations," Journal of the Mathematical Society of Japan, vol. 44, no. 2, pp. 331-350, 1992.

[22] A. Favini, A. Lorenzi, and H. Tanabe, "Singular integrodifferential equations of parabolic type," Advances in Differential Equations, vol. 7, no. 7, pp. 769-798, 2002. 
[23] A. Favini, A. Lorenzi, and H. Tanabe, "Singular evolution integro-differential equations with kernels defined on bounded intervals," Applicable Analysis, vol. 84, no. 5, pp. 463-497, 2005.

[24] A. Favaron and A. Favini, "Fractional powers and interpolation theory for multivalued linear operators and applications to degenerate differential equations," Tsukuba Journal of Mathematics, vol. 35, no. 2, pp. 259-323, 2011.

[25] R. Cross, Multivalued Linear Operators, vol. 213, Marcel Dekker, New York, NY, USA, 1998.

[26] A. Favini and A. Yagi, "Quasilinear degenerate evolution equations in Banach spaces," Journal of Evolution Equations, vol. 4, no. 3, pp. 421-449, 2004.

[27] H. Triebel, Interpolation Theory, Function Spaces, Differential Operators, vol. 18, North-Holland Publishing, Amsterdam, The Netherlands, 1978.

[28] H. Berens, Interpolationsmethoden zur Behandlung von Approximationsprozessen auf Banachräumen, Lecture Notes in Mathematics, Springer, Berlin, Germany, 1968 (German).

[29] E. Gagliardo, "Interpolation d'espaces de Banach et applications. III," Comptes Rendus de l'Académie des Sciences, vol. 248, pp. 3517-3518, 1959 (French).

[30] P. Grisvard, "Commutativité de deux foncteurs d'interpolation et applications," Journal de Mathématiques Pures et Appliquées, vol. 45, pp. 143-206, 1966 (French).

[31] H. Berens and P. L. Butzer, "Approximation theorems for semi-group operators in intermediate spaces," Bulletin of the American Mathematical Society, vol. 70, pp. 689-692, 1964.

[32] A. Belleni-Morante, "An integro-differential equation arising from the theory of heat conduction in rigid materials with memory," Unione Matematica Italiana, vol. 15, no. 2, pp. 470482, 1978.

[33] G. Da Prato and M. Iannelli, "Linear integro-differential equations in Banach spaces," Rendiconti del Seminario Matematico dell'Università di Padova, vol. 62, pp. 207-219, 1980.

[34] G. Da Prato, M. Iannelli, and E. Sinestrari, "Temporal regularity for a class of integro-differential equations in Banach spaces," Bollettino Unione Matematica Italiana, vol. 2, no. 1, pp. 171-185, 1983.

[35] A. Favini, A. Lorenzi, and H. Tanabe, "Degenerate integrodifferential equations of parabolic type with Robin boundary conditions: $L^{2}$-theory," Journal of the Mathematical Society of Japan, vol. 61, no. 1, pp. 133-176, 2009.

[36] D. Gilbarg and N. S. Trudinger, Elliptic Partial Differential Equations of Second Order, Springer, Berlin, Germany, 2001, Reprint of the 1998 edition.

[37] B. D. Coleman and M. E. Gurtin, "Equipresence and constitutive equations for rigid heat conductors," Zeitschrift für Angewandte Mathematik und Physik, vol. 18, pp. 199-208, 1967.

[38] M. E. Gurtin, "On the thermodynamics of materials with memory," Archive for Rational Mechanics and Analysis, vol. 28, no. 1, pp. 40-50, 1968.

[39] R. K. Miller, "An integro-differential equation for rigid heat conductors with memory," Journal of Mathematical Analysis and Applications, vol. 66, no. 2, pp. 313-332, 1978.

[40] J. W. Nunziato, "On heat conduction in materials with memory," Quarterly of Applied Mathematics, vol. 29, pp. 187-204, 1971.

[41] A. Favini, A. Lorenzi, H. Tanabe, and A. Yagi, "An $L^{p}$-approach to singular linear parabolic equations in bounded domains," Osaka Journal of Mathematics, vol. 42, no. 2, pp. 385-406, 2005. 


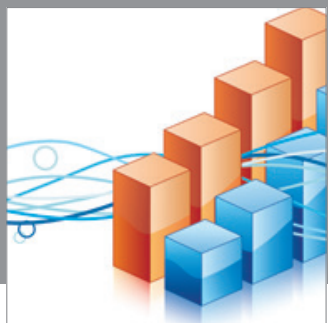

Advances in

Operations Research

mansans

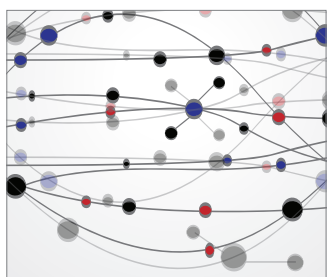

The Scientific World Journal
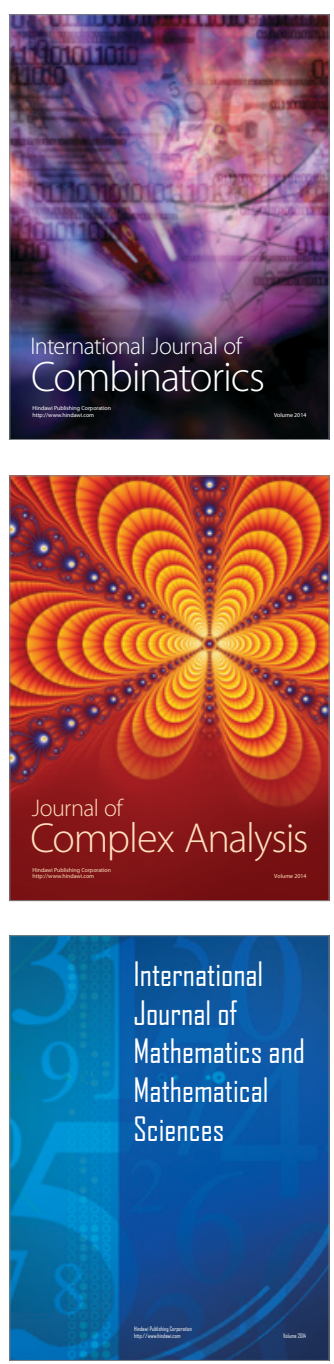
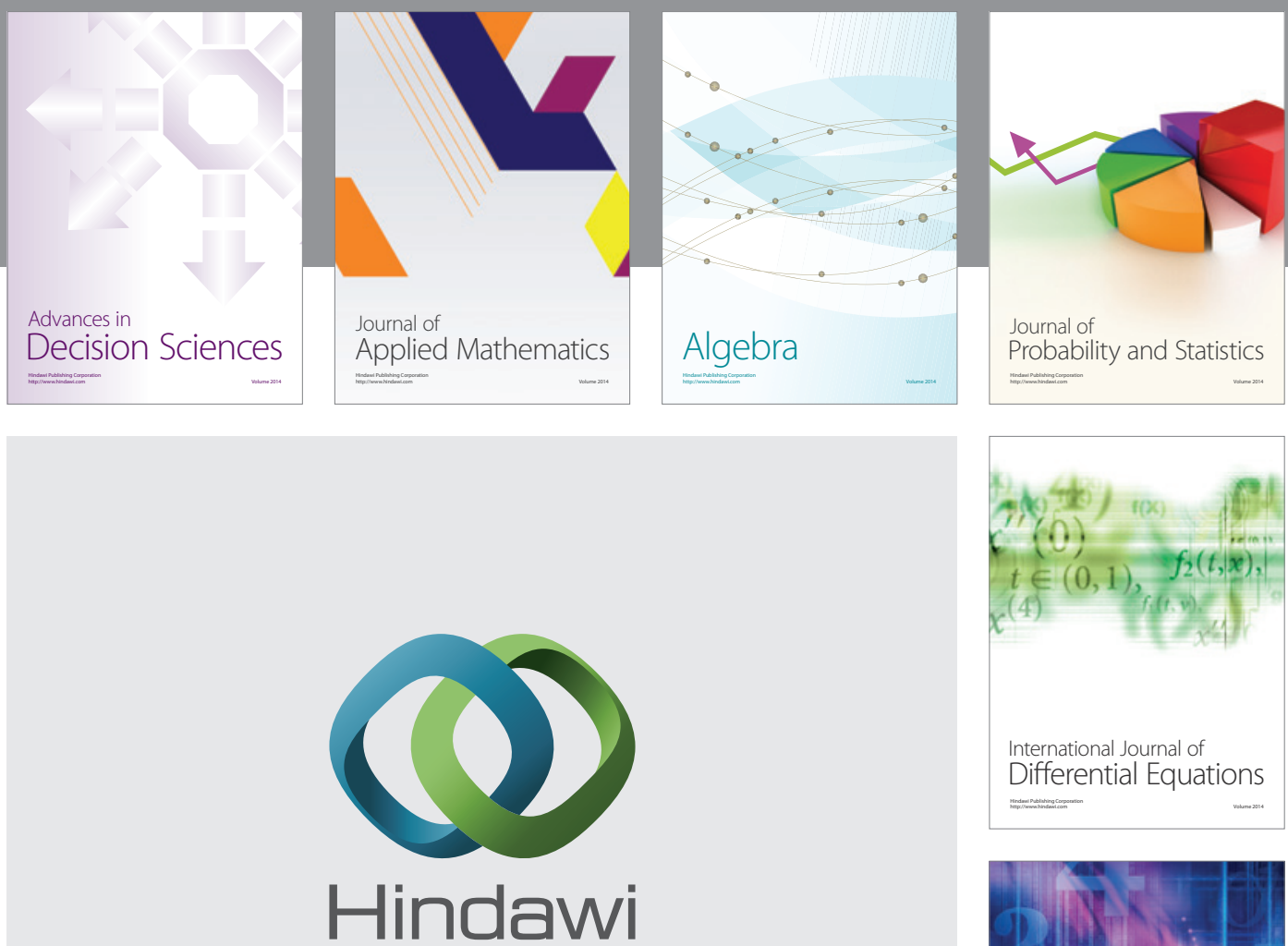

Submit your manuscripts at http://www.hindawi.com
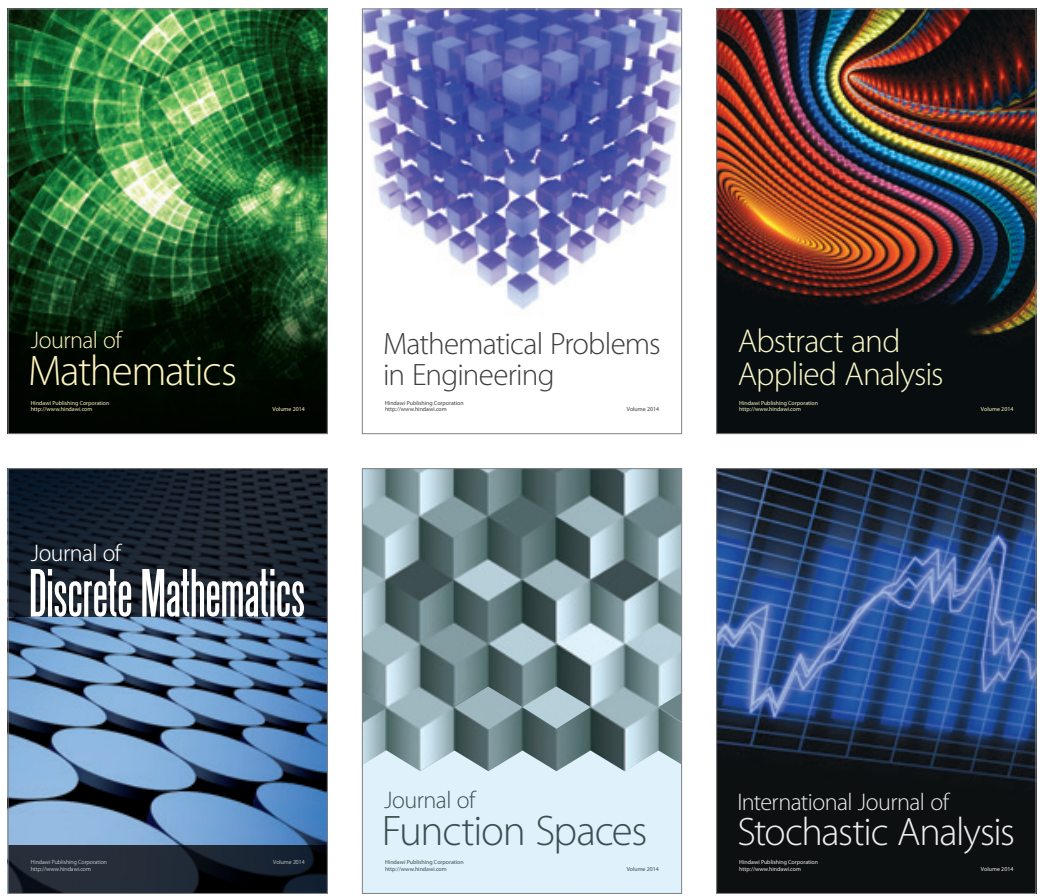

Journal of

Function Spaces

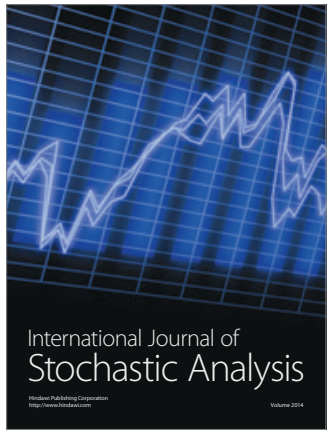

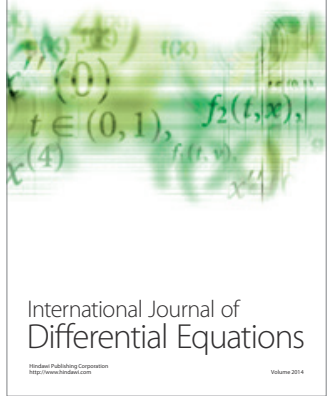
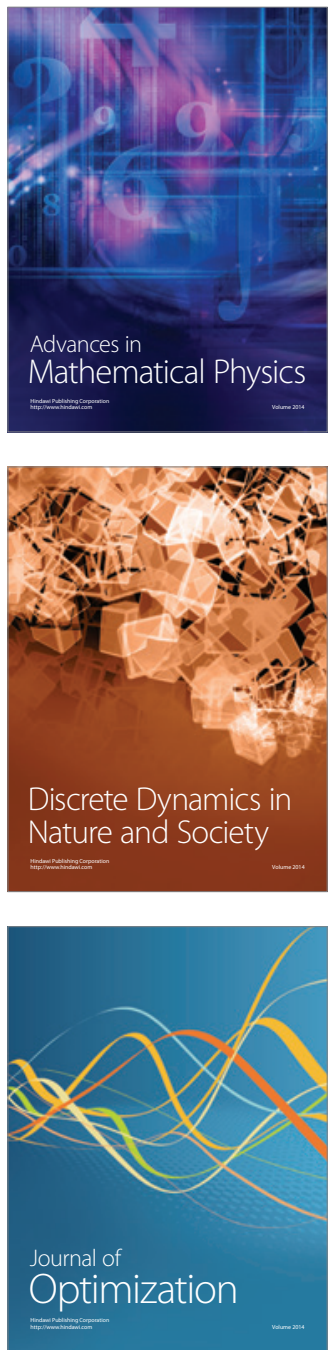